\title{
MSU-DOE \\ Plant Research Laboratory
}

\section{Twenty-fifth Annual Report 1990}

$$
\text { ACO2-76ER O1338 }
$$

\section{DISCLAIMER}

This report was prepared as an account of work sponsored by an agency of the United States Government. Neither the United States Government nor any agency thereof, nor any of their employees, makes any warranty, express or implied, or assumes any legal liability or responsibility for the accuracy, completeness, or usefulness of any information, apparatus, product, or process disclosed, or represents that its use would not infringe privately owned rights. Reference herein to any specific commercial product, process, or service by trade name, trademaik. manufacturer, or otherwise does not necessarily constitute or imply its endorsement, recommendation, or favoring by the United States Government or any agency thereof. The views and opinions of authors expressed herein do not necessarily state or reflect those of the United States Government or any agency thereof.

\section{Michigan State University East Lansing, Michigan 48824, USA (517) 353-2270}

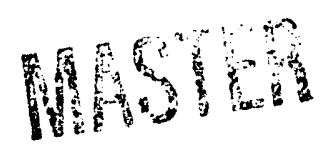




\section{CONTENTS}

Director's Introduction $\ldots \ldots \ldots \ldots \ldots \ldots \ldots \ldots \ldots \ldots \ldots \ldots \ldots$

Research Reports . ...................... 4

Molecular Basis of Symbiotic Plant-Microbe Interactions,

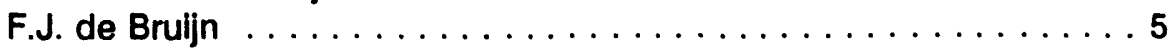

Enzymatic Mechanisms and Regulation of Plant Cell Wall Biosynthesis,

D.P. Delmer . . . . . . . . . . . . . . . . . . . . 13

Molecular Mechanisms that Regulate the Expression of Genes in Plants,

P.J. Green .............................. 19

Resistance of Plants to Environmental Stress,

A. Hanson . . . . . . . . . . . . . . . . . . . . . 23

Studies on Hormone Biosynthesis and Action,

H. Kende .............................. 28

Plant Cell Wail Proteins,

D.T.A. Lamport . . . . . . . . . . . . . . . . . . . . 37

Interaction of Nuclear and Organelle Genomes,

L. McIntosh . . . . . . . . . . . . . . . . . . . . . . 45

Sensory Transduction in Plants,

K. Poff ............................. 54

Molecular Mechanisms of Trafficking in the Plant Cell,

N. Raikhel ...............................6.62

Regulation of Lipid Metabolism,

C. Somerville ............................ 67

Molecular Bases of Plant Disease Resistance Mechanisms,

S. Somerville . . . . . . . . . . . . . . . . . . . . . . . . . 75

Biochemical and Molecular Aspects of Plant Pathogenesis,

J.D. Walton . . . . . . . . . . . . . . . . . . . . . . 80

Developmental Biology of Nitrogen-fixing Cyanobacteria,

C.P. Wolk . . . . . . . . . . . . . . . . . . . . . . . 87

Environmental Control of Plant Development and its Relation

to Plant Hormones,

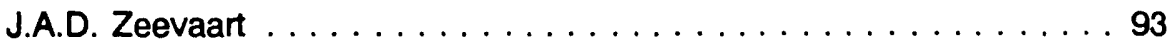

Personnel . . . . . . . . . . . . . . . . . . . . . . . . . . . . . 101

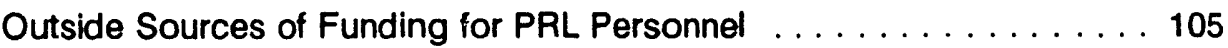

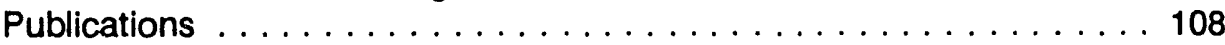

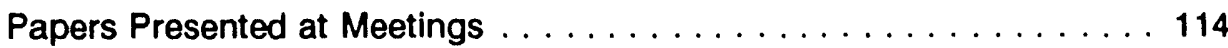

Seminar Schedule . . . . . . . . . . . . . . . . . . . . . . 119

$25^{\text {th }}$ Anniversary Symposium . . . . . . . . . . . . . . 123 


\section{DIRECTOR'S INTRODUCTION}

1990 saw the 25th anniversary of the Piant Research Laboratory (PRL) as a center for research and training in experimental plant biology. We celebrated this event with an excellent symposium, the arrival of a new research group, and continued intensive pursuit of our scientific goals. During the past twenty-five years, over four hundred graduate students and postdoctoral research associates have been trained at the PRL. Many of these have gone on to positions of considerable eminence in the plant science community. We thank the Division of Energy Biosciences of the U.S. Department of Energy and administrators at Michigan State University for their funding of the Symposium. More generally, close to half of the financial support for the PRL comes from the Division of Energy Biosciences of the D.O.E., with the remainder coming from Michigan State University, from diverse grants to the faculty, and from fellowships to many of our junioi colleagues. We are very grateful to those who have supported our efforts.

Plant Research '90 summarizes the overall achievements of the PRL during the past year. I prefer that these introductory paragraphs highlight only a few accomplishments, both for their own sake and to illuminate our modus operandi.

Frans de Bruijn arrived from the Max-Planck-Institut für Züchtungsforschung in Köln, Germany, with a vigorous program of studying the $\mathrm{N}_{2}$-fixing association of an unusual tropical legume (Sesbania rostrata, which forms above-ground nodules) and its unusual symbiont (Azorhizobium caulinodans, which can grow on $\mathrm{N}_{2}$ in the free-living state). Focusing on both the plant and the microsymbiont, but especially on their interactions, de Bruijn's group has recently identified regulatory elements controlling nodule-specific expression of leghemoglobin genes, and hitherto unknown loci involved in the control of the nitrogen fixation genes of the bacterium. His group has also made the first observation of the induction of an early nodulin gene by a plant hormone, cytokinin. The reports from the laboratories of Green, Kende and Zeevaart also illustrate that our research into the physiology and metabolism of plant hormones, one of the original foci of research at the PRL, is made ever more fruitful by the extensive cooperation and collaboration between those whos $e$ initial background was more plant-related and those whose initial background was more molecular.

To use the power of genetic analysis to dissect a process, it often helps to find conditions in which the process is not needed. Because the activity of photosystem I had appeared absolutely essential for the growth of every photosynthetic organism whose genome was easily manipulated, genetic analysis of that photosystem had made almost no progress. Thanks to two groups at the PRL, that situation has now changed. Anderson and Mclntosh have shown that 
a transformable unicellular cyanobacterium, Synechocystis sp., may be grown heterotrophically if it be given nhy five minutes of blue light per day; site-directed mutation of a PSI core polypeptide thereupon became possible. My own group has collaborated with groups from Germany and Spain in showing that a strain of the filamentous cyanobacterium, Anabaena, that can grow fully heterotrophically can also be site-specifically mutagenized. In practical terms, these simple results open photosystem I to detailed analysis.

Host-selective fungal toxins have in two recent epidemics proven agriculturally devastating. Although several such toxins were known, the biosynthetic apparatus responsible for their synthesis was elusive. That situation has now changed dramatically through the success of Jon Walton's group in cloning a locus that is responsible for the biosynthesis of the HC-toxin produced by Cochliobolus (Helminthosporium) carbonum. It is pleasing to see one laboratory accomplish both the very difficult biochemistry and the difficult molecular biology necessary to reach this springboard for further studies of diseases that are of major agronomic importance. The work also exemplifies our theme of coupling curiosity-driven research to long-term issues of great practical importance.

The long-range funding that the PRL has had carries with it the obligation to pursue problems that are so stubbornly difficult that they are very unlikely to be resolved by short-range funding. For example, it has been thirty years since Andrew Benson discovered that one of the four major classes of chloroplast lipids was a previously unknown sulfur-containing lipid, present in such abundance as to constitute one of the dominant forms of sulfur in the biosphere. However, in the interim, the pathway of biosynthesis of this lipid, in common with many other features of the anabolic biochemistry and regulation of lipid metabolism in higher plants, has remained unknown. For the sulfolipid, that situation has taken a sharp turn toward elucidation as Christoph Benning, a graduate student working with Chris Somerville, (i) obtained and complemented mutations in the pathway of sulfolipid biosynthesis, (ii) observed that those mutations led to the accumulation of putative intermediates in the pathway, and (iii) developed a cell-free system for synthesis of the sulfolipid from added phosphoadenosine phosphosulfate. In the same laboratory, a stearoyl-ACP desaturase was purified from avocado mesocarp (the first purification of this enzyme), and antibodies to the purified protein were used to clone the corresponding gene from three higher plants. One of the clones produced so much of the protein that study of the mechanism of catalysis by the enzyme is now possible, and is being pursued. These projects illustrate another theme at the PRL: to approach major problems not unidimensionally, but rather, at a variety of levels, often spanning biochemical and molecular genetic approaches.

As this Report goes to press, we have been very pleased to learn that Chris Somerville has received the signal honor of election to fellowship in the Royal Society of London. 


\section{RESEARCH REPORTS}

The following progress reports are not intended as publications and should not be cited without specific permission by the responsible investigator. 


\section{MOLECULAR BASIS OF SYMBIOTIC PLANT-MICROBE INTERACTIONS}

Frans J. de Bruijn. Silvia Rossbach, Johannes Stigter, Maria Schneider, Krzysztof Szczyglowski*, Anurag Goel*, Sylvie Bianchi`, Britta Grunenberg* and Peter Welters*

The induction of nitrogen-fixing root and stem nodules on leguminous plants by soil bacteria belonging to the Rhizobiaceae is a highly evolved process, requiring fine-tuned interaction between the two symbiotic partners. Multiple (regulatory) signals go back and forth between the bacterium and the plant during the formation of an effective nodule (see Long, 1989; Nap and Bisseling, 1990). We are studying the molecular basis of the nodule-specific induction of both rhizobial and plant genes. Our goals include the characterization of the physiological signals and regulatory circuitry controlling free-living versus symbiotic expression of Azorhizobium caulinodans nitrogen-fixation genes, as well as the cis-acting elements and trans-acting factors responsible for nodule-specific expression of plant genes encoding early (Enod2) and late (leghemoglobin, Lb) nodulins. We are also investigating the role of nodule-specific opine-like compounds in rhizobial competition and the potential of using the corresponding synthetic and catabolic genes to create "biased rhizospheres" (see below).

The principal investigator of this task joined the P.R.L. in June of 1990, while remaining coordinator of the research activities of a group of collaborators at the Max Planck Institute in Cologne, F.R.G. for the duration of 1990. Therefore, the work presented was carried out at both locations. The MPI collaborators for the June-December 1990 period are denoted, above, with an asterisk * and were supported by funds from the Max Planck Society.

\section{Regulation of Azorhizobium nitrogen-fixation (nif/fix) genes}

Biological nitrogen fixation is an extremely energy intensive process. In vivo ATP requirements of up to 42 per molecule of $\mathrm{N}_{2}$ reduced have been measured (see O'Brian and Maier, 1989). It is therefore not surprising that free-living nitrogen-fixing organisms only derepress their nif/fix genes when nitrogen-starved ( $N$-regulation; de Bruijn et al., 1990a). Oxygen $\left(\mathrm{O}_{2}\right)$ supports the production of ATP necessary for nitrogenase activity in symbiotically nitrogen-fixing bacteria, but $\mathrm{O}_{2}$ is also capable of severely inhibiting nitrogenase (see Hill, 1988). Therefore, the intracellular $\mathrm{O}_{2}$ concentration represents a second very important environmental signal for rhizobia, which have evolved several mechanisms to regulate their nif/fix genes accordingly $\left(\mathrm{O}_{2}\right.$-regulation; see Hill, 1988; de Bruijn et al., 1990a).

We have been studying $\mathrm{N}$ - and $\mathrm{O}_{2}$ regulation of the nif/fix genes of Azorhizobium caulinodans ORS571 (see de Bruijn et al., 1990a). Strain ORS571 was selected for this analysis, since it has the unique capacity to fix $N_{2}$ in stem and root 
nodules induced on its host, the tropical legume Sesbania rostrata, as well as to grow at the expense of $N_{2}$ in the free-living state (see de Bruijn, 1989). This characteristic makes a proper comparison of nif/fix gene expression in the free-living versus symbiotic state possible. We previously showed that $\mathrm{N}$ - and $\mathrm{O}_{2}$ control of Azorhizobium nif/fix gene expression is mediated primarily via the promoter of the nif-specific positive regulatory gene nifA (Ratet et al., 1989). In the nifA $5^{\prime}$ upstream region we identified specific DNA motifs which have been postulated to be responsible for regulating its expression (Ratet et al., 1989): a nitrogen regulation (ntr) box (-12 to -24; GG-N10-GC; see de Bruijn et al., 1990a), found in genes that require the alternative sigma factor NtrA (RpoN) for their expression under $\mathrm{N}$-starvation conditions, and the fumarate nitrate respiration (fnr) box (TTGAT-N4-ATCAA; see Spiro and Guest, 1990), found in the promoter region of genes anaerobically induced via the transcriptional activator Fnr.

In order to examine the importance of these sequences, site-specific point mutations were constructed, and the resulting promoter regions were fused to the B-galactosidase (lacZ) reporter gene and integrated in the genome of wild-type and (regulatory) mutant strains of Azorhizobium by homologous recombination. Mutations in both the $n t r-$ and $f n r$ motifs were found to drastically affect the expression of the nifA promoter (Figure 1).

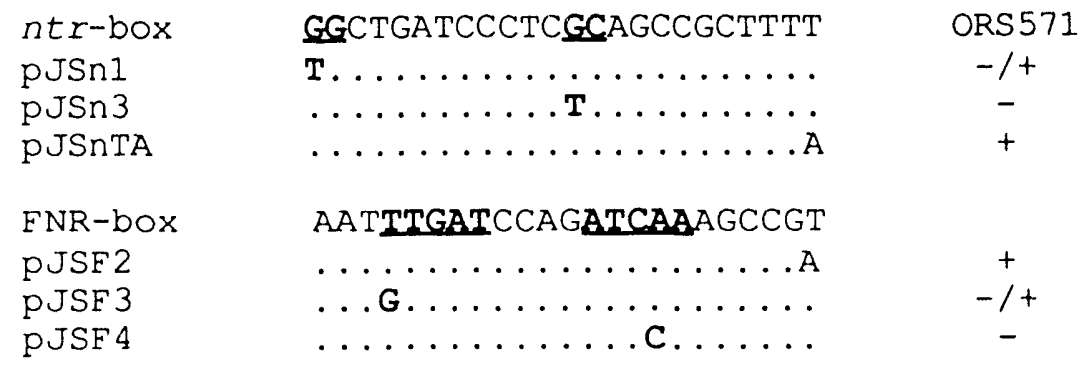

Figure 1. Mutagenesis of the $n t r$ and fnr motifs in the Azorhizobium nifA promoter. The conserved base pairs are underlined and the mutations are indicated below. The effect of the mutations on nifA-lac expressions in Azorhizobium is indicated on the right.

In addition, both the Azorhizobium ntrA and fnr genes were cloned and transposon Tn5 insertion mutations in these loci were created by genereplacement (see de Bruijn, 1987). NtrA::Tn5 mutants were found to be strictly deficient in free-living and symbiotic nitrogen-fixation, supporting the proposed role of NtrA in nif(A) gene regulation. Surprisingly, the fnr::Tn5 mutant was found to be nitrogen-fixation proficient in microaerobic cultures and only slightly impaired in symbiotic nitrogen-fixation. Moreover, a nifA-lacZ fusion was observed to be expressed normally in the fnr::Tn5 mutant background ( $K$. Pawlowski, G. Felix and F.J. de Bruijn, manuscript submitted). Thus, it appears 
that another gene whose product recognized the fnr box may be responsible for $\mathrm{O}_{2}$-control of nif(A) gene expression in Azorhizobium, making the regulatory circuitries for $\mathrm{N}$ - and $\mathrm{O}_{2}$ control in this organism quite complex (see de Bruijn et al., 1990a).

\section{Regulation of S. rostrata early and late nodulin genes}

The group of plant genes which are specifically induced during the symbiotic interaction between rhizobia and their host plant (nodulin genes, Van Kammen, 1984) is commonly divided into "early" and "late" genes. Early nodulins are involved in the infection process and structural aspects of nodule ontogeny. Late nodulins are induced in the mature nodule, around the onset of nitrogen-fixation, and participate in various aspects of nodule functioning, such as oxygen transport, nitrogen assimilation and carbon metabolism (see Nap and Bisseling, 1990). Nodulin genes are interesting plant genes to study, since they are symbiotically induced, developmentally regulated and expressed in a tissue- (cell-) specific manner (see Nap and Bisseling, 1990; de Bruijn et al., 1990b). The regulation of expression of the early and late nodulin genes differs substantially, but the nature of the (rhizoblal) regulatory signals and their targets in the nodulin gene promoter regions is only beginning to be understood (see de Bruijn et al., $1990 \mathrm{~b})$. We have chosen the early nodulin gene Enod2 and the gene family encoding the oxygen carrying leghemoglobin (Lb) proteins for our analysis.

\section{The Enod2 gene is induced by cytokinin}

The $S$. rostrata Enod2 gene is expressed around 8 days after infection and encodes a proline-rich protein, consisting predominantly of two repeating oligopeptides [XEKPP(P); $X Y(X) P P(P)]$ and a putative signal peptide (C. Dehio and F.J. de Bruijn, manuscript submitted). The Enod2 gene has been shown to be specifically expressed in the nodule parenchyma cells (Van de Wiel et al., 1990 ) and the Enod2 product has been proposed to be a cell-wall protein, which may play a role in the creation of an oxygen diffusion barrier in the nodule (see Nap and Bisseling, 1990). This would represent a second mechanism to deal with the oxygen problem in the nodule. We have started to examine the regulation of the $S$. rostrata Enod2 gene by using northern hybridizations and by analysing the expression of chimeric Enod2-B-glucuronidase (uidA, gus) genes in transgenic Lotus corniculatus plants (see de Bruijn et al., 1990b). During the process of generating transgenic Lotus and Sesbania tissues via Agrobacterium rhizogenes, we observed that the endogenous Enod2 genes were expressed in hairy roots in the absence of infecting rhizobia. The $S$. rostrata Enod2 gene was also found to be expressed in tumors induced by wild-type, but not by cytokinin biosynthesis deficient mutants of $\boldsymbol{A}$. tumefaciens. These observations suggested a role for cytokinin in Enod2 induction. In fact, we have been able to show that exogenous application of physiologically significant concentrations of cytokinins (e.g. 10nM zeatin) induces Enod2 gene expression in S. rostrata roots within $2 \mathrm{~h}$ (Figure 2). 
This response is cytokinin-specific and not found in auxin-, gibberellin- or ethylene-treated plantlets, nor in plantlets stressed by heat, flooding, desiccation, wounding or heavy metals (C. Dehio and F.J. de Bruijn, manuscript submitted).

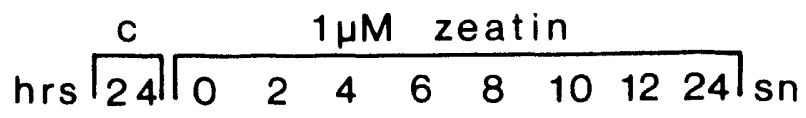

Figure 2. Cytokinin induction of the Sesbania Enod2 gene. Nothern blot of root RNA of Sesbania seedlings at different timepoints (in hours) after applying $1 \mu \mathrm{M}$ zeatin, hybridized with the cloned Enod2 gene. "Sn" indicates Sesbania stem nodule RNA.

This gene is therefore not only a good candidate to study nodule-specific plant gene expression, but also serves as a unique model to study plant gene induction by cytokinin. We are presently investigating whether rizobial cytokinin production plays a direct role in Enod2 gene induction and whether the cis-acting elements in the Enod2 promoter region responsible for nodule (parenchyma)-specific expression correspond to those involved in cytokinin induction.

Cis-acting elements involved in nodule-specific expression of leghemoglobin genes

The leghemoglobin $(\mathrm{lb})$ genes are maximally induced about 4 days after the Enod2 genes and encode oxygen carrying proteins, which facilitate oxygen diffusion to the actively respiring, nitrogen-fixing bacteroids within the infected zone of the nodule. Lbs operate at an intracellular $\mathrm{O}_{2}$ concentration of $10 \mathrm{nM}$, which is below the concentration known to irreversibly inactivate the nitrogenase enzyine complex (Appleby, 1984). This constitutes a third mechanism to deal with the $\mathrm{O}_{2}$ problem in nitrogen-fixing nodules.

A functional analysis of the $S$. rostrata leghemoglobin $g / b 3$ gene promoter region in transgenic Lotus and tobacco plants has revealed the presence of several positive regulatory regions and an ATG-proximal, tissue-specific element involved 
in nodule-specific gene expression (de Bruijn et al., 1990b; Szabados et al., 1990; "+" and "nod" respectively, Figure 3). Cytological Gus staining experiments have revealed that the g/b3 promoter is only expressed in the infected cells of the nodule (Szabados et al., 1990). Moreover, the g/b3 promoter has been found to be expressed in tobacco, primarily in the roots, which is interesting in light of the fact that the hemoglobin gene of the non-nodulated plant Trema tomentosa also shows a root-specific expression pattern in tobacco (Bogusz et al., 1988; Szabados et al., 1990).

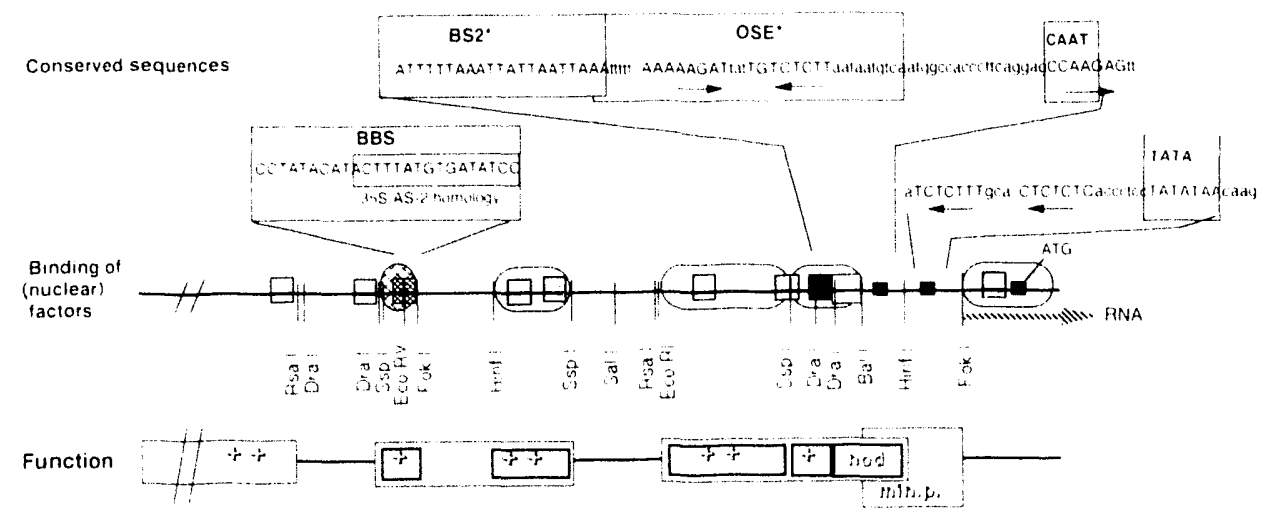

Legend
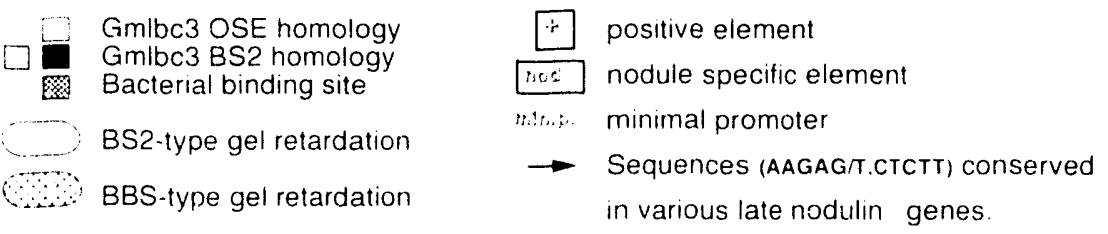

Figure 3. Cis-acting elements in the Sesbania g/b3 5 ' upstream region and interactions with trans-acting factors. For details see text.

The DNA sequences responsible for nodule-specific expression have been delimited to a 78-bp DNA fragment (position -147 to 69 ; Figure 3; $\mathrm{K}$. Szczyglowski, L. Szabados, P. Ratet and F.J. de Bruijn, manuscript in preparation). The corresponding regions of the soybean and $S$. rostrata $\mathrm{lb}$ gene promoters share a high degree of homology (Metz et al., 1988). This homology includes the Ibc3 OSE (Organ Specific Element) region, with the typical AAAGAT and CTCTT motifs found in several late nodulin gene promoters (see de Bruijn et al., 1990b; Forde et al., 1990; Figure 3). The AAAGAT-like motif has also been found in the $5^{\prime}$ upstream regions of the hemoglobin genes from Parasponia (nodulated non-legume) and Trema (non-nodulated relative of Parasponia; Bogusz et al., 1990). The latter observation is interesting, since the Trema hemoglobin gene promoter directs nodule-enhanced expression of a reporter 
gene in transgenic Lotus plants (Bogusz et al., 1990). The interaction of the OSE* region of the g/b3 gene with further upstream elements is under investigation and site-specific mutagenesis experiments are in progress to elucidate the role of specific base-pairs of the conserved motifs in nodule-specific gene expression (This work was supported, in part, by USDA grant 90-37262-5781.)

\section{Trans-acting factors interact with lb gene 5' upstream regions}

We previously reported the specific binding of a nodule factor to an $A / T$-rich binding site, repeated several fold in the $S$. rostrata g/b3 and soybean $/ b c 35^{\prime}$ upstream regions (Jensen et al., 1988; Metz et al., 1988; de Bruijn et al., 1989; BS2* and shaded squares, Figure 3).

We have found that an oligonucleotide corresponding to this $A / T$ binding site (BS2*) is capable of enhancing gene expression in transgenic plants, when fused in cis to a minimal plant promoter, derivey from the nopaline synthase gene ( $P$. Welters, K. Szczyglowski, L. Szabados and F.J. de Bruijn, unpublished resul'(s), suggesting a role for these sequences in vivo. However, this enhanced expression is not nodule-specific, confirming the requirement for other cis-acting elements (see above). Gel-retardation assays have also revealed a distinct binding site that is not $A / T$-rich, approximately 620 bp upstream of the transcriptional start-site (BBS, Figure 3), which interacts specifically with a heat stable, proteinaceaous factor from nodule extracts. Surprisingly, this factor was found to be produced by the infecting Azorhizobium (P. Welters and F.J. de Bruijn, unpublished results). One of the proteins co-purifying with the binding activity $(11 \mathrm{kD})$ was partially sequenced and found to share a high degree of homology with histones and bacterial HU proteins (P. Welters, K. Palme and F.J. de Bruijn, unpublished results). Bacterial $\mathrm{HU}$ proteins are involved in gene transcription, possibly by bending DNA (see Schmidt, 1990). An oligonucleotide carrying this binding site (BBS) has been found to enhance the general expression of a minimal plant promoter in transgenic plants when fused to it in cis, just as observed for the $A / T$-rich binding sites ( $P$. Welters, $L$. Szabados and F.J. de Bruijn, unpublished results), but the role of this peculiar protein-DNA interaction in $l b$ gene expression remains to be elucidated.

Thus, multiple cis-acting eiements and trans-acting factors play a role in $\mathrm{lb}$ gene regulation (see de Bruijn et al., 1990b) and the architecture of the $/ \mathrm{b} 5^{\prime}$ upstream regions is correspondingly complex (Figure 3). Experiments to clone the genes encoding the trans-acting factors and further characterize their binding sites are in progress to elucidate the signal transduction pathway involved in nodule-specific plant gene expression. 


\section{Rhizobial opine biosynthetic and catabolic genes and the creation of "biased rhizospheres"}

We have previously described the isolation of a nodule-specific, opine-like compound from alfalfa nodules induced by Rhizobium meliloti strain L5-30 (Murphy et al., 1987). This compound was designated "opine-like" by analogy with Agrobacterium, since only the strain that induced its synthesis could use it as growth substrate. Unlike in the case of Agrobacterium, the rhizobial opine (rhizopine) was found to be synthesized by the bacterium and both the rhizopine synthetic (mos) and catabolic (moc) genes were shown to be closely linked on the symbiotic plasmid (Murphy et al., 1987). In fact, the mos genes were found to be symbiotically induced (in the bacteroids) via the nif-regulatory protein NifA (see above), revealing a close linkage between the two processes (Murphy et al., 1988). The moc and mos genes have been cloned (Murphy et al., 1987; 1988) and the DNA sequence of the mos locus has been determined (S. Trenz, F. J. de Bruijn, J. Schell and P. Murphy, unpublished results). We are interested to use the moc/mos system to create "biased rhizospheres" and to study the persistence/competition of genetically engineered organisms in the soil. To achieve the former, we will engineer a (crop) plant of interest to produce rhizopine specifically in the roots, introduce a moc cassetie into beneficial soil bacteria and monitor their competitive ability in the rhizosphere of the rhizopine producing plant. To prepare the moc cassette, we have mapped the moc locus and constructed moc-nptll fusions to monitor promoter strength and regulation. To investigate the specificity of the system, we are screening a large number of different $R$. meliloti strains and other soil bacteria for the presence of moc/mos sequences and ability to degrade the rhizopine. For the latter purpose, we are collaborating with Dr. R. Hollingsworth (MSU Department of Biochemistry), who is synthesizing the rhizopine (3-O-methyl-scyllo-inosamine; 3-O-MSI). This work is supported by the MSU Center for Microbial Ecology.

\section{References}

Bogusz, D., C.A. Appleby, J. Landsmann, E.S. Dennis, M.J. Trinick and W.J.

Peacock. Nature 331:178 (1988).

Bogusz, D., D. J. Lewellyn, S. Craig, E. Dennis, C. Appleby and W.J. Peacock. The Plant Cell 2:633 (1990).

de Bruijn, F.J. Meth. Enzymol. 154:175 (1987).

de Bruijn, F.J. In: Plant-Microbe Interactions: Molecular and Genetic

Perspectives (T. Kosuge and E. Nester, Eds.), MacMillan, New York, pp. 457-493 (1989).

de Bruijn, F.J., G. Felix, B. Grunenberg, H-J. Hoffmann, B. Metz, P. Ratet, A.

Simons-Schreier, L. Szabados, P. Welters and J. Schell. Plant Mol. Biol. 13:319 (1989).

de Bruijn, F.J., U. Hilgert, J. Stigter, M. Schneider, H. Meyer Z.A., U. Klosse and

K. Pawlowski. In: Nitrogen Fixation: Achievements and Objectives (P. Greshoff, 
E. Roth, G. Stacey and W.E. Newton, eds.), Chapman and Hall, New York, pp. 33-44 (1990a).

de Bruijn, F.J., L. Szabados ard J. Schell. Dev. Genet. 11:182 (1990b).

Forde, B.G., J. Freeman, J.E. Oliver and M. Pineda. The Plant Cell 2:925 (1990).

Hill, S. FEMS Microbiol. RGv. 54:111 (1988).

Jensen, E.O., K. Marcker, J. Schell and F.J. de Bruijn. EMBO J. 7:1265 (1988). Long, S.R. Cell 56:203 (1989).

Metz, B. A., P. Welters, HJ. Hoffmann, E.O. Jensen, J. Schell and F.J. de Bruijn. Mol. Gen. Genet. 214:181 (1988).

Murphy, P.J., N. Heycke, Z. Banfalfi, M.E. Tate, F.J. de Bruijn, A. Kondorosi, J. Tempe and J. Schell. Proc. Natt. Acad. Sci USA 84:493 (1987).

Murphy, P.J., N. Heycke, S.P. Trenz, P. Ratet. F.j. úe Bruijn and J. Schell. Proc. Natl. Acad. Sci. USA Ej:9133 (1988).

Nap, J-P. and T. Bisseling. Science 250:948 (1990).

O'Brian, M.R. and R.J. Maier. Biochim. Biophys. Acta 974: 229 (1989).

Ratet, P., K. Pawlowski, J. Schell and F.J. de Bruijn. Mol. Microbiol. 3:825 (1989).

Schmidt, M.B. Cell 63:451 (1990).

Spiro, S. and J.R. Guest. FEMS Microbiol. Rev. 75:399 (1990).

Szabados, L., P. Ratet, B. Grunenberg and F.J. de Eruijn. The Plant Cell 2:973 (1990).

Van de Wiel, C., B. Scheres, H. Franssen, M-J. van Lierop, A. van Lammeren, A. van Kammen ard T. Bisseling. EMBO J. 9:1 (1990).

Van Kammen, A. Plant Mol. Biol. Rep. 2:43 (1984). 


\title{
ENZYMATIC MECHANISMS AND REGULATION OF PLANT CELL WALL BIOSYNTHESIS
}

\author{
Deborah P. Delmer. Andrawis Andrawis, Yehudit Amor, Liat Gonen, Mazal \\ Solomon, Esther Shedletzky, Miri Shmuel and Tali Trainin (The Hebrew \\ University)
}

This has been an exciting year for those of us who have dedicated years of efforts toward understanding the mechanism of cellulose biosynthesis. One major breakthrough in this field has come with two reports of cloning of genes involved in the process in the cellulose-secreting bacterium Acetobacter xylinum (Wong et al., 1990; Saxena et al., 1990). This organism has proven exceedingly useful for the study of cellulose synthesis since, unlike the situation in higher plants, high activity for the cellulose synthase can be detected in vitro, due to the discovery of a unique activator of the enzyme, cyclic-di-guanylic acid (c-di-GMP) (Ross et al., 1987). This year, our group has made the exciting discovery of several cotton fiber membrane polypeptides which bind c-di-GMP and bear striking analogy to the bacterial cellulose synthase, thus opening the way at last for understanding the nature of the elusive cellulose synthase of plants.

In the course of our continuing studies on both cellulose and callose synthesis in cotton fibers, we have also discovered that $\mathrm{Ca}^{2+}$ plays a complex role in determining how certain polypeptides interact with the plasma membrane, and that such regulation may be important for control of glucan synthase activities. We also report here our initial results on attempts to select mutants of Arabidopsis impaired in glucan synthesis.

We have also continued our studies on a unique cell line of tomato which is adapted in culture for growth on the cellulose-synthesis inhibitor, 2,6dichlorobenzonitrile (DCB). Last year we showed that when these cells grow on DCB, the cell walls virtually lack a cellulose-xyloglucan network (Shedletzky et al., 1990). Since this is a major load-bearing network in dicot walls, we are now concentrating our efforts on determining the structure of the residual wall of these cells in order to understand what compensatory changes have been made to adjust for loss of this network.

Since the leader of this task (D.P.D.) is a Professor at The Hebrew University in Jerusalem and an Adjunct Professor at the PRL, research is annducted in both locations.

\section{Evidence for a c-di-GMP-dependent cellulose synthase in plants}

Although c-di-GMP is a very specific activitor of the bacterial cellulose synthase, no evidence has been available concerning its possible role in plants. The uniqueness of its structure and function makes it an attractive probe to search for 
any polypeptides in plants which may bear similarity to the bacterial cellulose synthase. Therefore, in collaboration with R. Mayer and M. Benziman of The Hebrew University, we have synthesized ${ }^{32} \mathrm{P}$-c-di-GMP and used it for direct photo-labeling of cotton fiber membrane polypeptides. The data of Fig. 1 show that two c-di-GMP-binding polypeptides, of 83 and $48 \mathrm{kD}$ can be identified with this probe.

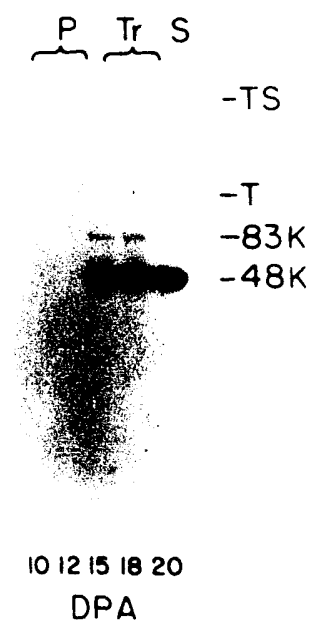

Figure 1. Developmental pattern of photo-labeling of cotton fiber membrane polypeptides with ${ }^{32}$ P-C-di-GMP. P, Tr, and $S=$ stages of primary, transition, and secondary cell wall synthesis, respectively. DPA = days post-anthesis of fibers used to prepare extracts for photo-labeling. $T S=$ stop of stacking gel; $T=$ top of separating gel.

The ability to detect these c-di-GMP polypeptides is strongly correlated with the increase in the rate of cellulose synthesis in vivo. Neither polypeptide can be detected by photo-labeling of membrane proteins derived from fibers engaged in low rates of cellulose synthesis during primary wall deposition; both are first detected at the transition to secondary wall deposition, and the ability to label the 48-kD species increases markedly in fibers actively engaged in secondary wall cellulose synthesis. In these fibers, the raie of synthesis in vivo is $>100$-fold higher than that found in young fibers (Meinert and Delmer, 1977).

These polypeptides show high affinity for c-di-GMP (24 and $13 \mathrm{uM}$ for the 83-and 48-kD polypeptides, respectively). They also show a high specificity of interaction with this probe. Of a variety of related nucleotides tested as competitors, only 5'GMP (which mimics half of the C-di-GMP molecule) and c-di-IMP, also an effective activator of the bacterial enzyme (Ross et al., 1990), effectively competed for labeling. Other nucleotides and cyclic nucleotides which are ineffective in the bacterial system were found to have little or no affinity for the cotton fiber 
polypeptides. Further strengthening the argument that these polypeptides are components of a cellulose synthase is our observation that both show crossreaction during Western blotting with a polyclonal antibody prepared against a subunit of the bacterial synthase. Since both the 83- and 48-kD species crossreact, we tentatively conclude that the $48 \mathrm{kD}$ is a proteolytic fragment of the 83 KD polypeptide, a situation analogous to that found in $A$. xylinum where a 91-kD primary gene product is cleaved to a 67-kD c-di-GMP-binding fragment (Mayer et al., 1991).

In sum, these observations lead us to the conclusion that higher plants very likely contain a c-di-GMP-dependent cellulose synthase which bears remarkable similarity to the bacterial enzyme. These findings offer hope that the genes coding for the synthase soon can soon be identified using sequence information derived from clones of the bacterial genes.

\section{In search of the catalytic subunit of cotton fiber cellulose synthase}

The question now arises as to whether the c-di-GMP-binding polypeptides described above also contain a site for binding of the synthase substrate UDPglc. In previous work (Delmer et al., 1991), we showed that an 84.kD cotton fiber membrane polypeptide was one of several which can be photo-labeled with ${ }^{32} \mathrm{P}$. UDF-glc. This polypeptide migrates nearly, but not exactly, coincident with the 83-kD c-di-GMP-binding species during SDS-PAGE, and, in most, but not all, experiments, labeling with UDP-glc is markedly enhanced by the addition of unlabeled C-di-GMP, suggesting that it may indeed by the catalytic subunit of the synthase. We now have further evidence that the UDP-glc- and c-di-GMPbinding polypeptides are distinct species which do not exactiy co-migrate during gradient centrifugation.

A comparison of our data with cotton fibers with recently published work on $A$. xylinum leads us to consider a model wherein the cellulose synthase is comprised of two types of non-identical subunits, one of which is an 84-kD catalytic subunit binding UDP-glc, and the other of which is a c-di-GMP-binding regulatory subunit of $83 \mathrm{kD}$ (cleaved to $48 \mathrm{kD}$ ). Analyzing the recent work on $A$. xylinum, one can also propose a similar model for the bacterial enzyme. Wong et al. (1990) cloned an operon of four genes proposed to be involved in cellulose synthesis, and concluded that the second geria (bcsB) coded for the catalytic subunit based primarily upon genetic evidence. Further biochemical studies (Mayer et al., 1991) make clear that this gene codes for a polypeptide of $91 \mathrm{kD}$ which is cleaved to a 67-kD c-di-GMP-binding protein, but was less conclusive as to whether a 54-kD UDP-glc-binding polypeptide also found in purified synthase preparations was also derived from this gene. Saxena et al. (1990) cloned a gene based upon the sequence of in 83-kD UDP-glc-binding polypeptide found in their most pure preparations of the bacterial synthase. Interestingly, this gene shows very high homology with the first gene (bscA) of the operon cloned by Wong et al. (1990). 
Thus, it seems most logical to conclude that the A gene codes for the catalytic subunit and the $B$ gene for a regulatory subunit of the synthase. The function of the other two genes of the operon is unknown, and it will be of interest to know if they have counterparts in plants.

\section{The effect of $\mathrm{Ca}^{2+}$ on localization and function cotton fiber plasma membrane proteins.}

In our continuing studies on callose synthesis in cotton fibers, we have observed that activity for callose synthase is very high when extracts are prepared in the presence of $25 \mathrm{mM}$ EDTA and much lower when membranes are isolated in the presence of low levels of $\mathrm{Ca}^{2+}$. Subsequent washing of the latter membranes with EDTA restores callose synthase activity to high levels and also releases a characteristic set of proteins to the supernatant. The most notable of the polypeptides released are a very abundant 34-kD polypeptide and the 48-kD c-diGMP-binding protein described above.

1. The 34-kD polypeptide has the following properties: a) it can be photo-labeled with $\alpha-{ }^{32}$ P-GTP; b) it can also be labeled with an analog of UDP-glc, UDP- $-\mathrm{H}$ pyridoxal; $c$ ) it can be precipitated in almost pure form by addition of $\mathrm{Ca}^{2+}$ (due to this latter characteristic, it is also found in abundance in pellets during product entrapment of callose synthase: see last year's Annual Report); and d) in many, but not all, experiments re-addition of the 34-kD species to preparations of gradient-purified callose synthase results in marked inhibition of enzyme activity. In terms of its size and $\mathrm{Ca}^{2+}$-dependent interaction with the plasma membrane, this polypeptide resembles mammalian proteins called annexins which are thought to play a variety of regulatory roles including facilitating interaction of the cytoskeleton with the plasma membrane, secretion, and inhibition of phospholipase $A_{2}$ activity (Crompton et al., 1988). An annexin-like protein has recently been characterized in tomato (Smallwood et al., 1990).

2. To our surprise, the C-di-GMP binding protein is released to the supernatant when membranes are prepared in EDTA. This release, and the protein's proposed origin by proteolytic cleavage from an 83-kD precursor, may offer some clues as to why cellulose synthase activity has not been detected previously.

These findings suggest that changes in intracellular $\mathrm{Ca}^{2+}$ levels may regulate glucan synthesis in very complex ways in addition to the well-known $\mathrm{Ca}^{2+}$ activation of callose synthase (Delmer, 1987; Kauss, 1987). This year we have also observed that the size of a soluble polypeptide which interacts with DCB is markedly different when extracts are prepare $d$ in the presence of EDTA (42 kD) or divalent cations (18 kD). A further intriguing observation is that a soluble $\mathrm{Ca}^{2+}$. dependent protein kinase activity is only detected in extracts prepared in the presence of $\mathrm{Ca}^{2+}$ and is totally absent in extracts prepared in EDTA. In the next year we hope to understand more clearly the structure and function of the 34-kD 
polypeptide as well as the physiological significance of the effects of divalent cations on the interaction of the various polypeptides with the plasma membrane. Toward these ends, we have already prepared a polyclonal antibody against the 34-kD polypeptide and are engaged in attempts to sequence peptides derived from it.

\section{A search for mutants of Arabidopsis impaired in glucan synthesis.}

Callose is not a normal wall constituent except in specialized cells such as pollen tubes or fibers derived from epidermal hairs of seeds. It is transiently deposited at the cell plate, and synthesized rapidly and in large amounts at sites of wounding or infection (Delmer, 1987; Kauss, 1987). Thus, the interesting question arises as to whether a plant impaired in callose synthesis could survive and what its phenotype would be. This year we devised a rapid screening procedure used for attempts to select such mutants in Arabidopsis. Pieces of leaves of young $\mathrm{M}_{2}$ Arabidopsis seedlings are incubated with substrate and effectors of callose synthase, permeabilized with DMSO, and callose produced by trichomes is detected by fluorescence microscopy following staining with Sirofluor. After screening 400 such seedlings, one plant deficient in its ability to synthesize callose was tentatively identified, its seed collected, and its progeny are now being further analyzed. A search for more such mutants is in progress.

\section{The structure of cell walls of DCB-adapted suspension-cultured tomato cells}

The mechanism of adaptation of tomato cells to growth on the cellulose-synthesis inhibitor DCB is somewhat surprising. Instead of selecting cells with a pathway of cellulose synthesis resistant to DCB, we found that our adapted cells tolerated DCB because they had "learned" to grow with cell walls containing almost no cellulose (Shedletzky et al., 1990). This year we learned that these cells still possess the capacity for cellulose synthesis and proceed to do so at normal, or even higher than normal, rates immediately after removal of DCB. We also now know that this type of adaptation is not unique to tomato, since we now have an adapted cell line of tobacco which has a similar altered wall composition when growing on DCB.

Our early studies on the nature of the residual wall of tomato cells growing on $D C B$ revealed that these cells also had reduced levels of hyp-rich protein, elevated levels of uronic acid-rich polymers, and very reduced levels of xyloglucan which, lacking cellulose with which to interact, was excreted to the medium in large amounts. Since EDTA treatment of walls did not release a substantial amount of polymers, but subsequent treatment with very mild alkali did, we concluded that ester linkages might be important in linking some of the major residual uronic-acid-rich polymers (Shedletzky et al., 1990). This year, we have somewhat modified this conclusion with the finding that, if CDTA is substituted for EDTA as chelator, the walls of intact cells break and substantial 
amounts of uronic-acid-rich polymers are released. From this finding, we now conclude that the interaction of these polymers with $\mathrm{Ca}^{2+}$ provides one of the major load-bearing network in these walls.

Nevertheless, a substantial part of the wall still remains insoluble after CDTA treatment, and we have devoted further effort toward determining the linkages between polymers in this fraction as well. In searching for ester linkages, we treated walls with $\mathrm{NaBH}_{4}$ in imidazole buffer at $\mathrm{pH} 7.0$ and $4 \mathrm{C}$ to selectively reduce uronic acids in ester linkage (Maness et al., 1990). To our surprise, very little carbohydrate or uronic acid was released, but about $25 \%$ of the wall protein was released. Following dialysis and lyophilization, one specific SDS-soluble protein of $23 \mathrm{kD}$ was found which was not rich in hyp, pro, or gly. A substantial amount of protein remained insoluble in SDS, and when analyzed, was found to have an amino acid composition resembling classic extensins with ser-hyp $p_{4}$ repeats. In walls from non-adapted cells, the 23-kD protein was released in higher amounts, while the insoluble extensin was released in lesser amounts compared to walls of adapted cells growing on DCB. From these results, we tentatively conclude that tomato cells may contain a cross-linked extensin network which is linked to other polymers in the wall via ester linkages; the level of this network may be relatively higher in the walls of adapted cells.

\section{References}

Crompton, M.R., S.E. Moss, and M.J. Crumpton. Cell 55:1 (1988).

Delmer, D.P. Ann. Rev. Plant Physiol. 38:259 (1987).

Delmer, D.P., M. Solomon, and S.M. Read. Plant Physiol., in press (1991).

Kauss, H. Ann. Rev. Plant Physiol. 38:47 (1987).

Mayer, R., P. Ross, H. Weinhouse, D. Amikam, G. Volman, P. Ohana, R. Calhoon, H.C. Wong, and M. Benziman. Proc. Natl. Acad. Sci. USA, in press.

Maness, N.O., J.D. Ryan, and A.J. Mort. Analyt. Biochem. 185:346 (1990).

Meinert, M. and D.P. Delmer. Plant Physiol. 59:1088 (1977).

Ross, P., H. Weinhouse, Y. Aloni, D. Michaeli, P. Weinberger-Ohana, R. Mayer, S. Braun, E. de Vroom, G.A. van der Marel, J.H. van Boom, and M. Benziman. Nature 325:279 (1987).

Ross, P., R. Mayer, H. Weinhouse, D. Amikam, Y. Huggirat, M. Benziman, E. de Vroom, A. Fidder, P. de Pans, S. Sliedregt, G.A. van der Marel, and J. H. van Boom. J. Biol. Chem. 265:18933 (1990).

Saxena, I.M., F.C. Lin, and R.M. Brown, Jr. Plant Mol. Biol. 15:673 (1990).

Shedletzky, E., M. Shmuel, D.P. Delmer, and D.T.A. Lamport. Plant Physiol. 94:980 (1990).

Smallwood, M., J.N. Keen, and D.J. Bowles. Biochem. J. 270:157 (1990).

Wong, H.C., A.L. Fear, R.D. Calhoon, G.H. Eichinger, R. Mayer, D. Amikam, M. Benziman, D.H. Gelfand, J.H. Meade, A.W. Emerick, R. Bruner, A. Ben-Bassat, and R. Tal. Proc. Natl. Acad. Sci. USA 87:8130 (1990). 


\title{
MOLECULAR MECHANISMS THAT REGULATE THE EXPRESSION OF GENES IN PLANTS
}

\author{
Pamela J. Green. Thomas C. Newman, Pedro Gil, Christie Howard, Yang Liu, \\ Masaru Takagi, Crispin Taylor, and Yang Yen
}

The steady state level of an mRNA depends both on its rate of synthesis (transcription) and its rate of degradation. Rapidly accumulating data indicates that degradation rates of mRNAs in eukaryotes vary over a wide range and can be regulated by a variety of stimuli. However, in contrast to transcription, the mechanisms that control mRNA stability are largely unknown. A major objective of our work is to identify and characterize the molecular components that control the rates of mRNA degradation in plants (e.g., RNases and the mRNA sequences that they recognize) and determine how they interact. Another goal is to understand how certain components respond to plant growth regulators and to environmental stimuli so as to alter selectively the rates of mRNA degradation. Our work will make maximal use of decay measurements in transgenic plants and the isolation and analysis of mutants because such experiments are not feasible in animal model systems where much of the pioneering work on mRNA stability has been done. As such this work has the potential to identify new components that are unique to plants or of broad significance to higher eukaryotes. Beyond its contribution to basic knowledge, this research should suggest solutions to practical problems resulting from the instability of foreign mRNAs in genetically engineered crop plants.

Development of methods to measure rates of mRNA degradation in suspension cultures. To date no system has been reported for the measurement of the degradation rates of specific transcripts in stably transformed plant cells. The development of such systems will allow the stabilities of a variety of engineered transcripts to be directly compared. We have sought to establish methods to measure mRNA degradation rates in a cultured line of Nicotiana tabacum cells (NT-cells) (Nagata et al., 1981) based on methods used by others for cultured mammalian cells (e.g. Lindsten et el., 1989; Schuler and Cole, 1988). NT-cells provide an attractive model system for such an endeavor because they are homogeneous, grow rapidly and are very easy to manipulate. Moreover, transformation of NT-cells via Agrobacterium tumafaciens is particularly expedient (An, 1985). Our initial efforts have focused on the use of inhibitors of transcription to allow degradation of the mRNA to be studied in the absence of synthesis. Very few experimental data are airailable on the effectiveness of potential transcriptional inhibitors in plant cells. For this reason we tested several inhibitors for their ability to reduce the incorporation of ${ }^{3} \mathrm{iH}$-uridine into polyadenlyated mRNA in NT-cells. These experiments showed that Actinomycin D (ActD), which is the most commonly used transcriptional inhibitor for the study of mRNA stability in mammalian cells, effectively inhibits mRNA synthesis in NT-cells. $\alpha$ amanitin, which is known to inhibit transcription in isolated plant and animal 
nuclei, had no effect on mRNA synthesis in intact cells. The lack of effect of $\alpha$ amanitin, and another potential inhibitor, cordycepin, may indicate that these compounds do not enter intact NT-cells efficiently. Using several probes specific for endogenous genes or foreign genes stably introduced into NT-cells, we have identified candidates for inherently unstable and inherently stable mRNAs. The measurement of mRNA decay rates for the unstable mRNAs following ActD treatment is in progress. Our data indicates that the kinetics of mRNA degradation are first order over the course of our experiments. To measure the degradation rates of long-lived mRNAs, a pulse-chase method will be developed for use with NT-cells. (Supported, in part, by the USDA grant \# 9001167.)

Reporter genes for the identification of sequences that control mRNA stability. An effective way to identify sequence elements that control gene expression is to test putative regulatory elements for function within the context of a foreign reporter ("TEST") gene. We have constructed a TEST gene consisting of the strong $35 S$ promoter from the Cauliflower Mosaic Virus, fused to the coding region of the bacterial $\beta$-glucuronidase gene (GUS), and polyadenylation signals from the rbcS-E9 gene (E9). To facilitate the insertion of putative regulatory elements, the promoter, coding region, and 3 ' end of the 35S-GUS-E9 TEST gene are separated with unique restriction sites. The sites flanking the GUS segment are located in the $5^{\prime}$ and $3^{\prime}$ noncoding regions of the transcription unit where sequences that affect mRNA stability are known to reside. In mammalian cells, an $A U$-rich sequence from an unstable lymphokine transcript was shown to destabilize the $\beta$-globin transcript when inserted into the $3^{\prime}$ noncoding region of the mRNA (Shaw and Kamen, 1986). This AU-rich element and several others have been incorporated into the 35S-GUS-E9 TEST gene in either the $3^{\prime}$ or $5^{\prime}$ noncoding region. Each of the modified TEST genes has been introduced into NT-cells using a transformation vector containing a 35S-CAT-3C REFERENCE gene that will serve as an internal standard for all constructs. Because our experiments indicate that mRNA decay is first order, position effects that alter the rate of transcription should not alter the rate of decay. Nevertheless, our reporter gene configuration is very similar to a test/reference gene system used previously to normalize for position effects in transcriptional studies (Fang est al., 1989). Our preliminary data indicates that control CAT and GUS transcripts decay with similar half-lives in NT cells. Half-life measurements for GUS transcripts containing putative instability determinants is in progress.

A second set of reporter genes has been constructed to test whether sequence elements that regulate mRNA stability in NT-cells play the same roles in transgenic plants. In plants, we will use a regulated promoter from a chlorophyll $\mathrm{a} / \mathrm{b}$ binding protein gene (Cab-1) to control transcription rather than the $35 \mathrm{~S}$ promoter used for NT-cells. The Cab-1 promoter is regulated by light and a circadian clock such that it directs transcription transiently each morning in transgenic tobacco (Nagy et al., 1988). Therefore it should be possible to follow the decay of transcripts driven by the Cab promoter by isolating RNA from leaf 
samples during the afternoon and evening after transcription has ceased. Control Cab-GUS-E9 and Cab-CAT-3C genes have been introduced into tobacco, and transgenic plants have been regenerated. Preliminary experiments have shown that the CAT and GUS transcripts disappear in the afternoon and evening as expected. F1 seedings from the transgenic plants are being used to determine more precisely how fast these transcripts decay. While in theory, any promoter that can be induced transiently (e.g. a heat-shock promoter) could be used in this manner, the Cab-1 promoter offers the unique advantage of not requiring any alteration of normal growth condikions while stability is examined. (Supported, in part, by USDA grant \#9001167.)

Identification of Major Ribonucleases (RNases) from Arabidopsis. We have used a substrate-based gel assay characterize the profile of RNases in Arabidopsis, as a first step towards identifying the RNases that play a role in mRNA stability. Using this approach, we have identified up to 16 RNases from Arabidopsis. These RNases vary in size from 9 to $41 \mathrm{kD}$. Most of the RNases exhibit a pH optimum of about 6.5, however an activity at $22.6 \mathrm{kD}$ is enhanced at low pH. A number of RNases in the 30- to 41-kD range are sensitive to EDTA and their activities are enhanced by the presence of a low concentration of zinc during renaturation. At least one RNase appears to co migrate with a major DNase activity. The differential accumulation of several RNases in stems versus leaves indicates that some RNases are controlled in an organ-specific manner in Arabidopsis. It should now be possible to use the gel assay to screen M2 Arabidopsis plants for mutants that lack any of the major RNases that have been identified. (Supported, in part, by a grant from the McKnight foundation.)

Identification of Putative RNase Genes from Arabioopsis. The only RNase genes that have been cloned from higher plants are those expressed in species known to exhibit self-incompatibility, such as the S-genes of Nicotiana alata (McClure et al., 1989). We have investigated whether this particular type of RNase gene is restricted to self-incompatible species, or if similar genes are expressed in other plants. Using a polymerase chain reaction we have identified a set of three putative RNase genes in Arabidopsis, which a self-compatible plant (Green and Taylor, 1991). These Arabidopsis genes, designated RNS1, RNS2, and RNS3, do not cross hybridize to each other and their products are homologous to both the S-gene RNases (McClure et al. 1989) and a set of fungal enzymes (Horiuchi et al. 1988; !ria et al. 1986) (see Figure 1). The RNS1, RNS2 and RNS3 genes encode transcripts of $1.1,1.3$, and $1.1 \mathrm{~kb}$, respectively; of the three genes, RNS 2 is the most highly expressed in whole plants. The identification of the RNS genes in a self-compatible species suggests that this class of RNases is of general significance in RNA catabolism in higher plants. (Supported in part by a grant from the McKnight Foundation.) 


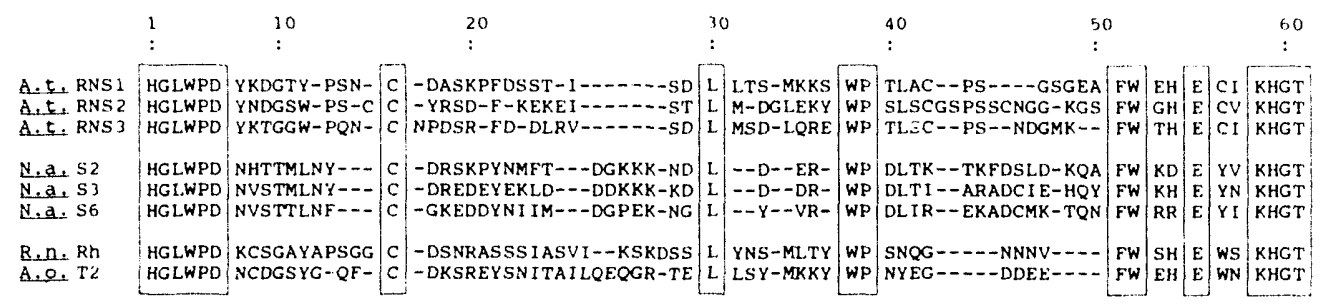

Figure 1. Sequence alignment of the RNS gene products with fungal and SRNases. Alignment of deduced amino acid sequences of the $A$. thaliana RNS genes with the S-RNases of $N$. alata (McClure et al., 1989), RNase $T_{2}$ of $A$. oryzae (Kawata et al., 1988) and RNase Rh of R. niveus (Horiuchi et al., 1988). Sequence alignment was based on that of McClure et al. (1988). Residues that are identical in all of the RNases are boxed. The sequences are numbered beginning with 1 for the first amino acid in the cioned fragment of RNS 1 . Dashes indicate gaps introduced to optimize the alignment. The abbreviations to the left of the figure are as follows: A.t., A. thaliana; N.a., N. alata; R.n., R. niveus; A.o., A. oryzae.

\section{References}

An, G. Plant Physiol. 79:568 (1985).

Fang, R-X., F. Nagy, S. Sivasubramaniam and N-H.Chua The Plant Cell 1:141 (1989).

Horiuchi, H., K. Yanai, M. Takagi, K. Yano, E. Wakabayashi E., A. Sanda, S. Mine, K. Ohgi and M. Irie. J. Blochem, (Tokyo) 103:408 (1988).

Kawata, Y., F. Sakiyama and H. Tamaoki. Eur. J. Biochem. 176:683. (1988).

Lindsten, T., C.H. June, J.A. Ledbetter, G. Stella and C.B. Thompson. Science 244:339 (1989).

McClure, B.A., V. Haring, P.R. Ebert, M.A. Anderson and A.E. Clarke. Nature 342:955 (1989).

Nagata, T., K. Okada, I. Takebe and C. Matsui. Mol. Gen. Genet. 184:161 (1981). Nagy, F., S.A. Kay and N-H. Chua. Genes Dev. 2:376 (1988).

Schuler, G.D. and M.D. Cole. Cell 55:1115 (1988).

Shaw, G. and R. Kamen. Cell 46:659 (1986).

Taylor C.B. and P.J. Green. Plant Physiol. In press (1991). 


\title{
RESISTANCE OF PLANTS TO ENVIRONMENTAL STRESS
}

\author{
Andrew D. Hanson. Pierre Lafontaine, Kent F. McCue, Jean Rivoal and Bala \\ Rathinasabapathi
}

In environments where stresses such as drought, salinity or flooding severely depress the growth of crop plants, the native vegetation may show high productivity (Boyer, 1982). This indicates that there is considerable scope for improving crop adaptation to unfavorable environments. However, the mechanisms responsible for adaptation to stresses are poorly understood at the genetic and biochemical levels (Boyer, 1982; Hanson et al., 1986). This lack of basic information is a barrier to both conventional plant-breeding and recombinant DNA (rDNA) approaches to improving stress resistance. By making it possible to transfer DNA across species boundaries, rDNA technology has greatly expanded the pool of stress resistance genes that might be used for crop improvement. The problem is to identify these genes. This research group is using a biochemical strategy to do so. First, metabolic pathways which may contribute to stress adaptation are sought. Second, the key enzymes of these pathways are found, and the corresponding genes isolated. Third, these genes are manipulated in order to evaluate their impact on plant stress resistance.

We are investigating two types of metabolic adaptations to stress: the accumulation of glycine betaine and other quaternary ammonium compounds (QACs) induced by salt- or water-stress; and lactate glycolysis induced by hypoxia. QACs can act as non-toxic cytoplasmic osmolytes, and hence permit metabolic function at low water potential (McCue and Hanson, 1990). Lactate glycolysis complements ethanol glycolysis during $\mathrm{O}_{2}$ deficit in roots and seeds of many plants (Roberts et al., 1984), and may be critical to survival in flooded soils.

\section{Expression of betaine aldehyde dehydrogenase from sugar beet}

Betaine aldehyde dehydrogenase $(B A D H)$ catalyzes the last step in betaine synthesis in plants (Weretilnyk et al., 1989). In sugar beet, the activity of BADH was found to increase two- to four-fold in both leaves and roots as the $\mathrm{NaCl}$ level in the irrigation solution was raised from 0 to $500 \mathrm{mM}$. The increase in enzyme activity was paralleled by an increase in the level of translatable BADH mRNA. To further investigate this stress induction, several CDNAs encoding BADH were cloned from a lambda gt10 library prepared from leaves of salinized sugar beet plants, by hybridization with a spinach BADH cDNA (Weretilnyk and Hanson, 1990). Three nearly full-length BADH cDNA clones were obtained, one of which was used as a probe in RNA gel blot analyses of total RNA. These analyses showed that salinization to $500 \mathrm{mM} \mathrm{NaCl}$ increased BADH mRNA levels four-fold in leaves and three-fold in the taproot. The stress-induction of BADH in sugar beet root tissues was unexpected, because in-vivo radiotracer labeling experiments had indicated that betaine synthesis takes place mainly if not solely 
In the leaves (Hanson and Wyse, 1982). (Supported in part, by grants from USDA-CRGO, and the BSDF.)

\section{Determination of choline-O-sulfaie by fast atom bombardment mass spectrometry}

Choline-O-sulfate occurs widely among marine algae and marine fungi (Blunden and Gordon, 1986; Catalfomo et al., 1972) and has been reported as a significant metabolite of ${ }^{35} \mathrm{SO}_{4}$ in higher plants (Wyn Jones and Storey, 1981). It has been suggested that it functions as a sulfur storage compound, as a detoxification product of sulfate anion, and as a compatible cytoplasmic solute. Levels of choline-O-sulfate have not been measured in higher plants. Accordingly, a sensittve and specific method was developed for determining choline-O-sulfate by fast atom bombardment mass spectrometry, using a deuterium labeled (do) choline-O-sulfate internal standard. This method was used to survey thirty native halophytic species from fourteen familles, collected from temperate and subtropical habitats. Twenty three species had very little choline-O-sulfate $(<2$ $\mu \mathrm{mol} \mathrm{g}^{-1} \mathrm{dry} \mathrm{wt}$ ) but Avicennia germinans contained $8 \mu \mathrm{mol} \mathrm{g}^{-1} \mathrm{dry} w \mathrm{w}$, and all six members of the family Plumbaginaceae tested (Limonium and Armeria species) accumulated $>100 \mu \mathrm{mol} \mathrm{g}^{-1}$ dry wt. The identity of choline-O-sulfate was confirmed by high resolution and tandem mass spectrometry, by ${ }^{1} \mathrm{H}$ nuclear magnetic resonance, and by analysis of hydrolysis products.

Synthesis and accumulation of quaternary ammonium compounds in Limonium spp. In response to salt stress

Fast atom bombardment mass spectrometry (Rhodes et al., 1987) was used to determine QACs in twenty two species from the widely distributed halophytic genus Limonium (Plumbaginaceae). Under saline conditions, all species accumulated choline-O-sulfate to high levels (40-150 $\mu \mathrm{mol} \mathrm{g}^{-1} \mathrm{dry}$ wt). Leaves of three species accumulated glycine betaine ( $>100 \mu \mathrm{mol} \mathrm{g}^{-1} \mathrm{dry} w$ ) but contained no detectable $B$-alanine betaine; the other species accumulated $B$-alanine betaine (40-150 $\mu \mathrm{mol} \mathrm{g}^{-1} \mathrm{dry} \mathrm{wt}$ ) but had little or no glycine betaine.

$$
\begin{aligned}
& \left(\mathrm{CH}_{3}\right)_{3} \cdot \stackrel{\oplus}{\mathrm{N}}-\mathrm{CH}_{2}-\mathrm{COO} \Theta \quad \text { Glycine betaine } \\
& \left(\mathrm{CH}_{3}\right)_{3} \cdot \stackrel{\oplus}{\mathrm{N}}-\mathrm{CH}_{2} \cdot \mathrm{CH}_{2}-\mathrm{COO}{ }^{\ominus} \quad \beta \text {-Alanine betaine } \\
& \left(\mathrm{CH}_{3}\right)_{3}-\stackrel{\oplus}{\mathrm{N}}-\mathrm{CH}_{2}-\mathrm{CH}_{2}-\mathrm{O}-\stackrel{\mathrm{O}}{\mathrm{O}}-\mathrm{O} \Theta \text { Choline-O-sulfate }
\end{aligned}
$$

Fig. 1. Quaternary ammonium compounds found in Limonium species. 
Leaf disks of both B-alanine betaine- and glycine betaine-accumulating species metabolized $\left[{ }^{14} \mathrm{C}\right.$ ]choline to choline-O-sulfate, but only the latter formed $\left[{ }^{14} \mathrm{C}\right]$ glycine betaine. Conversely, only B-alanine betaine-accumulators converted $B-\Gamma^{14} \mathrm{C}$ ]alanine to $B$-alanine betaine. When B-alanine betaine- and glycine betaineaccumulators were salinized with various levels of $\mathrm{NaCl}$, the levels of their respective betaines, plus that of choline-O-sulfate, were closely correlated with leaf solute potential. Sulfate salinization resulted in an increase in choline. 0 sulfate level and a matching decrease in glycine betaine level. These data establish that $B$-alanine betaine replaces glycine betaine, the common angiosperm betaine, in some but not all members of the genus Limonium, and indicate that both compounds as well as choline-O-sulfate may serve as compatible cytoplasmic csmolytes in this taxon. The data further suggest that the synthesis of choline-O-sulfate and glycine betaine may compete for choline. Such competition could have favored the evolution of the biosynthetic pathway for Balanine betaine.

\section{Osmoprotection of Escherichia coli and Salmonella typhimurium by choline-O- sulfate and B-alanine betaine}

The compatibility of a solute is determined by its chemical structure and is largely independent of the nature of the organism (Somero, 1986), so that bioassays of stimulatory effects on the growth of bacteria in high-osmolality media (osmoprotection) provide a general measure of compatibility (Csonka, 1989). Although choline-O-sulfate and B-alanine betaine can on physico-chemical grounds be reascinably assumed to be compatible solutes (Wyn Jones, 1984), there is little experimental evidence for this. The osmoprotective effects of these compounds were therefore evaluated in $E$. coli and $S$. typhimurium. When added at $1 \mathrm{mM}$ to medium containing $0.6 \mathrm{M} \mathrm{NaCl}, B$-alanine betaine and choline-Osulfate acted as osmoprotectants; both compounds were comparable in effectiveness to glycine betaine (Fig. 2). All three QACs decreased the doubling time to approximately 1.5 hours, although the lag phases for B-alanine betaine and choline-O-sulfate were consistently longer than for glycine betaine.

Neither of the bacterial strains used is able to oxidize choline to glycine betaine and, as expected, neither was protected by choline so that the effect of cholineO-sulfate cannot be attributed to the cleavage of the ester bond followed by oxidation to glycine betaine. This was confirmed by using ${ }^{14} \mathrm{C}$-labeled choline-Osulfate; $>99 \%$ of the label accumulated in the bacterial cells was in the form of choline-O-sulfate. 


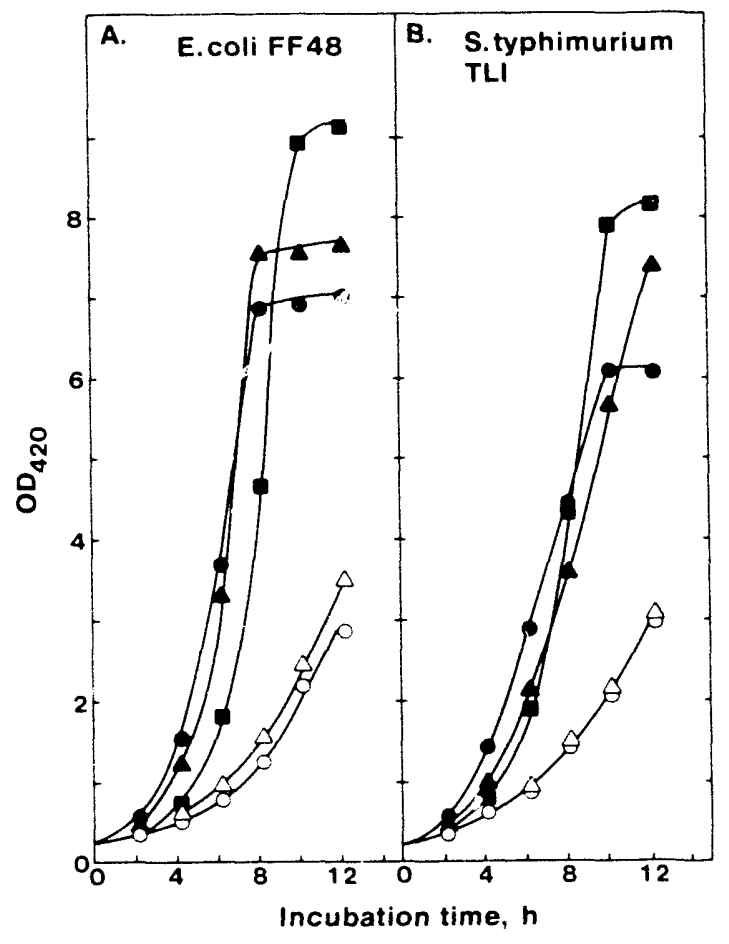

Fig. 2. Osmoprotection by B-alanine betaine and choline-O-sulfate. E. coli mutant FF48 (betA, unable to convert choline to betaine) and $S$. typhimurium wild type were grown aerobically at $37^{\circ} \mathrm{C}$ in minimal medium containing $0.6 \mathrm{M} \mathrm{NaCl}$ alone $(0)$, or with $1 \mathrm{mM}$ choline-O-suliate ( 4$)$, B-alanine betaine $(-)$, choline $(\Delta)$, or glycine betaine (0).

\section{Lactate production and secretion by roots of Limonium sinuatum}

Although hypoxia induces isozymes of L-lactate dehydrogenase (LDH) in the roots of many plants, experimental measurements with mesophytes subjected to anoxia indicate that the flux to lactate is generally far less than that to ethanol (e.g. Hoffman et al.. 1986). Because the roots of many halophytes penetrate soil horizons that are completely anoxic, the role of lactate fermentation was investigated in the sali marsh species Limonium sinuatum. Roots were incubated for $8 \mathrm{~h}$ under anoxic conditions in the presence of $\left[U-{ }^{14} \mathrm{C}\right]$ glucose, and labeled metabolites were analyzed by distillation, ion exchange chromatography and TLC. With roots previously acclimated to hypoxia, lactate was always a significant product; the ratio of labeled lactate/ethand ranged from approximately 0.3 to 2.0 in various experiments. In all cases, most of the labeled lactate was found in the medium. These results suggest a parallel with fermentative bacteria, vich can use lactate secretion to generate the transmembrane electrochemical proton gradient that is the driving force for transport of ions and other solutes (Konings, 1985). 


\section{References}

Blunden, G. and S.M. Gordon. Prog. Phycol. Res. 4:39 (1986).

Boyer, J.S. Science 218:443 (1982).

Catalfomo, P., J.H. Block, G.H. Constantine and P.W. Kirk. Marine Chem. 1:157 (1973).

Csonka, L.N. Microbiol. Rev. 53:121 (1989).

Hanson, A.D., N.E. Hoffman and C. Samper. HortScience 21:1313 (1986).

Hanson, A.D. and R. Wyse. Plant Physiol. 70:1191 (1982).

Hoffman, N.E., A.F. Bent and A.D. Hanson. Plant Physiol. 82:658 (1986).

Konings, W.N. Trends Biochem. Sci. 10:317 (1985).

McCue, K.F. and A.D. Hanson. Trends Biotech. 8:358 (1990)

Rhodes, D., P.J. Rich, A.C. Myers, C.R. Reuter and G.C. Jamieson. Plant Physiol. 84:781 (1987).

Roberts, J.K.M., J. Callis, O. Jardetzky, V. Walbot and M. Freeling. Proc. Natl. Acad. Sci. USA 81:6029 (1984).

Somero, G.N. Am. J. Physiol. 251:R197 (1986).

Weretilnyk, E.A., S. Bednarek, K.F. McCue, D. Rhodes and A.D. Hanson. Planta 178:342 (1989).

Weretilnyk, E.A. and A.D. Hanson. Proc. Natl. Acad. Sci. USA 87:2745 (1990). Wyn Jones, R.G. Recent Adv. Phytochem. 18:55 (1984).

Wyn Jones, R.G. and R. Storey. In: The Physiology and Biochemistry of Drought Resistance (L.G. Paleg and D. Aspinall, eds.), pp. 171-204, Academic Press, N.Y. (1981). 


\title{
STUDIES ON HORMONE BIOSYNTHESIS AND ACTION
}

\author{
Hans Kende. Leonard Edelman, Susanne Hoffmann, David Olson, Scott Peck, \\ Margret Sauter, Joseph White, Ronda White and Renate de Zacks
}

During the past year, we have continued work on ethylene biosynthesis and action and have resumed research on cytokinin action. Since the discoverey that 1-aminocyclopropane-1-carboxylic acid (ACC) is the immediate precursor of ethylene in higher plants (Adams and Yang, 1979) ethylene formation has been investigated intensively at both the physiological and biochemical levels. Research in our laboratory focused mainly on the enzymes of this pathway. Important contributions were the first description of ACC synthase by Boller et al. (1979), the purification of this enzyme using monoclonal antibodies (Bleecker et al., 1986) and the discovery that the ethylene-forming enzyme (EFE) is localized, at least in part, in the plant vacuole (Guy and Kende, 1984). We have now entered a new phase in ethylene research. Last year, the first nucleotide sequence of a gene encoding ACC synthase was published (Van Der Straeten et al., 1990). Below, we report on the differential expression of two $A C C$ synthase genes in pericarp tissue of ripening tomato fruits. We also describe mutants of Arabidopsis thaliana that are impaired in ethylene-forming capacity. Research on ethylene action is also progressing rapidly. Work with ethylene-insensitive Arabidopsis mutants begun in this laboratory (Bleecker et al., 1988) promises to yield information on individual steps of the transduction pathway. Our research on ethylene action deals with the effect of this hormone on growth of deepwater rice. Recent results indicate that ethylene may stimulate internodal elongation by shifting the ratio of gibberellin (GA), a growth promoter, and abscisic acid (ABA), a growth inhibitor, in favor of the former.

While our knowledge of ethylene biosynthesis and action has advanced remarkably, progress in elucidating the mechanism of action of cytokinins has been generally slow. Twenty years ago, we found that cytokinins enhance the activity of nitrate reductase in Agrostemma githago independently of nitrate which serves usually as inducer of this enzyme (Kende et al., 1971). Our results indicated that cytokinins and nitrate act in distinct fashion. We have resumed work on this problem and, using CDNA probes, are trying to ascertain whether cytokinins and nitrate regulate the same or two different genes encoding nitrate reductase.

\section{Ethylene Biosynthesis}

Ethylene is involved in the regulation of many developmental and stress-induced processes in plants. It is synthesized from L-methionine via S-adenosyl-Lmethionine and ACC. The rate-limiting enzyme of this pathway is, in most instances, ACC synthase, which was first identified in homogenates of tomato pericarp tissue (for a review see Kende, 1989). A variety of chemical and 
environmental factors, e.g. indole-3-acetic acid, noxious chemicals, physical wounding and chilling, stimulate ethylene formation by enhancing the activity of ACC synthase. One of the important problems regarding the regulation of ethylene biosyn! iesis concerns the question of whether diverse stimuli control the expression of one ACC-synthase gene or whether there are multiple ACCsynthase genes, each of which is regulated by a specific class of stimuli. A systematic analysis of enzyme preparations from wound-induced tomato pericarp tissue by SDS/PAGE and isoelectric focusing did not show size or charge variants of ACC synthase (White and Kende, 1990). However, sequencing of a tryptic fragment containing the active site of ACC synthase fron, wound-induced, ripening tomato fruits provided evidence for the existence of two isoforms which differed, in this particular peptide, by one amino acid (Yip et al., 1990). This indicated that tomato fruits contain at least two isoforms of ACC synthase which could not be distinguished by biochemical or immunological means. Recently, Van Der Straeten et al. (1990) published the first full-length sequence of a cDNA encoding ACC synthase from tomatoes. These same authors also isolated a 420bp partial ACC-synthase CDNA clone that varied in its nucleotide sequence from the first one, which indicated again that tomato fruits contain more than one isoform of the enzyme.

\section{Differential expression of two ACC-synthase genes in tomato fruits}

ACC synthasc was purified by immunoadsorption and subjected to $\mathrm{CNBr}$ fragmentation. Partial amino acid sequences were obtained from three cleavage peptides. All three amino acid sequences were contained in the full-length clone of Van Der Straeten et al. (1990). Based on our amino acid sequences derived from isolated ACC-synthase protein and the published nucleotide sequences of Van Der Straeten et al. (1990), we prepared oligonucleotide primers and amplified a 288-bp fragment from tomato-fruit CDNA using the polymerase chain reaction (PCR). This PCR product was cloned into pUC19 and used to screen a CDNA library prepared from poly $(A)^{+}$RNA of ripening tomato fruits. Three clones were isolated which differed from the full-length clone of Van Der Straeten et al. (subsequently referred to as ACC-synthase 1) but which contained the nucleotide sequence of the partial clone of Van Der Straeten et al. (subsequently referred to as ACC-synthase 2). The clone encoding ACC synthase-2 was found to be 1652 bp long. This included 48 bp of the $5^{\prime}$ untranslated region and the poly-A tail. The overall DNA sequence similarity between the coding regions of ACC-synthase 1 and 2 is $75 \%$; the deduced amino acid sequences are $68 \%$ homologous at optimal alignment. Since the 3 ' ends of the two clones are dissimilar, gene. specific probes were amplified by PCR in order to compare the expression 'or the two genes. Poly $(A)^{+}$RNA was prepared from tomato pericarp tissue at four different stages of ripening, either immediately after isolation of thia tissue or after $4 \mathrm{~h}$ of wounding. RNA blot analysis (Fig. 1) showed that the level of mRNA encoding ACC-synthase 1 increased during ripening and as a result of wounding. The level of ACC-synthase 2 mRNA increased during ripening but was little or not 
at all affected by wound stress. Further analysis using RNA from pink fruits wounded for up to $8 \mathrm{~h}$ showed that the level of mRNA for ACC-synthase 1 greatly increased within $40 \mathrm{~min}$, while that for ACC synthase-2 remained constant even after $\mathbf{8} \mathrm{h}$ of wounding. Gene-specific probes hybridized to fragments of different sizes in Southern blots, confirming that the two ACC-synthase isoforms are encoded by different genes.

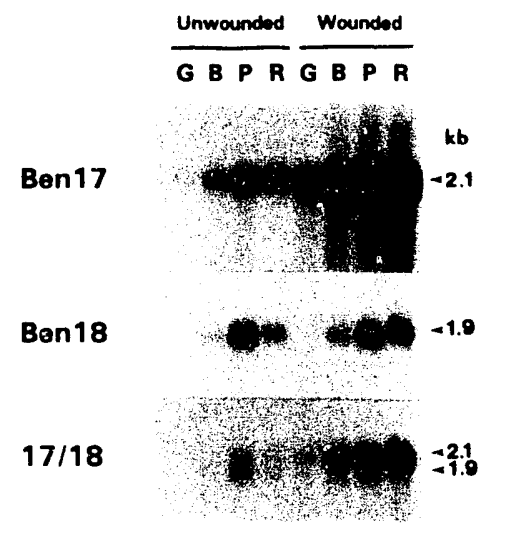

Figure 1. Differential expression of the genes encoding ACC-synthase 1 and 2. Poly $(A)^{+}$RNA isolated from pericarp tissue of mature green $(G)$, breaker $(B)$, pink $(P)$ and light-red $(R)$ tomato fruits at time 0 (unwounded) or $4 \mathrm{~h}$ after wounding was separated by electrophoresis on agarose gels and blotted onto a nylon membrane. The filters were probed with ${ }^{32}$ P-labeled Ben17, the gene-specific probe for ACC-synthase 1 (top); with ${ }^{32} \mathrm{P}$-labeled Ben 18, the gene-specific probe for ACC-synthase 2 (middle); and with a mixed probe consisting of ${ }^{32} \mathrm{P}$-labeled Ben17 and Ben18.

\section{Screening for ethylene biosynthetic mutants in Arabidopsis thaliana}

Mutants defective in ethylene formation would be very useful for studying ethylene biosynthesis and action. We have been screening $\mathrm{M}_{2}$ populations of Arabidopsis with the aim of finding mutants deficient either in the activity of ACC-synthase or of the ethylene-forming enzyme (EFE). Although not yet characterized biochemically, the latter is assumed to be an oxidase that converts ACC to ethylene. To date, approximately $125,000 \mathrm{M}$ plants have been examined. The ethylene-producing capacity of individual plants was assessed according to the following procedure. A mature leaf was removed from the plant and enclosed in a vial containing some water. After 2 and $5 \mathrm{~h}$, the level of ethylene in the head space of each vial was determined by gas-chromatography. In order to increase the number of putative mutants among the population of plants tested for ethylene-synthesizing capacity, a pre-selection scheme was employed. Since ethylene mediates closure of the plumular hook in etiolated dicotyledonous 
seedlings, a plant that produces less ethylene or is insensitive to it will have at least a partially open hook. Etiolated Arabidopsis seedlings displaying partly to fully opened hooks were considered putative ethylene biosynthetic mutants and were allowed to grow to maturity. To select mutants specifically impaired in EFE, ACC was included in the germination medium. Under these conditions, mutants blocked at or prior to the ACC-synthase step would maintain a closed hook. Seedlings that are grown on ACC and still have open hooks would include ethylene-insensitive mutants as well as mutants blocked in EFE. Fully grown plants that had an open apical hook as seedlings were tested for ethylene-forming capacity as described above. Mutants that consistently produced low amounts of ethylene have now been tested through the $M_{4}$ or $M_{5}$ generation, and backcrossing to the wild-type parent has been started to remove unlinked mutations. When we began the search for ethylene biosynthetic mutants, we anticipated finding null mutants in ACC synthase and possibly in EFE. However, it has become clear from recent work that ACC synthase has multiple forms (see above), which appears to be the case in Arabidopsis as well (Theologis, personal communication). Since null mutations in ACC-synthase activity (and possibly in EFE activity as well) are not likely to exist, pairwise crosses between the mutants have been started to define complementation groups and to construct double mutants that may lack ethylene-forming capacity. (Supported, in part, by USDA Grant No. 89-37261-4461.)

\section{Regulation of Growth in Deepwater Rice}

We are studying the role of ethylene and other plant hormones in the regulation of growth in deepwater rice, which has the capacity to elongate rapidly when submerged. This adaptive feature permits deepwater rice to survive prolonged flooding during the monsoon season by maintaining part of its foliage above the rising water. The environmental signal for growth is the lowered level of oxygen within the submerged tissue. Low oxygen tensions enhance the activity of ACC synthase and, thereby, promote ethylene synthesis. Plants kept either under low oxygen tensions or in air containing ethylene elongate almost as rapidly as submerged plants do. Ethylene does not stimulate growth directly but, rather, increases the plant's sensitivity to gibberellin (GA; for a review see Kende, 1987). One of the problems under current investigation concerns the mechanism by which ethylene enhances the growth-promoting activity of GA.

The growth response of deepwater rice is based, at least in part, on increased cell-wall extensibility in the growing region of the internode (Kutschera and Kende, 1988). Therefore, we are also examining the difference in cell wall composition between rapidly and slowly elongating internodes. We are analyzing biochemical events in the cell wall that may be related to either promotion of rapid growth or to irreversible cessation of growth. (Supported, in part, by NSF Grant No. DCB8718873.) 
The role of abscisic acid (ABA) in the growth response of deepwater rice

Treatment with ethylene reduces the level of ABA in some plants (Zeevaart, 1883). Since $A B A$ is a growth inhibitor, we examined the possibility that increased responsiveness to GA is due to reduced levels of $A B A$ in the tissue. In other words, enhanced growth may be the consequence of a shift in the ratio between a growth inhibitor and a growth promoter. Results obtained with rice seedlings are consistent with this hypothesis (Plant Research 1989). Fluridone, an inhibitor of carotenoid and, hence, ABA biosynthesis, reduced the level of ABA in coleoptiles and nearly doubled their growth rate. Since green tissue contains ample amounts of carotenoids to serve as precursors for $A B A$ formation, the effect of fluridone on growth cannot be assessed with mature deepwater rice plants. Because of this, we tested our hypothesis by examining the effect of applied ABA and GA on growth and by moasuring the level of $A B A$ in submerged and ethylene-treated plants.

Applied ABA inhibited submergence-induced internodal growth of deepwater rice, and added gibberellic acid (GAs) fully overcame this inhibition. In terms of applied growth regulators, the balance of $A B A$ and $G A$ did, therefore, determine the growth rate of deepwater-rice plants. Next, we examined the effect of submergence and ethylene treatment on ABA content of internodes. Growth was induced by submerging whole plants in 300 -liter tanks or by enclosing them in plastic tubes through which air containing 3-5 $\mu \mathrm{l} / \mathrm{l}$ ethylene was circulated. Airgrown plants served as control. ABA was extracted from the growing zone of the uppermost internode, and its concentration was determined using an immunoassay. Both, submergence and treatment with ethylene, reduced $A B A$ content in internodal tissue within $3 \mathrm{~h}$ to about one-fourth of its original level (Figs. 2, 3). Therefore, submergence and ethylene may indeed promote growth, at least in part, by reducing the concentration of $A B A$ in the elongating region of the internode.

Figure 2. Abscisic acid levels in the elongation zone of the top-most internodes of air-grown and submerged deepwater-rice plants. The bars denote standard error. 


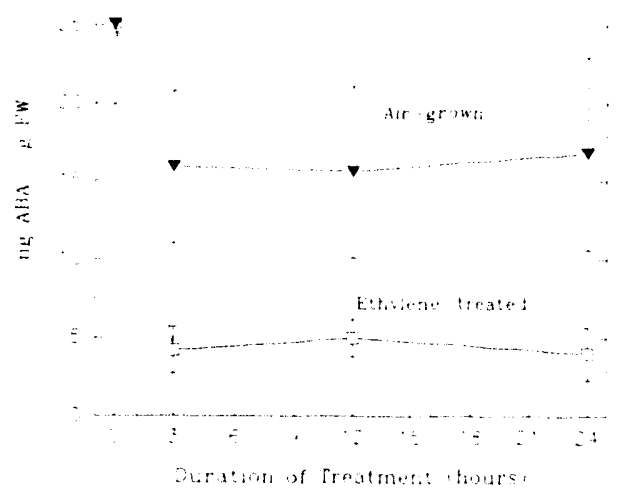

Figure 3. Abscisic acid levels in the elongation zone of the top-most internode of air-grown and ethylene-treated deepwater-rice plants. The bars denote standard error. Analysis of variance showed that the effect of ethylene was highly significant $(p<0.005)$.

\section{Cell wall analysis in deepwater rice}

Rapid growth in deepwater rice very likely requires suppression of processes that restrict elongation and promotion of processes that support it. We examined lignification as one of the cellular events that limit growth irreversibly, and changes in the hemicellulosic composition of the cell wall that appear to be required for sustained cell elongation. We showed previously that the activities of two enzymes of the lignin biosynthetic pathway, phenylalanine ammmonia-lyase and coniferylalcohol dehydrogenase, were much lower in the newly formed internodal tissue of submerged or $\mathrm{GA}_{3}$-treated plants than in control internodes (Plant Research 1989). These reduced enzyme activities were reflected in the lignification of the respective tissues. The lignin content per dry weight of cell wall material was five- to sixfold lower in the elongating part of submerged internodes than in the corresponding region of air-grown control internodes. Submergence did not affect the lignin content in the older, preexisting portions of the stem. Microscopic analysis showed that lignification was limited to certain cells or cell layers. In the intercalary meristem (IM) of air-grown plants, only the annular rings of the protoxylem were lignified. In the tissue above the IM, the xylem elements, sclerenchyma cells surrounding the xylem vessels and subepidermal sclerenchyma cells showed strong lignin staining. In the IM of submerged plants, the annular rings of the protoxylem were lignified just as in control plants, but in the newly formed tissue above the IM, very little lignification of subepidermal cells and of the cells surrounding the xylem was observed. No difference could be detected in the intensity of lignin staining in the oldest region uf internodes from air-grown and submerged plants. These results demonstrate that suppression of lignification is one component of the growth process in deepwater rice. 
B-Glucan, an unbranched homopolymer consisting of (1-3)-B-D- and (1-4)-B-Dglucosyl units, is a unique component of the cell wall of graminaceous monocots. It may comprise up to $30 \%$ of the hemicellulose fraction which, in turn, is at least $50 \%$ of the total cell wall material of this group of plants. Since the synthesis of hemicelluloses has been implicated in the control of cell growth (Carpita, 1987), we set out to determine the pattern of B-glucan distribution in rapidly growing and in control rice internodes. We found that induction of cell elongation is correlated with a marked increase in the level of B-glucan in the elongation zone. Therefore, the increase in B-glucan levels is being studied as a process that is positively linked to growth.

\section{Regulation of Nitrate Reductase by Cytokinins}

We are studying the regulation of nitrate reductase (NR) by cytokinins and nitrate in embryos of Agrostemma githago. While cytokinins enhance NR activity in several plants in the presence of nitrate, only in Agrostemma have cytokinins been shown to do so in the absence of nitrate. This and the fact that induction of NR activity by nitrate and cytokinins is additive indicate that the two effectors act independently of each other. Very little is known about how cytokinins exert their effects in higher plants. We shall use the Agrostemma system to study the regulation of gene expression by cytokinins.

First, we optimized the experimental conditions for the induction of NR with regard to incubation conditions and the concentrations of cytokinins and nitrate. We used mostly benzyladenine (BA), a synthetic cytokinin, but the natural cytokinin zeatin elicited the same response. Since light had been shown to enhance the induction of NR by nitrate and BA in several plants, we examined the effect of illumination on the development of NR in embryos of Agrostemma. We did not find any interaction between light and either nitrate or BA on the induction of NR. Once the experimental conditions had been defined, we set out to determine whether or not $B A$ and nitrate regulate the same or different genes encoding NR. We used a partial cDNA clone for NR from tobacco (BMC102010; Calza et al., 1987) to probe genomic DNA of Agrostemma for related or homologous sequences. Southern blot analysis showed three EcoR1 and three Hindlll bands ranging from 6.2 to $16 \mathrm{~kb}$ to which BMC102010 hybridized. The NR genes cloned thus far range from 5.3 to $6.1 \mathrm{~kb}$. Therefore, each of the crosshybridizing bands could contain a NR gene. This indicates, but does not prove, that Agrostemma may have two to three NR genes.

Poly $(\mathrm{A})^{+}$RNA was isolated from embryos induced with nitrate, $\mathrm{BA}$, and nitrate plus BA. Blot analysis of this RNA from induced embryos showed a $3.5-\mathrm{kb}$ band cross-hybridizing to BMC102010. No NR mRNA was detected in the RNA preparation from control embryos. We determined the relative amounts of NR mRNA present under each of the inductive conditions by densitometry of the autoradiographs (Fig. 4) and found that the effects of BA and nitrate at optimal 
concentrations were strictly additive, just as had been observed for the induction of enzyme activity. These results indicate that the regulation of NR by nitrate and $B A$ may be at the transcriptional level.

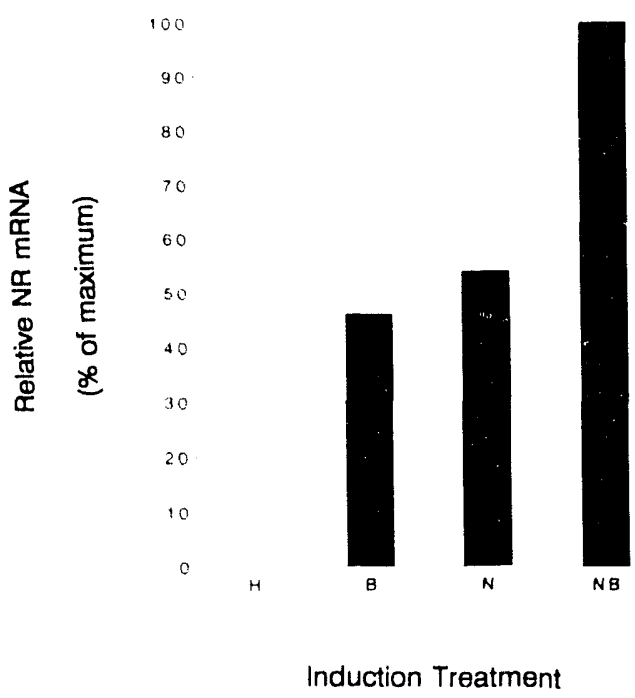

Figure 4. Relative levels of NR mRNA in Agrostemma githago. Excised embryos were incubated for $6 \mathrm{~h}$ in water $(H), 1 \mu \mathrm{MBA}(B), 50 \mathrm{mM}$ nitrate $(\mathrm{N})$, or $50 \mathrm{mM}$ nitrate plus $1 \mu \mathrm{MBA}$ (NB). Poly(A) ${ }^{+}$RNA was separated by electrophoresis on an agarose gel, transferred to a nylon membrane, and hybridized to the BMC102010 probe. The mRNA levels were quantified by densitometry of the autoradiograph.

\section{References}

Adams, D.O. and S.F. Yang. Proc. Natl. Acad. Sci. USA 76:170 (1979).

Boller, T., R.C. Herner and H. Kende. Planta 145:293 (1979).

Bleecker, A.B., W.H. Kenyon, S.C. Somerville and H. Kende. Proc. Natl. Acad. Sci. USA 83:7755 (1986).

Bleecker, A.B., M.A. Estelle, C. Somerville and H. Kende. Science 241:1086 (1988).

Calza, R., E. Huttner, M. Vincentz, P. Rouze, F. Galangau, H. Vacheret, I. Cherel, C. Meyer, J. Kronenberger and M. Caboche. Mol. Gen. Genet. 207:552 (1987).

Carpita, N.C. In: Physiology of Cell rxpansion during Plant Growth (D.J.

Cosgrove and D.P. Knievel, eds.), pp. 28-45, Am. Soc. Plant Physiol., Rockville (1987).

Guy, M. and H. Kende. Planta 160:281 (1984).

Kende, H., H. Hahn and S. Kays. Plant Physiol. 48:702 (1971).

Kende, H. In: Physiology of Cell Expansion during Plant Growth (D.J. Cosgrove and D.P. Knievel, eds.), pp. 227-238, Am. Soc. Plant Physiol., Rockville (1987). Kende, H. Plant Physiol. $91: 1$ (1989).

Kutschera, U. and J. Kende. Plant Physiol. 88:361 (1988). 
Van Der Straeten, D., L. Van Wiemeersch, H.M. Goodman and M. Van Montagu. Proc. Natl. Acad. Sci. USA 87:4859 (1990).

White, J. A. and H. Kende. J. Plant Physiol. 136:646 (1990).

Yip, W.-K., J.-G. Dong, J.W. Kenny, G.A. Thompson and S.F. Yang. Proc. Natl. Acad. Sci. USA 87:7930 (1990).

Zeevaart, J.A.D. Plant Physiol. $71: 477$ (1983). 


\section{PLANT CELL WALL PROTEINS}

Derek T.A Lamport. Hasan Alizadeh, Lawrence Benbow, Renate de Zacks, Cynthia Fong, Marcia J. Kieliszewski, Wu Qi, Linda Schnabelrauch and Brad Upham

We view the primary cell wall as an extracellular, pleiomorphic organelle possessing structural integrity, enzymic autonomy and sufficient information for self-assembly as an interpenetrating-network (IPN) micro-composite. If, as seems likely, cell extension depends on a regulated "biochemical creep," then we need to identify the IPNs and their contribution to wall rheology. The "warp-weft" hypothesis, which suggests that a cellulose "warp" is mechanically coupled by an extensin "weft", is a step towards the ultimate goal of a complete primary cell wall model.

Most of our work utilizes cell suspension cultures as a source of primary cell walls, not merely for historical reasons, but because cell cultures arguably represent a primitive "algal" state producing a "generic" cell wall. This greatly facilitates comparison between species, especially of HRGPs (hydroxyproline-rich glycoproteins, including basic extensins and acidic arabinogalactan-proteins) directly elutable from the cell surface of growing cells (the intact cell elution technique). Thus HRGPs first described for cultured cells also occur in the whole plant.

However, extensins and arabinogalactan proteins are not the whole story. There are also enzymic wall proteins. In addition, many species produce walls with a low hydroxyproline-content yet still containing as much as $20 \%$ protein. Identification of a new structural protein in hydroxproline-poor cell walls is a promising new area.

\section{Histidine-rich extensins from Zea mays}

We used the "intact cell elution technique" to isolate a histidine-rich HRGP (HHRGP) from the surface of a Zea mays cell suspension culture (cv. Black Mexican). The HHRGP contained $65 \%$ carbohydrate occurring as arabinosylated Hyp (63 mole\% Ara), and galactose ( $3 i$ mole\%) possibly occurring as polygalactose on threonine or serine. Deglycosylated HHRGP migrated on SDSPAGE with an apparent $M$ of $70 \mathrm{kD}$, while glycosylated HHRGP did not migrate.

Antibodies raised against deglycosylated HHRGP were specific for deglycosylated HHRGP and deglycosylated dicot extensins, but showed virtually no crossreactivity with glycosylated extensins. Glycosylated HHRGP chromatographed as a single peak through three cation exchangers, gel permeation and reversed phase column chromatography, but resolved after HF deglycosylation into two bands $(M=68 \& 70 \mathrm{kD})$ on SDS-PAGE. To determine if the bands represented 
two significantly different proteins, rather than HHRGP isoforms or products of HF clipping of a specific peptide bond, we chromatographed deglycosylated HHRGP through a reversed phase column and analyzed the extreme leading and trailing edges of the obviously heterogeneous peak. Amino acid analyses of the peak edges were identical, within experimental error. We also rechromatographed glycosylated HHRGP through tandem analytical Superose-6 gel filtration columns. The HHRGP chromatographed as a single narrow symmetrical peak whose leading and trailing edges again had identical amino acid compositions. These results suggest that HF deglycosylation clips a specific HHRGP peptide bond.

The chymotryptic peptide map of the deglycosylated HHRGP gave 3 peptides showing significant sequence identity (66-88\%) with the $\mathrm{N}$-terminus of the Type 3 repetitive domain of dicot extensins, I.e., Ser-Hyp-Hyp-Hyp-Hyp-Ser-Hyp-SerHyp-Hyp-Hyp-Hyp-Tyr-Tyr-Tyr-Lys, thereby defining the HHRGP as a member of the extensin family as follows:

HC6: Ser-Hyp-Hyp-Hyp-His-Ser-Hyp-Ser-Hyp-Gly-

HC10: Ala-Hyp-Hyp-Hyp-His-Phe-Pro-Ser-Hyp-Hyp-Ala-Ala-

Asn-Ala-Ala-Hyp-Hyp-Hyp-Ala-His-Tyr

HC11: Ala-Hyp-Hyp-Hyp-His-Phe-Pro-Ser-Hyp-Hyp-Hyp(Thr)-His-His-HisHyp-Hyp-Hyp-Tyr

HC12: Ala-Ala-Hyp-Hyp-Hyp-His-Phe-Pro-Ser-Hyp-Hyp-

Thus, HHRGP is homologous with dicot Type 3 extensin repetitive domains, and therefore is the second member of the extensin family isolated from a monocot.

\section{Extensin phylogeny: the gymnosperm, Douglas fir}

Because of our interest in phylogeny, and as one part of a general strategy to define extensin functional domains, we set out to identify first the most highly conserved extensin motifs. Thus, we isolated HRGPs from the typical gymnosperm, Douglas fir. Douglas fir cultures yielded two HRGPs. The smaller of the two was similar in composition to a typical Type 1 extensin and remains to be characterized further. The larger monomer was deficient in serine; because it had a composition resembling the Repetitive Proline-Rich Proteins (RPRPs), we characterized it further by peptide mapping, sugar analysis, and a hydroxyproline glycosylation profile. The simple peptide map, indicating a periodic structure, was confirmed by a 45-residue partial sequence of the major chymotryptide: Lys-ProHyp-Val-Hyp-Val-Ilu-Pro-Pro-Hyp-Val-Val-Lys-Pro-Hyp-Hyp-Val-Tyr-Lys-Pro-HypVal-Hyp-Val-Ilu-Pro-Pro-Hyp-Val-Val-Lys-Pro-Hyp-Hyp-Val-Tyr-Lys-llu-Pro-Hyp-HypVal-llu-Lys-Pro..., which contained two instances of the 5-residue motif Hyp-HypVal-Tyr-Lys, and 5 instances of the general motifs X-Pro-Val-Tyr-Lys, Pro-Pro-Val$X$-Lys, and Pro-Pro-X-Tyr-Lys (where the Pro residues can be hydroxylated). It is therefore homologous with typical angiosperm RPRPs, and extensins from tobacco, tomato, beet, carrot, and petunia. Sugar analysis, confirmed by quantitative identification of hydroxyproline arabinosides, showed small but 
significant amounts of arabinose. This is the first isolation of a gymnosperm extensin and the first indication that some RPRPs are indeed glycosylated.

\section{Tomato prolyl hydroxylase}

Hydroxyproline comprises as much as $40 \%$ of HRGP amino acids, and is the major site of HRGP O-glycosylation (carbohydrate accounts for 25 - 95\% of HRGP dry weight). In both plants and animals, the conversion of proline to hydroxyproline is a posttranslational event catalyzed by the oxygenase, prolyl hydroxylase. Although animal prolyl hydroxylases are well characterized, prolyl hydroxylase trom higher plants has never been purified and has been only partially characterized using the artificial substrate, polyproline-ll. This has led to the dubious conclusion that a polyproline-ll conformation is required for enzyme activity. Our recent work with maize extensins implies that multiple forms of prolyl hydroxylase exist, and that a polyproline-II conformation is not a requirement for recognition; rather, specificity is dictated by the amino acid sequence. To test this hypothesis we are preparing "natural" polypeptide substrate for prolyl hydronylase by salt elution of the surface of suspension cultured cells treated with $\alpha, \alpha^{\prime}$-dipyridyl, an inhibitor of prolyl hydroxylase. We have thereby isolated a non-hydroxylated extensin monomer that is proline- and tyrosine-rich, and contains virtually no hydroxyproline. We will use this substrate to characterize prolyl hydroxylases from a variety of plant species (i.e., tomato, Douglas fir, maize). We have also synthesized peptide substrates with sequences based on naturally occurring extensin sequences (except that Pro replaces Hyp in the peptide sequences). One of our prolyl hydroxylase substrates, a synthetic peptide based on the major repeating sequence of our Douglas fir extensin, $V-V$ K-P-P-P-V-Y-K, was also serendipitously a substrate for a chymotrypsin-like cell wall protease discussed below.

\section{A cell wall protease?}

The cell surface of tomato cell suspension cultures yielded a salt eluate containing chymotryptic proteolytic activity, judging by assay with the synthetic peptide V-V-K-P-P-P-V-Y-K. The activity is heat labile, time- and substratedependent, and has a pH optimum of 7.4, although the enzyme is active from $\mathrm{pH}$ 3.7 to $\mathrm{pH}$ 9.5. However, a quantitative comparison of intracellular chymotryptic activity to extracellular (specifically, that in the wall) chymotryptic activity showed that intracellular activity is 16 times of the wall eluate, and cleaves the same bonds. Furthermore, the wall protease does not degrade any wall proteins that we provided as substrate (i.e., various extensins). Thus, the cell wall proteolytic activity may be due to cell breakage and subsequent adsorption to the wall rather than being a cell wall protease per se. 


\section{Extensin peroxidase and extensin crosslinks}

The "warp-weft" model of the primary cell wall hypothesizes that extensin monomers are intermolecularly crosslinked orthogonal to the cellulose microfibril, thus mechanically coupling the major load-bearing polymer: cellulose. This crosslinked extensin network is insoluble in anhydrous hydrogen fluoride (Mort and Lamport, 1977); however, extensin also occurs as a small pool of soluble flexuous rodlike monomers which are precursors to the network. Therefore, two questions of particular interest are:

1. How are the intermolecular crosslinks generated in muro?

2. What is the chemical identity of the intermolecular crosslink.

Everdeen et al. (1988) viemonstrated heat labile, peroxide-dependent crosslinking activity elutable from the cell surface of cultured tomato cells. Subsequent experiments showed that the crosslinking activity was mostly due to an acidic peruxidase (pi 4.6) present predominantly in the growth medium rather than in the cytosol. In control experimentr, we homogenized washed culiured tomato cells $(300 \mathrm{ml}, 50 \% \mathrm{pcv})$ in a French pressure cell and isoelectric-focused the soluble fraction. The cytosolic/vacuolar acidic peroxidase fractions showed no crossilinking activity.

The of 4.6 peroxidase from the growt? medium was partially purified by ultracentrifugation (to remove the pectin), DEAE-Trisacryl and HPLC-DEAE chromatography, resulting in a 90 -fold purification, $45 \%$ jield and an $R Z$ (Reinheitzahl) of 0.2. A second, minor, acidic peroxidase that was eluted from the HPLC-DEAE column had $25 \%$ of the crosslinking activity of the pl 4.6 peroxidase. Purified basic peroxidase (pl 9.0) had only $0.7 \%$ of the activity of the pl 4.6 peroxidase. The specific activity of the pl 4.6 peroxidase after HPLC-DEAE fractionation was $5473 \mathrm{mg}$ extensin crosslinked/min.mg peroxidase under standard assay conditions: $40 \mathrm{mM}$ citrate $/ \mathrm{pH} 6.0,3 \mathrm{mg} / \mathrm{ml}$ extensin monomer, $100-250 \mathrm{ng} / \mathrm{ml}$ peroxidase, $60 \mu \mathrm{MH}_{2} \mathrm{O}_{2}$, assay volunie $=20 \mu \mathrm{l}$, room temperature (i.e., an enzyme:substrate ratio varying from $1: 30,000 \mathrm{w} / \mathrm{w}$ to $1: 12,000$ ).

SDS-PAGE showed three major polypeptides in the active HPLC-DEAE fraction that ranged in molecular weight from 25 to $35 \mathrm{kD}$. An alternative purification scheme utilizing preparative isoelectric focusing after DEAE chromatography gave similar results on SDS-PAGE, suggesting that these three polypeptides have similar net charge as well as similar size.

The rates of crosslinking of tomato extensin I and soybean extensin were similar, but the rates for tomato extensin II, Ginkgo extensin II and carrot extensin were $40 \%$ and for sugarbeet was $2 \%$ of the rate of crosslinking of tomato extensin 1 . Ginkgo extensin II, carrot extensin and soybean extensins are similar in amino acid composition to the tomato extensins. Bovine serum albumin, Ginkgo extensin I, Douglas fir PHRGP-extensin, maize THRGP-extensin and Asparagus 
extensin I \& II were not crosslinked and do not have amino acid compositions similar to the tomato extensins. Comparison of motifs common to the extensins which crosslink will help to define the domain involved in crosslinking.

\section{Extensins from the non-graminaceous monocot Asparagus}

Examination of Hyp-rich glycoproteins from Asparagus, a non-graminaceous monocot, was initiated as part of a phylogenetic study of the relitionship between the non-graminaceous monocots, the graminaceous monocots and the dicots. Graminaceous walls differ significantly from dicot walls: the graminaceous wall is Hyp-poor, pectin is virtually replaced by glucuronoarabinoxylan (Jarvis \& Duncan, 1988), and the hydroxyproline-rich glycoproteins (HRGPs) are structurally distinct from dicot extensins. Recent work suggests an ancient monocot-dicot divergence (Martin et al., 1989; Wolfe et al., 1989). Non-graminaceous monocot and dicot walls are significantly similar as both are pectin-rich (Jarvis \& Duncan, 1988). Thus we asked whether the difference between the graminaceous and non-graminaceous monocot cell wall is trivial or profound.

We used cell suspension cultures and the intact cell elution technique as a general tool. Initial investigation showed a higher Hyp-content in the Asparagus cell wall than in the wall of maize. Also, from the analysis of a peptide from the wall we suspected the presence of isodityrosine (IDT). Both elevated Hyp content and presence of IDT would support our hypothesis that the non-graminaceous monocot wall is more closely related to the dicot wall than to the graminaceous monocot wall. Subsequent work led to the isolation of two salt-elutable HRGPs (I \& II) from the Asparagus wall. Characterization included neutral sugar compositions, Hyp-arab profiles, and partial sequences. Two major peptides sequenced from these HRGPs are:

Asp.I-M6: His-Lys-Pro-Hyp-Hyp-Ser-Ser-His-Leu-Pro-Hyp-Hyp-llu-Tyr

Asp.II-M4: Ser-Hyp-Hyp-Hyp-Ser-Hyp-Val-Lys-Pro-Tyr-Pro-Arg

Neutral sugars from both HRGPs and the Hyp-arab profile of one (HRGP-II) are clearly dicot-like. Also the Hyp-arab profiles of Asparagus and tomato walls are virtually identical. HF solvolysis of the Asparagus wall left most of the HRGP bound to the wall (unlike maize). We also confirmed the presence of IDT in the non-graminaceous wall, and obtained a peptide sequence closely related to the dicot P1-type extensin motif. These, along with previous data showing an intermediate Hyp-content and pectin-rich wall, support a closer relationship between non-graminaceous monocots and dicots than between graminaceous monocots and dicots.

On the other hand, the Asparagus wall and elutable HRGPs did show some graminaceous characteristics: tryptide M4 from the Asparagus HRGP-II clearly bridges the dicot P1-type and the maize THRGP P1-type sequences as follows: 
First we compare $\mathrm{M} 4$ with the major tryptide $\mathrm{H}_{20}$ from tomato type I extensin: Tomato $\mathrm{H}_{20}$ : Ser-Hyp-Hyp-Hyp-Hyp-Val-Lye-Pro-Tyr-His-Pro-Thr-Hyp-ValTyr-Lys

Asp.II-M4: Ser-Hyp-Hyp-Hyp-Ser-Hyp-Val-Lys-Pro-Thr-Pro-Arg

Now we compare M4 with the minor chymotryptide TC2 from Maize THRGP extensin:

Asp.II-M4: Ser-Hyp-Hyp-Hyp-Ser-Hyp-VaH-Lys-Pro-Thr-Pro-Arg Maize THRGP TC2: Thr-Hyp-Ser-Hyp-Lys-Pro-Thr-Hyp-Hyp-Thr-Tyr

(Note that in both instances a single insert/deletion residue is underlined, while the similarities are shown in bold face type.)

It is a common observation that some walls are Hyp-rich and some Hyp-poor. Advanced dicot walls are Hyp-rich and relatively poor in hydrophobic amino acids. Walls of other groups: monocots (graminaceous and non-graminaceous), primitive dicots, and gymnosperms tend to be Hyp-poor, but have a significant hydrophobic amino acid content. These Hyp-poor, hydrophobic walls all contain an X-Pro-Pro-Pro motif or motifs not seen in advanced dicots.

Another interesting result was a high glycine content (ca. 20 mole\%) of the Asparagus HF-solubilized wall. Glycine-rich proteins (GRPs) have recently been recognized as structural proteins in Hyp-poor walls (Condit \& Meagher, 1986; Keller et al., 1989). Keller et al. (1988) have speculated that the GRPs and HRGPs are evolutionarily related by duplication and inversion of the original gene. The codons for proline are CCX; the codons for glycine are GGX. Therefore the inversion of one gene (proline or glycine-rich) along with its transcription in the reverse orientation would result in the expression of the other protein type. Elucidation of the role of GRPs and their evolutionary relationship to the HRGPS awaits further study.

Gum arabic glycoprotein is a twisted hairy rope; a new model based on Ogalactosylhydroxyproline at the polysaccharide attachment site

Separation of the wound exudate from Acacia senegal (L.) Willd., "gum arabic," on a preparative Superose-6 column gave two major fractions: a high molecular weight gum arabic glycoprotein (GAGP) containing about $90 \%$ carbohydrate and a lower molecular weight heterogeneous gum arabic polysaccharide fraction. HFdeglycosylation of GAGP gave a large (ca. 400 residue) hydroxyproline-rich polypeptide backbone (dGAGP). Alkaline hydrolysis of GAGP showed that most of the carbohydrate was attached to the polypeptide backbone as small (ca. 30 residue) Hyp-polysaccharide substituents. After partial acid hydrolysis of the Hyppolysaccharide fraction we identified O-galactosylhydroxyproline as the glycopeptide linkage, identical with that of hydroxyproline-rich arabinogalactan proteins (AGPs). However, unlike the acidic alanine-rich AGPS, GAGP is basic 
and notably deficient in alanine. Thus, while the GAGP polypeptide backbone closely resembles a typical Hyp-rich extensin, the GAGP polysaccharide sidechains resemble AGPs. Possibly all three proteins comprise a phylogenetically related extensin superfamily of extended rodlike macromolecules. The "wattle-blossom" model for AGP and gum arabic predicts a few large polysacci:aride substituents along the polypeptide backbone of a spheroidal macromolecule. On the contrary, our data imply a rodlike molecule with numerous small polysaccharide substituents (attached to $24 \%$ of the Hyp residues), regularly arranged along a highly periodic polypeptide backbone based, hypothetically, on a 10- to 12-residue repetitive peptide motif with the empirical formula: $\mathrm{Hyp}_{4} \mathrm{Ser}_{2}$ Thr Pro Gly Leu His. Thus a simple statistical model of the gum arabic glycoprotein predicts a repeating polysaccharide-peptide subunit of about $7 \mathrm{kDa}$. The small polysaccharide substituents will maximize hydrogen bonding if aligned along the long axis of the molecule, forming in effect a twisted hairy rope. Electron micrographs of rotary shadowed GAG molecules support that prediction and may also explain how such apparently large molecules can exit the cell by endwise reptation through the small pores of the primary cell wall.

\section{Screening a maize cDNA library in lambda ZAP II to identify CDNA clones encoding HHRGPs}

Lambda ZAP II was used to transfect XL1-Blue cells. Expression of fusion proteins was induced with the lactose inducer IPTG. Plaque lifts were made using nitrocellulose filters, and these filters were incubated with polyclonal antibodies raised against the HHRGP peptide backbone.

Incubation with a second antibody conjugated with alkaline phosphatase allowed for a color reaction by positive fusion protein-producing plaques. Using this procedure, all plaques reacted. It was determined that cross-reactive antibodies from $E$. coli present in Freund's adjuvant used to produce dHHRGP antibodies make it impossible to use this method to screen for clones. Chymotrypic peptides recently sequenced from the HHRGP will allow us to have oligonucleotides with multiple degeneracy synthesized by the MSU-Department of Biochemistry Macromolecular Facility for our use in screening the cDNA library.

Affinity purification of THRGP antibodies using antigen immobilized on nitrocellulose filters. An affinity purification protocol was tested for purification of THRGP antibodies (readily on hand). THRGP was allowed to adsorb to nitrocellulose in pH 6.0 water. Adsorption as monitored at $A_{280}$ was essentially complete in 2 mins. THRGP antibodies were incubated with the filter for $2 \mathrm{hrs}$, the filters were rinsed, and bound antibody released with $\mathrm{pH} 3$ citrate buffer. Based on Bradford quantitation of protein and ELISA determination of antibody titer, affinity purification resulted in a 6.5 -fold purification relative to serum proteins. 


\section{References}

Condit, C.M., R.B. Meagher. Nature 323:178 (1986).

Janvis, M.C., W. Forsyth and H.J. Duncan. Plant Physiology 88:309 (1988).

Keller, B., N. Sauer and C.J. Lamb. EMBO J. 7:3625 (1988).

Keller, B. , M.D. Templeton and C.L. Lamb. Proc. Natl. Acad. Sci. USA 86:1529 (1989).

Martin, W., A. Gierl, and H. Saedler. Nature 339:46 (1989).

Wolfe, K.H., M. Gouy, Y.W. Yang, P.M. Sharp and W.H. Li. Proc. Natl. Acad. Sci. USA 86:6201 (1989). 


\section{INTERACTION OF NUCLEAR AND ORGANELLE GENOMES}

Lee Mclntosh. Shawn Anderson, Neil Bowlby, Linda Briggs, Susan Fujimoto, Carrie Hiser, Mary Kakefuda, Roxy Nickels, David Rhoads, Idah Sithole and Lawrence Smart

Energy conversion in plants is controlled by the expression of genes whose products make up the membrane complexes that underlie photosynthetic and respiratory electron transport. We seek to understand how the assembly of these complexes is regulated and to determine which structural components control specific electron transport events.

Photosynthetic organisms contain unique membrane complexes that perform energy transduction and that are ultimately responsible for the production of the oxygen in our atmosphere. Until recently, the study of photosynthesis at a molecular level was limited both conceptually and technically. However, investigations that combine molecular genetics and biochemistry in cyanobacteria now allow rapid elucidation of the molecular bases for photosynthesis.

Plant mitochondria differ from those of most other eukaryotes in that their genomes contain far greater amounts of DNA (Levings and Brown, 1989) which is organized in complex and little-understood patterns (Palmer and Shields, 1984). However the most important differences relative to higher plants, specifically crop plants, are functional differences (Amthor, 1984; Lambers, 1985). Possibly the most striking functional difference between plant and animal mitochondria is the presence of two terminal oxidases in higher plants (Figure 1). Plants contain a "normal" cytochrome C oxidase (Hawkesford et al., 1989) along with an "alternative" oxidase which was first identified by its resistance to cyanide (Genevois, 1929). The electrons flowing through this alternative pathway are not linked to the production of a transmembrane potential and thus are lost, or wasted, for the production of ATP. We wish to understand how respiration is controlled in higher plants and, in particular, how the distribution of electron flow between the two terminal oxidases is regulated.

\section{Molecular Bases of Photosynthesis}

\section{How is oxygen evolved?: Photosystem II}

Our immediate goal is to understand how water is "split" and oxygen evolved by photosystem II. Previously, as a first step, we demonstrated that both $D^{+}$and $Z^{+}$, the first electron acceptors from the oxidation of water, are tyrosine radicals 


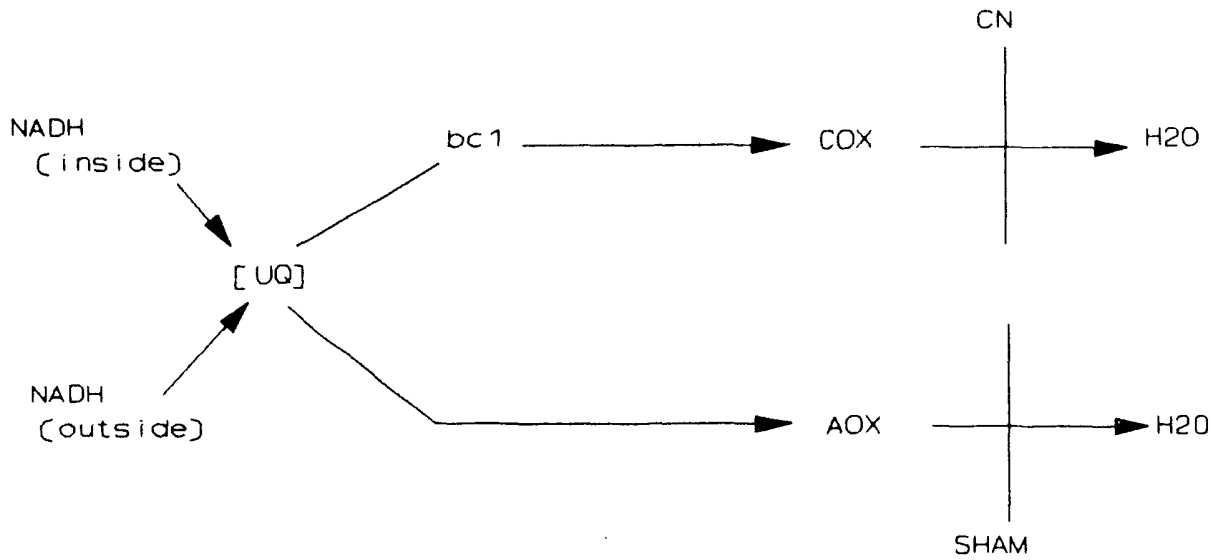

Figure 1. Schematic representation of the plant respiratory chain. $\mathrm{NADH}_{\text {(inslde) }}$ signifies the complex on the inner side of the inner membrane and $\mathrm{NADH}_{\text {(outside) }}$ the complex on the outer side of the inner membrane. Cyanide (CN) inhibits the cytochrome pathway and salicylhydroxamic acid (SHAM) is a specific inhibitor of the alternative pathway (Schonbaum et al., 1971). The cytochrome oxidase $(C O X)$ pathway and the alternative oxidase $(A O X)$ pathway branch at ubiquinone (UQ).

located on the D2 and D1 polypeptides of the photosystem II reaction center respectively (Debus et al., 1988a; 1988b). It is well known that $4 \mathrm{Mn}$ atoms are required for water oxidation (Babcock et al., 1989); however, the mechanism of water oxidation involving these Mn atoms is unknown. Therefore, we pursued two lines of investigation: first, a series of experiments which expand our investigation of $Y_{D}^{+}$and $Y_{Z}^{+}$; second, experiments aimed at the identification of the amino acid residues in the photosystem II complex which act as Mn ligands.

A series of amino acid mutations in both D1 and D2 (the reaction center "core" of photosystem II) has been made in the areas surrounding and including the redox-active tyrosines, $Y_{D}^{+}$and $Y_{Z}^{+}$. Two types of mutations have been incorporated into this strain: structural mutations and mutations made to potential $\mathrm{Mn}$ amino acid ligands. All mutants were screened initially for the ability to evolve oxygen. Four classes of mutants have been isolated: those evolving oxygen at wild-type rates, those which do not evolve oxygen, and those which evolve oxygen at approximately $33 \%$ and $66 \%$ of wild-type rates. Further analysis of these mutants has begun and includes experiments measuring the effect of light intensity on oxygen evolution and fluorescence induction kinetics. Both of these approaches have been undertaken to study the quantum efficiency of cells with altered photosystems. 
Detailed analyses of photosystem II mutants of Synechocystis 6803 require that biologically active and stable preparations of photosystem II be isolated. We have been able to obtain preparations similar to those reported in the literature (Bernap et al., 1989; Metz et al., 1989); however, these preparations still lack some properties required for all of our projected analyses. At this time our efforts to gain a purified photosystem II preparation are advancing but remain at a developmental stage.

\section{Photosystem I: Reaction center genes and structure}

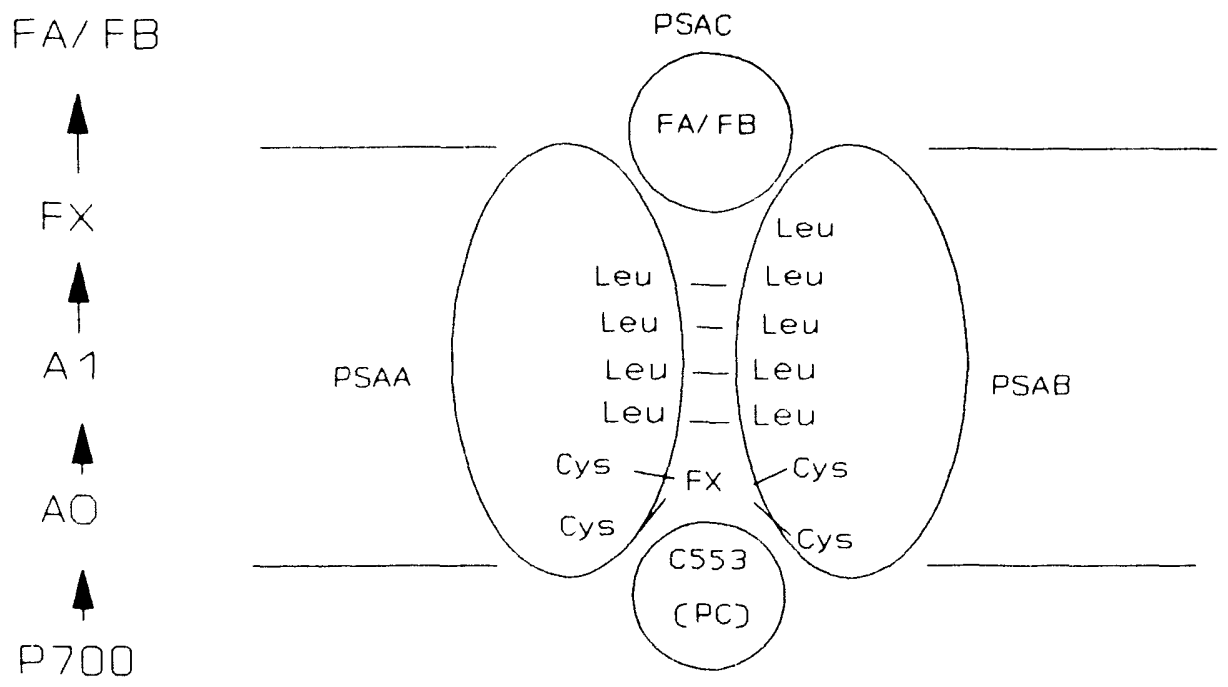

Figure 2. Photosystem I Reaction Center. The linear electron transport chain is shown on the left. On the right, a schematic representation for the reaction center core proteins (see text) with the proposed cysteine ligands for the [4Fe-4S] center $F_{X}$ are shown in relation to the positions of the leucine residues proposed as a potential "leucine zipper" in photosystem I (Webber and Malkin, 1990).

The crystal structure of the bacterial photosynthetic reaction center from Rhodopseudomonas viridis (Michel and Deisenhofer, 1988) has allowed insights into charge separation as it occurs in iron- and quinone-based systems such as the oxygenic photosystem II of higher plants and algae. It is now understood that the core polypeptides found in the bacterial reaction center have been conserved through evolution into oxygenic photosystem II (Babcock et al., 1989). Indeed, it has been found that some critical primary amino acid sequence arrangements have been preserved (Michel and Deisenhofer, 1988). In comparison to 
photosystem II, little is known concerning the detalls of electron flow through photosystem I.

The reaction center of photosystem I (Figure 2) contains the electron transfer components $\mathrm{P} 700$; a primary chlorophyll electron acceptor, $\mathrm{AO}$; a quinone intermediate electron acceptor, $\mathrm{A} 1$; and three iron-sulfur clusters, FX, FB, and FA (for review see Golbeck, 1989; Golbeck and Bryant, 1991). The core of the reaction center is usually thought to be composed of two $64 \mathrm{kDa}$ polypeptides and a $9 \mathrm{kDa}$ polypeptide, encoded by the $p s a A, p s a B$ and $p s a C$ genes, respectively. FA and $\mathrm{FB}$ are [4Fe-4S] clusters within the $9 \mathrm{kDa}$ polypeptide (Dunn and Grey, 1988) and $\mathrm{FX}$ is a [4Fe-4S] cluster possibly residing between the two $64 \mathrm{kDa}$ polypeptides (Golbeck, 1989). A major question arises concerning the mechanism of electron transfer between these three [4Fe-4S] centers. The pathway of electron flow from FX through FA and FB is unknown and knowledge concerning the assignment of the cysteine residues for these [4Fe-4S] centers is also lacking.

In order to analyze the structure and function of photosystem I we are employing "reverse genetics". That is, the reaction center genes encoding photosystem I will be employed to probe function. These experiments are dependent on the existence of a biological system in which mutants in photosystem I may be isolated. In brief, a photosynthntic organism which can be grown without a functional photosystem I is required so that mutants altered in photosystem I capacity may be segregated from wild type cells. Such a biological system must have the ability to be grown in the absence of photosystem I activity. Until recently, such a unicellular system, one whose genes are easily manipulable, has not been available. However, we have recently found that the strain of cyanobacteria most frequently employed for photosystem II mutational analyses, Synechocystis 6803, may be grown heterotrophically (Anderson and Mclntosh, 1991b). We showed that a single, five-minute pulse of light every 24 hours will allow Synechocystis 6803 to grow in complete darkness (with $5 \mathrm{mM}$ glucose). Further experiments have shown that this is a blue-light requirement; however, it is not known how this pulse of light facilitates heterotrophic growth.

The ability to grow Synechocystis 6803 heterotrophically has now allowed us to begin a molecular analysis of the reaction center of photosystem $I$ in this organism. The reaction center genes $p s a C, p s a A$ and $p s a B$ have been cloned from Synechocystis 6803. psaC, psaA and psaB have been sequenced and their expression studied in Synechocystis 6803 (Anderson and McIntosh, 1991a; Smart and Mclntosh, in preparation). In chloroplast genomes from higher plants, psaC is part of an operon: ndhE-psaC-ndhD (Shantz and Bogorad, 1988). It was found in Synechocystis 6803 that ndhE-psaC is present while only part of a sequence similar to ndhD follows psaC. Further probing of the Synechocystis genome demonstrated that a complete ndhD gene is apparently absent, thus indicating that its function may be dispensable (Anderson and Mclntosh, 1991a). 
In Synechocystis 6803, psaA and psaB are arranged in an operon as they are in higher plants (Kirsch et al., 1986) and another cyanobacterlum, Synechococcus 7002 (Cantrell and Bryant, 1987). The polypeptides encoded by psaA and psaB are $88 \%$ and $93 \%$ identical when compared with the same deduced protein sequences from Synechococcus 7002 , and are $81 \%$ and $80 \%$ identical to the same gene products from spinach (Kirsch et al., 1986). The genes are transcribed as a $5.0 \mathrm{~kb}$ transcript which is rapidly turned over in Synechocystis 6803 (Smart and Mclntosh, unpublished).

The gene products of $p s a A$ and $p s a B, P S A A$ and PSAB, have eleven proposed membrane-spanning $\alpha$-helices similar to those predicted for higher plants (Golbeck and Bryant, 1991). Four cysteines are thought to bind the [4Fe-4S] center $F_{X}$, with two of these residues residing in PSAA and two in PSAB (Golbeck and Bryant, 1991). $F_{X}$ is thus proposed to be shared by two separate polypeptides. Analysis of PSAA and PSAB sequences from a number of organisms has also shown that a series of leucine residues adjacent to the proposed iron-sulfur cysteine ligands are present as a possible "leucine zipper" shared between these two membrane proteins (Webber and Malkin, 1990). The functional significance of these leucine residues is not known, although it is possible that they may play a role in the biogenesis of the photosystem I reaction center.

As a first step in a molecular genetic analysis of photosystem I in Synechocystis 6803, we have genetically deleted the photosystem I core and recovered viable mutants grown under our conditions of blue-light-activated heterotrophic growth. The psaA gene was interrupted with a bacterial gene encoding kanamycin resistance. A mutant was selected under heterotrophic growth conditions which revealed complete segregation of a mutated psaA gene (Smart et al., in preparation). This strain apparently lacks the chlorophyll molecules associated with photosystem I and thereby appears blue in color. Photosystem II activity is present in approximately wild-type amounts and the growth rate of this mutant strain is indistinguishable from that of wild type grown under heterotrophic conditions. It is interesting to note that this strain will not grow in the presence of continuous dim light. The selection of this photosystem I mutation opens the door for further analysis of the potential redox-active amino acid residues within the photosystem reaction center, as well as those residues involved in its structural integrity and biogenesis.

\section{A copper-inducible protein in Synechocystis: Plastocyanin}

The gene encoding plastocyanin, petE, has been cloned and sequenced from Synechocystis 6803 (Briggs et al., 1990). This gene is of interest for two reasons: first, the appearance of plastocyanin is copper-dependent; second, it is a soluble redox carrier to photosystem I. A functional replacement for plastocyanin, cytochrome $\mathrm{C553}$, is used as the reductant for $\mathrm{P} 700^{+}$under normal laboratory 
growth conditions or when copper is limited (Briggs et al., 1990). petE from a filamentous cyanobacterium, Anabaena sp. PCC 7937, was previously cloned and sequenced (Van der Plas et al., 1989). It is interesting to note that the Synechocystis petE gene is more similar in protein sequence to the gene from Chlorella (Kelly and Amber, 1974) than to the sequence from Anabaena. The Synechocystis 6803 gene is present in a single copy and encodes a precursor protein of 126 amino acids. Putative promoter sites -16 and -38 base pairs from the start of the petE have been identified on the basis of sequence similarity of known bacterial sequences. Analysis of transcript pools indicate that petE in Synechocystis 6803 is not transcriptionally regulated since accumulation of its messenger RNA appears to be independent of the copper concentration in the growth media. (Supported, in part, by an REF-MSU grant: in collaboration with V. Pecoraro, Univ. Michigan.)

\section{Biochemical and Molecular Analyses of Plant Mitochondrial Respiration}

\section{Control of plant respiratory capacity: A molecular approach}

Higher plants contain two mitochondrial respiratory pathways: a "normal" cytochrome pathway which is linked to the production of a transmembrane potential, and an "alternative" pathway which is not linked to a membrane potential and thus "wastes" energy (for review, Laties, 1982). These pathways branch at ubiquinone and this common pool of ubiquinone is kept reduced via substrate oxidation by a number of dehydrogenase complexes (Figure 1). A functional role of the alternative pathway is only agreed upon for plants which display thermogenic, $\mathrm{CN}$-resistant respiration in their inflorescences. Heat produced by electron flow through the alternative pathway in these inflorescences is used to volatilize insect attractants, thus facilitating pollination (Meeuse, 1975).

A more general but hypothetical physiological role of the alternative pathway is that this pathway acts as an "overflow" and is active only when the cytochrome pathway is limited or saturated (Lambers, 1982). However, recent evidence (Dry et al., 1989) demonstrates that the overflow hypothesis cannot be correct in its entirety and thus the mechanism(s) regulating the alternative pathway remains obscure. At many points during development, ATP may be in excess, whereas carbon skeletons for metabolic processes may be limiting (Hunt and Fletcher, 1976; Moore and Rich, 1985). From this viewpoint it could be assumed that the alternative pathway would allow the TCA cycle to continue operating, thus ensuring the production of carbon skeletons for biosynthetic purposes. Although the alternative pathway has been investigated extensively at the physiological level, relatively little biochemical characterization has been reported. In order to evaluate the role of the alternative pathway for overall carbon balance in plants the complete biochemical characterization and, ultimately, genetic manipulation of the alternative oxidase is required. 
In many plant tissues wounding can induce an increased capacity for the alternative oxidase (Laties, 1982). The "classical" material for studying plant wounding and respiration has been potato tubers. We have found that upon wounding of potato tuber tissue the relative percent capacity of the alternative pathway increases while the cytochrome pathway decreases (Hiser and Mclntosh, 1990). These results basically agree with previous work (Theologis and Laties, 1978), with the exception that the increase in alternative pathway activity appears to correlate with de novo synthesis of the alternative oxidase. Partial purification of inner mitochondrial membrane protein complexes of potato, followed by probing with alternative oxidase antibodies, indicates that the potato alternative oxidase is synthesized de novo over at least the $24 \mathrm{~h}$ aging period and is composed of, at least in part, a 36-kDa membrane polypeptide (Hiser and Mclntosh, 1990).

We are also investigating the alternative-pathway respiration in Nicotiana tabacum NT1 suspension cell cultures. Preliminary results indicate that the alternative capacity of these cells is approximately $10 \%$ and may fluctuate during the growth period. Monoclonal antibodies to the Sauromatum guttatum alternative oxidase recognize the oxidase from this cell line and it appears as a single $35-\mathrm{kDa}$ polypeptide. This cell line is transformable (Mitra and An, 1989) and grows nonphotosynthetically. In cell cultures of this type the carbon balance may also be measured (Schnapp et al., 1990). These features make this a model system for future studies on the genetic alteration of plant respiratory capacity.

\section{Analyses of "non-plant" alternative oxidases}

A monoclonal antibody to the alternative oxidase of $S$. guttatum has been used to identify a set of mitochondrial polypeptides $(\mathrm{Mr}=36.5$ and $37 \mathrm{kDa})$ associated with alternative oxidase activity of Neurospora crassa mitochondria (Lambowitz et al., 1989). These results demonstrate that the alternative oxidase is highly conserved, even between plant and fungal species.

We have begun a collaboration with $\mathrm{Di}$. A. Clarkson (NYU Medical Center) to study the alternative oxidase of the African trypanosome, Trypanosoma brucei brucei (the causative agent of African sleeping sickness). Our initial experiments indicate that the T. brucei alternative oxidase (Clarkson et al., 1989) is composed of a single $36-\mathrm{kDa}$ polypeptide which reacts with a monoclonal antibody produced to the $S$. guttatum oxidase. For comparative purposes, we will clone and sequence the alternative oxidase gene(s) from this organism.

\section{Genes for the higher plant alternative oxidase}

We have recently employed the antibodies to the $S$. guttatum alternative oxidase to clone the corresponding gene from an S. guttatum cDNA library (Rhoads and Mclntosh, 1991). This gene, aox1, has been sequenced and the protein sequence 
deduced from the DNA sequence. aox1 is encoded by nuclear DNA and codes for a polypeptide with an apparent transit peptide approximately $6 \mathrm{kDa}$ in length. The mature protein is predicted to have a minimum of three alpha-helical regions. Preliminary experiments employing this cDNA as a probe show that upon induction of increased alternative oxidase capacity in the $S$. guttatum inflorescence with salicylic acid, the mRNA pool for this gene increases. Previous work (Elthon et al., 1989b) demonstrated that salicylic acid induces the appearance of the alternative oxidase proteins.

The S. guttatum cDNA has been employed as a probe to obtain cDNA clones from potato and rice. A partial clone from rice has been obtained (Nickels and Mclntosh, unpublished) and a full-length clone from potato has been isolated and sequenced (Hiser and Mclntosh, unpublished). Comparison of the deduced amino acid sequence with that of the $S$. guttatum sequence shows that the most highly conserved areas are those predicted to form $\alpha$-helical regions (at least two of which are predicted to be membrane helices). The potato sequence starts with a transit sequence that is very dissimilar to that from $S$. guttatum aox1. Southern blotting with potato genomic DNA indicates that the potato aox1 gene is present is low copy number.

\section{References}

Amthor, J.S. Plant Cell and Environ. 7:561 (1984).

Anderson, S.L. and L. Mclntosh. Plant. Mol. Biol. in press (1991a).

Anderson, S.L. and L. Mclntosh. J. Bacteriol. in press (1991b).

Babcock, G.T., B.A. Barry, R.J. Debus, C.W. Hoganson, M. Atamian, L. McIntosh, I. Sithole and C.F. Yocum. Biochemistry 28:9557 (1989).

Burknap, R.L., H. Koike, G. Sotiropoulou and L.A. Sherman. Photosyn. Res. 22:123 (1989).

Briggs, L., V.L. Pecoraro and L. Mclntosh. Plant Mol. Biol. 15:633 (1990).

Cantrell, A. and D.A. Bryant. Plant Mol. Biol. 9:453 (1987).

Clarkson, A.B., E.J. Bienen, G. Pollakis and R.W. Grady. J. Biol. Chem. 264:17760 (1989).

Debus, R.J., B.A. Barry, G.T. Babcock and L. Mclntosh. Proc. Natl. Acad. Sci. USA 85:427 (1988a).

Debus, R.J., B.A. Barry, I. Sithole, G.T. Babcock and L. Mclntosh. Biochemistry 27:9071 (1988b).

Dry, I.B., A.L. Moore, D.A. Day and J.T. Wiskich. Arch. Biochem. Biophys. 273:148 (1989).

Dunn, P.P.J. and J.C. Gray. Plant Molec. Biol. 11:311 (1988).

Elthon, T.E., R.L. Nickels and L. Mclntosh. Plant Physiol. 89:1311 (1989a).

Elthon, T.E., R.L. Nickels and L. Mclntosh. Planta 180:82 (1989b).

Genevois, M.L. Rev. Gen. Bot. 41:252 (1929).

Golbeck, J.H. Biochim. Biophys. Acta 895:167 (1989).

Golbeck, J.H. and D.A. Bryant. Curr. Topics Bioenerg. In press (1991). 
Hawkesford, M.J., A.D. Liddell and C.J. Leaver. Plant Physiol. 91:1535 (1989).

Hiser, C. and L. Mclntosh. Plant Physiol. 93:312 (1990).

Hunt, L. and J.S. Fletcher. Plant. Physiol. 57:304 (1976).

Kelly, J. and R.P. Amber. Biochem. J. 143:681 (1974).

Kirsch, W., P. Seyer and R.G. Hermann. Curr. Genet. 10:843 (1986).

Lambers, H. Plant Physiol. 55:478 (1982).

Lambers, H. In: Higher Plant Cell Respiration (R. Douce and D.A. Day, eds.), Springer, Berlin, pp. 418-473 (1985).

Lambowitz, A.M., J.R. Sabourin, H. Bertrand, R. Nickels and L. Mclntosh. Molec. Cell. Biochem. 9:1362 (1989).

Latles, G.G. Ann. Rev. Plant Physiol. 33:519 (1982).

Levings, C.S. and G.G. Brown. Cell 56:171 (1989).

Meeuse, B.J.D. Ann. Rev. Plant Physiol. 26:117 (1975).

Metz, J.G., P.J. Nixon, M. Rogner, G.W. Brudvig and B.A. Diner. Biochemistry 28:6960 (1989).

Michel, H., and J. Diesenhofer. Biochemistry 27:1 (1988).

Mitra, A. and G. An. Mol. Gen. Genet. 215:294 (1989).

Moore, A.L. and P.R. Rich. In: Higher Plant Cell Respiration (R. Douce and D.A. Day, eds.) Springer, Berlin, pp. 134-172 (1985).

Palmer, J.D. and C.R., Shields. Nature 307:437 (1984).

Rhoads, D.R. and L. Mclntosh. Proc. Natl. Acad. Sci. USA in press (1991).

Schnapp, S.R., R.A. Bressan and P.M. Hasegawa. Plant Physiol. 93:384 (1990).

Schonbaum, G.R., W.D. Bonner, B.T. Story and J.T. Bahr. Plant Physiol. 47:124 (1971).

Shantz, R. and L. Bogorad. Plart Molec. Biol. 11:239 (1988).

Theologis, A. and G.G. Laties. Plant Physiol. 62:232 (1978).

Thomas, W.E. and D.J. Ellar. J. Cell Sci. 60:181-197 (1983).

Van der Plas, J., A. Bovy, F. Kruyt, G. de Vrieze, E. Dassen, B. Klein and P. Weisbeek. Molec. Microbiol. 3:275 (1989).

Webber, A.N. and R. Malkin. FEBS :ett. 264:1 (1990). 


\section{SENSORY TRANSDUCTION IN PLANTS}

Kenneth Poff. Thérèse Best, Bertha Bullen, Marie-Claude Fortin, Abdul-Kader Janoudi, Radimir Konjevic, Vladimir Orbovic and Elizabeth Rosen

Plants acquire information concerning their environment by processing a number of environmental stimuli such as light, gravity, water, touch, $\mathrm{CO}_{2}$ and temperature. Until recently, the responses of plants to these stimuli have been known almost totally at the phenomenological level. Ultimately, the sensory physiologist wishes to understand the reception and processing of sensory information at both the behavioral/physiological and molecular levels. In this laboratory, we have been studying tropistic responses to three different stimuli. Specifically, we have been investigating hypocotyl and root gravitropism, root thermotropism and phototropism. We are moving the level of understanding of these responses more to the nechanistic level largely through physiological and biophysical characterization of mutants that have alterations in specific components of the transduction sequences.

For each sensory pathway, one would like answers to the following three questions. What is the identity of the receptor molecule(s)? What is the mechanism for obtaining directional information? What are the steps in the transduction sequence? Root thermotropism is very poorly understood. We have now provided convincing evidence that thermotropism exists, and have also described the dependence of thermotropism on the strength of the stimulus, and on the pre-treatment and exposure temperatures. However, we do not know the ariswer to any of the above three questions.

Knowledge of gravitropism is only slightly greater. Here also, answers to the three questions above are not known. However, the descriptive physiology is now well known, and we have recently described a set of mutants with altered capacity to respond to gravity (B'sllen et al., 1990).

Phototropism is better understood than thermotropism and gravitropism. Although the photoreceptor pigment has not been isolated ar.t identified, it is thought to be a flavoprotein (Poff and Butler, 1974; Pohl and Pusso, 1984; Vierstra and Poff, 1981). It is now known that the plant obtains directional information by measuring light on its lighted and shaded sides, with the shading being provided by scatter and absorption primarily by carotenoids (Poff, 1983; Piening and Poff, 1988). One major impediment to the understanding of phototropism has been the complexity of the stimulus-response relationship. We are now recognizing that this complexity is derived from a process of adaptation which proceeds along with the phototropic response itself and which alters the capacity of the organism to respond to the light (Janoudl and Poff, 1991). As reported below, adaptation involves not only the expected blue light photoreceptor pigment, but also phytochrome. Unfortunately, not one 
intermediate in the sensory transduction pathway has been identified. We are approaching this problem by selecting and characterizing mutants with alterations in specific steps of the transduction sequence. We are characterizing the mutants physiologically, biophysically, and genetically to identify those mutants which are altered in some critical step in phototropism. It is our hope that we will be able to use these mutants to clone the genes of interest and thereby determine the proteins associated with particular steps in the transduction sequence. (Supported, in part, under grant \# NAGW-882 from NASA, a NASA pre-doctoral fellowship to B. Bullen. M.-C. Fortin was supported by Agriculture Research Canada.)

\section{Enhancement of phototropism in Arabidopsis thaliana is controlled by phytochrome}

We have shown that the capacity of etiolated Arabidopsis thaliana seedlings to respond phototropically is altered by a previous exposure to light (Janoudi and Poff, 1991). This alteration, which we refer to as adaptation, consists of several components. Within seconds of an exposure to a saturating pulse of blue light, the seedlings are desensitized. That is, the seedlings show no response to a pulse of unilateral blue light which would have induced normal phototropism in the absence of the blue pre-irradiation. The capacity of the seedlings to respond phototropically recovers in about 10-20 min. Following recovery, the phototropic responsiveness is slowly enhanced. In contrast with desensitization which is induced only by blue light, enhancement can be induced by both blue and red pre-irradiation. Thus, a pre-irradiation with red light $(669 \mathrm{~nm})$ results in a 200-500 $\%$ enhancement of the phototropic response induced by subsequent unilateral blue light.

Fluence response relationships for the enhancement of phototropic curvature have been measured for several wavelengths of light. An action spectrum for enhancement (Fig. 1), derived from these fluence response relationships, is virtually identical to the absorption spectrum of the red absorbing form of phytochrome. Preliminary results indicate that the red light-induced enhancement is partially reversible by a far red light exposure given immediately after the red light pulse. We attribute this lack of complete photoreversibility to a rapid escape time for phytochrome action. The long hypocotyl and phytochrome deficient mutani of $A$. thaliana, hy2 (Koornneef, et al., 1980), also exhibited red light enhancement of phototropic curvature. Although enhancement of the mutant is similar to that of the wild-type, a higher fluence of 669-nm light may be required by hy2. Consequently, the amount of phytochrome required for the mediation of enhancement is quite small ai id is probably spectrophotometrically undetectable. 


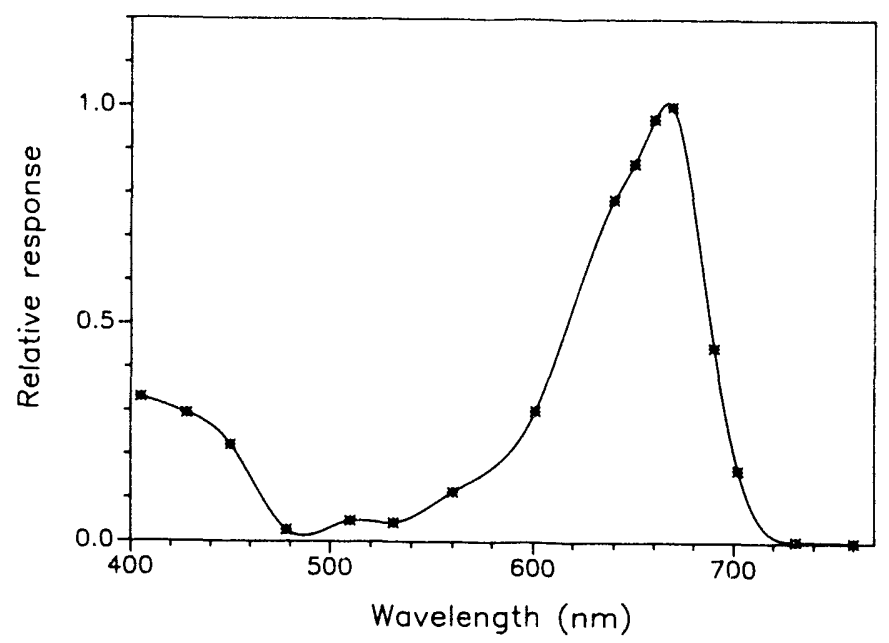

Figure 1. Action spectrum for the enhancement of phototropism by etiolated seedlings of Arabidopsis thaliana. Seedlings were pre-irradiated with light of the indicated wavelength at a minimum of 12 different fluences. Two hours later, the seedlings were irradiated with a phototropically inductive, unilateral pulse of blue

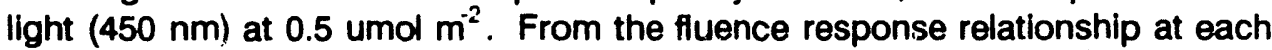
wavelength, the fluence required for the induction of $25^{\circ}$ curvature ( $14^{\circ}$ above that which would have been observed in the absence of the pre-irradiation) was determined, and the reciprocal of that fluence in umol $\mathrm{m}^{-2}$ is plotted as relative response on the abscissa as a function of the pre-irradiation wavelength.

\section{The time threshold required for second positive phototropism is altered by a red light pre-irradiation}

Janoudi and Poff (1990) showed that first positive phototropism and second positive phototropism exhibit the same fluence threshold. Second positive phototropism also exhibits a time threshold in addition to its fluence threshold. If the time threshold is measured for etiolated A. thaliana seedlings which had not been preirradiated, curvature is seen at times of unilateral blue irradiation in excess of about 10-12 min (Fig. 2). However, if seedlings are first pre-irradiated with red

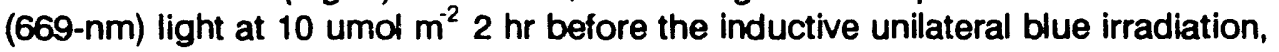
curvature is seen at times of unilateral blue irradiation exceeding about $4 \mathrm{~min}$ (Fig. 2). This decrease in the time threshold is proportional to the fluence of the red light pre-irradiation. A similar red light-induced decrease in the time threshold for second positive curvature was observed in seedings of the phytochrome-deficient mutant, hy2. We believe that this dependence of the time threshold for second positive curvature on the pre-irradiation of the seedlings with red light may account for the variable second positive time thresholds reported in the phototropism literature. These data provide the first direct evidence that phytochrome, through its absorption of blue light, modulates the subsequent phototropic response to blue light absorbed by the blue light photoreceptor pigment. 


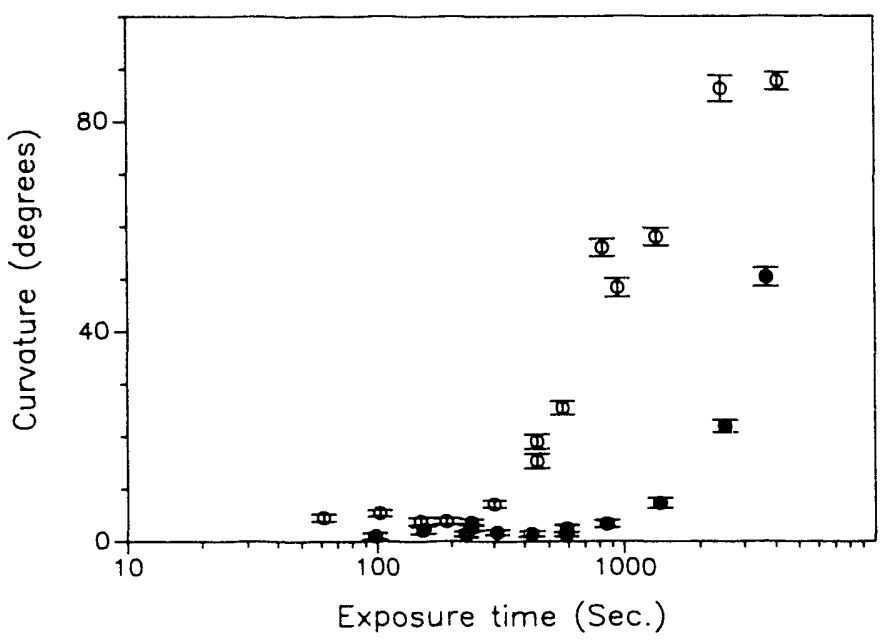

Figure 2. Dependence of the time threshold for second positive phototropism on pre-irradiation with red light. Etiolated seedlings of $A$. thaliana were either pre-

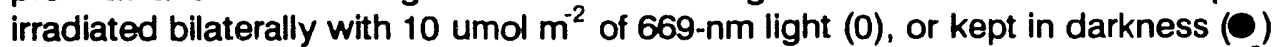

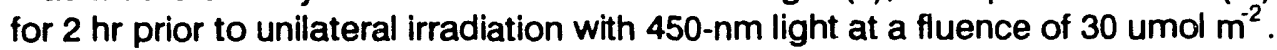
Unilateral irradiation was for the times indicated on the $x$-axis, and curvature was measured $70 \mathrm{~min}$ after the end of the irradiation.

The role of carotenoids in first positive phototropism by etiolated seedlings of Arabidopsis thaliana

A mutant of Arabidopsis thaliana, chosen for its pale cotyledon phenotype in etiolated material, has been obtained from Klaus Apel (ETH-Zentrum, Zurich, Switzerland). Fluence response curves for this putative carotenoidless mutant and its wild-type parent have been measured. The shape of the fluence response curve for the pale mutant is similar to that of its wild-type parent. However, the amplitude of curvature by the mutant is considerably lower than that of the wildtype. If the amplitude of the curvature is enhanced with a red light pre-irradiation, peaks of the two photoreceptor pigments, $P_{1}$ and $P_{11}$ (Konjevic et al., 1989) can be seen in both the pale mutant and its wild-type parent. Based on these data, we conclude that the carotenoid functions as a screen, increasing the difference in absorption on the front and rear of the seedling. We further conclude that the carotenoid is neither photoreceptor pigment.

Kinetics for first positive phototropism by etiolated seedlings of Arabidopsis thaliana

An infra-red (IR) sensitive imaging system was used to monitor the time course of first positive phototropism under radiation to which the organism does not respond, "physiological darkness". Phototropism was induced with blue light (BL) 
at $0.34 \mathrm{umol} \mathrm{m}^{-2}$, and the etiolated seedlings were placed elther on a stationary stage or mounted on a clinostat.

Each time course shows a lag time following the BL pulse. During this lag phase, the seedling shows no measurable curvature. Following the lag period, curvature toward the light source increases with time to a maximum and then decreases in a straightening phase. An average time course for phototropic curvature by a number of stationary seedlings shows a lag phase of about 10-20 min, and an average maximum curvature of $16.4^{\circ} 80 \mathrm{~min}$ after photostimulation (Fig. 3). Following maximum curvature, straightening accounted for a loss of $6.4^{\circ}$ of curvature by $180 \mathrm{~min}$ after the BL pulse. Control seedlings, treated in the same manner as the experimental seedlings but not irradiated with $B L$, showed no significant curvature (Fig. 3,4).

To investigate the role of gravity in the straightening response, seedlings were rotated on a clinostat immediately following photostimulation. The time course for phototropic curvature of such seedlings (Fig. 4) is similar to the time course for the stationary seedlings. The lag phase of $15 \mathrm{~min}$, average maximum curvature of $17.7^{\circ}$ and the average final curvature of $10.1^{\circ}$ for the seedlings on the clinostat is essentially the same as that for stationary seedlings. However, the average length of time to reach maximum curvature was slightly greater (90 $\mathrm{min}$ ) for the seedlings on the clinostat than for the stationary seedlings. In addition, the straightening phase was initiated more rapidly by the stationary seedlings than by those on the clinostat.

The effect of red light on the curvature and straightening was examined by measuring the time course for phototropic curvature of seedlings which had been pre-irradiated with $60 \mathrm{~min}$ of red light beginning $120 \mathrm{~min}$ before the BL pulse. The results (Fig. 5) show a curve shape which does not appear to be significantly different from that for seedlings not pre-irradiated with red light (Fig. 3). The lag phase, time to maximum curvature, and straightening are similar. However, the amplitude of the curvature is greater for the seedlings pre-irradiated with red light than for the non-pre-irradiated controls.

On the basis of these results, we conclude that gravity is not the primary cause for the straightening, although there is a small effect of gravity on the straightening. A red light pre-irradiation affects only the amplitude of the subsequent curvature to blue light and does not affect either the kinetics for the development of curvature or straightening. 

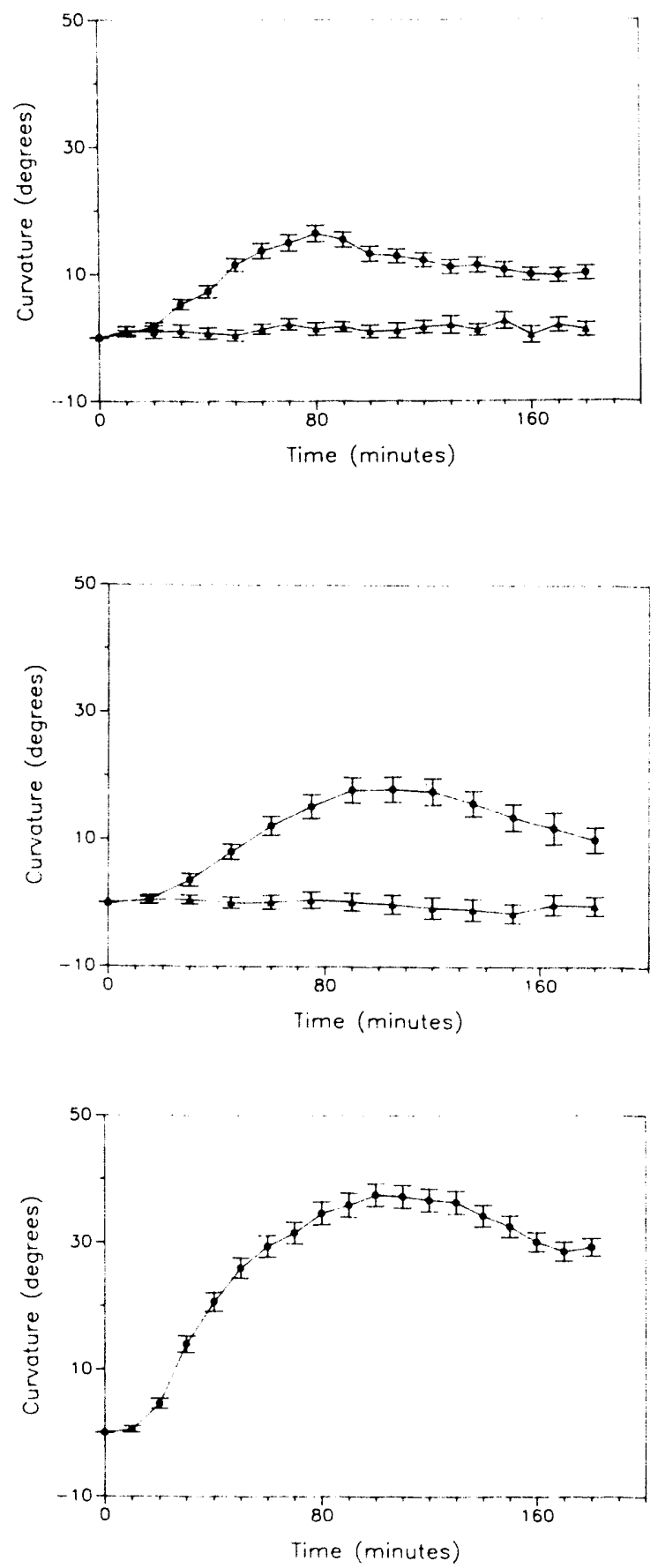

Figure 3. Average kinetics for phototropic curvature by stationary seedlings. Lower curve, unirradiated controls; upper curve, irradiated with unilateral 450-nm light. Each data point represents the average of the curvatures of at least 30 seedlings. Vertical bars represent \pm $1 \mathrm{SE}$.

Figure 4. Average kinetics for phototropic curvature by seedlings permitted to curve on a clinostat. Lower curve, unirradiated controls; upper curve, irradiated with unilateral $450-\mathrm{nm}$ light. Earh data point represents the average of the curvatures of at least 30 seedlings. Vertical bars represent $\pm 1 \mathrm{SE}$.

Figure 5. Average kinetics for phototropic curvature by stationary seedlings preirradiated with red light, and subsequently irradiated with unilateral $450-\mathrm{nm}$ light. Each data point represents the average of the curvatures of at least 30 seedlings. Vertical bars represent \pm 1 SE. 


\section{Physiological and genetic characterization of gravitropism mutants in Arabidopsis thaliana}

Four gravitropism mutant phenotypes in Arabidopsis thaliana have been identified (Bullen et al., 1990). One of these, the "random" or agravitropic phenotype, is of particular interest since it contains the best candidates for perception, or early transduction, mutants.

Studies with four mutant strains are summarized in Table I. All four mutants appear to carry single recessive nuclear mutations with respect to both the gravitropism and starch phenotypes. Although the three complementation groups for gravitropism and starch correspond, there is no correlation between the starch and gravitropism phenotypes. For example, MG-32 and MG-65 are agravitropic lines which contain normal levels of starch. However, the agravitropic mutant MG-212 contains only 5\% of wild-type starch levels. Therefore, the role of starch in gravitropism remains ill-defined.

TABLE I: Characteristics of Selected Gravitropism Mutants

\begin{tabular}{|c|c|c|c|c|c|}
\hline Mutant & $\begin{array}{l}\text { Gravi } \\
\text { Root }\end{array}$ & $\begin{array}{l}\text { Spism' } \\
\text { Shoot }\end{array}$ & Starch ${ }^{2}$ & $\begin{array}{c}\text { Complementation } \\
\text { Group }\end{array}$ & $\begin{array}{l}\text { Approx. } \\
\text { Threshold }\end{array}$ \\
\hline MG-20 & $W T$ & Alt & $10 \% W T$ & 2 & $2.7 \mathrm{~h}$ \\
\hline MG-32 & Ran & Ran & $100 \%$ WT & 3 & $5.6 \mathrm{~h}$ \\
\hline$M G-65$ & Ran & Ran & $100 \% \mathrm{WT}$ & 3 & $5.6 \mathrm{~h}$ \\
\hline MG-212 & 2 Ran & Ran & $5 \% W T$ & 1 & $2.7 \mathrm{~h}$ \\
\hline
\end{tabular}

Dose-response curves for the wild-type parent and each of the four mutants have been measured in etiolated seedlings. The wild-type parent exhibits two thresholds. The first occurs between 10 and 30 seconds of stimulation at $1 \mathrm{~g}$. After a peak response of $10^{\circ}$ curvature at 5.5 minutes of stimulation, curvature decreases to nearly zero until doses exceed the second threshold of 1.6 to 2.7 hours at $1 \mathrm{~g}$. Doses above 2.7 hours in wild-type lead to steadily increasing curvatures. All four mutants examined appear to lack the first response entirely. Three of the mutants also exhibit a slight shift to higher doses in the second threshold (Table I). Such threshold shifts may indicate an alteration in gravity perception.

In all five mutants, the gravitropism phenotype appears to be repaired when the seedlings are not etiolated. That is, seedlings grown in white light prior to 
gravistimulation in darkness respond in a wild-type manner. This relationship between light exposure and the response of the plant to gravity may help explain the wide variability present in the literature with regard to gravitropism thresholds.

\section{Thermotropism in primary roots of maize}

We have previously described thermotropism in primary roots of Zea mays L. (Fortin and Poff, 1990). We have now further characterized thermotropism as a function of gradient strength, the temperature of exposure, the pre-treatment temperature, and gravitropic stimulation. The magnitude of the thermotropic response decreases with increasing gradient strength. The temperature for maximum thermotropism is independent of gradient strength and pre-treatment temperature. However, the range of temperatures for positive and negative thermotropism varies with gradient strength. In general, temperatures lower than $25^{\circ} \mathrm{C}$ result in positive tropic responses while temperatures of $39^{\circ} \mathrm{C}$ or more result in negative tropic responses. In addition, thermotropism can modify or even reverse the normal gravitropic curvature of a horizontal root when an appropriate thermal gradient is applied opposite to the $1 \mathrm{~g}$ vector. We conclude that root thermotropism is a consequence of thermal sensing and that the curvature of the primary root results from the interaction of the thermal and gravitational sensing systems.

\section{References}

Bullen, B.L., T.R. Best, M.M. Gregg, S.E. Barsel, and K.L. Poff. Plant Physiol. 93: 525 (1990).

Bunsen, R. and H. E. Roscoe. Philos Trans. R. Soc. Lond. 153:139 (1863).

Fortin, M.-C. and K. Poff. Plant Physiol. 94:367 (1990).

Haig, C. Proc. Natl. Acad. Sci. USA 20:476 (1934).

Janoudi, A. and K. Poff. Plant Physiol. 94:1605 (1990).

Janoudi, A. and K. Poff. Plant Physiol. 95:517 (1991).

Konjevic, R., B. Steinitz and K. Poff. Proc. Natl. Acad. Sci. USA 86:9876 (1989).

Koornneef, M., E. Rolff and C. J. P. Spruit. Z. Pflanzenphysiol. 100:147 (1980).

Lin, T.P., T. Caspar, C. Somerville, and J. Preiss. Plant Physiol. 86:1131 (1988).

Loeb, J. and J. H. Northrop. Proc. Natl. Acad. Sci. USA 3:539 (1917).

Piening, C. and K. Poff. Plant Cell Environ. 11:143 (1988).

Poff, K. Phil. Trans. R. Soc. Lond. B 303:479 (1983).

Poff, K. and W. Butler. Nature 248:799 (1974).

Pohl, U. and V. Russo. In: Membranes and Sensory Transduction (Colombetti, G. and F. Lenci, eds.), pp. 231-330, Plenum Press, New York (1984).

Vierstra, R. and K. Poff. Plant Physiol. 67:1011-1015 (1981).

Wilkins, M. B. Ann. Rev. Plant Physiol. 17:379 (1966). 


\section{MOLECULAR MECHANISMS OF TRAFFICKING IN THE PLANT CELL}

Natasha Raikhel. Sebastian Y. Bednarek, Willem Broekaert, James E. Dombrowski, Hyung-II Lee, David R. Lerner, Martin Schroeder, Mark Shieh, Marguerite J. Varagona and Thea A. Wilkins

Maintenance of separate subcellular compartments in eukaryotic cells depends on the correct sorting and targeting of newly synthesized proteins. Thus, mechanisms must exist in the cell to assure that these proteins are targeted to, and subsequently translocated across, the correct intracellular membranes (Fig.1). We are working with proteins destined for different compartments: vacuoles, cell wall and nucleus. There is increasing evidence that proteins destined for subcellular organelles contain a "sorting signal" which dictates the compartment to which the protein is targeted. We are interested in understanding the molecular determinants of differential protein compartmentalization and in identifying the components of the molecular machinery which carry out the sorting process. Our goals include the analysis and characterization of protein sequences responsible for sorting to the vacuole and to the nucleus and the identification and isolation of putative receptors which recognize these sorting sequences and subsequently mediate protein transport to vacuoles and to nucleus. The nature of the sorting signal and receptor structure will provide essential background for our understanding of targeting mechanisms in cells and allow us to redirect proteins of interest either to the vacuoles or to the nucleus.

\section{SCHEMATIC REPRESENTATION \\ OF INTRACELLULAR \\ SORTING IN PLANT CELLS}

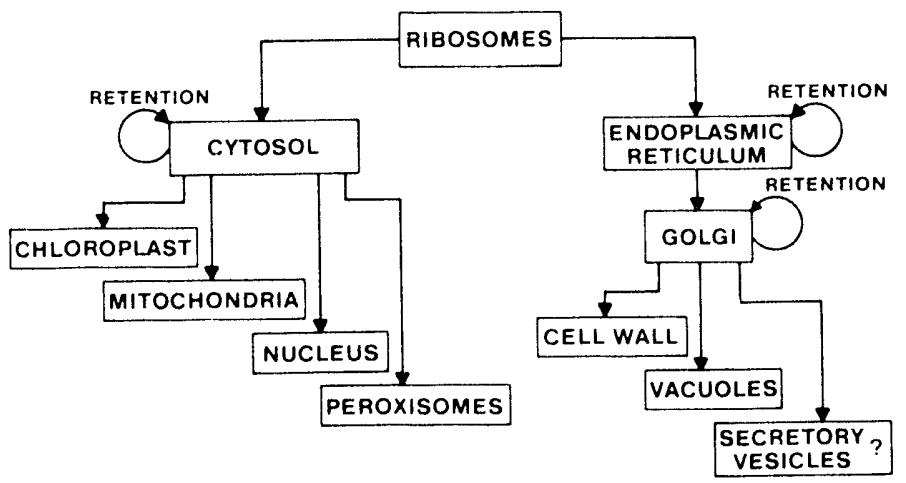

Fig.1. Schematic representation of intracellular sorting in plants cells.

Plants possess a large arsenal of both constitutive and inducible biochemical and mechanical defense mechanisms against various potential fungal pathogens and insect pests. Several arguments support the hypothesis that chitin-binding proteins are involved in host-defense: 1) these lectins exhibit high binding affinity 
for poly-N-acetylglucosamine (chitin), which is a major component of the fungal cell wall and the peritrophic membrane of the insect midgut; 2) in in vitro experiments chitin-binding proteins show insecticidal and antifungal activities; 3 ) chitin-binding proteins are predominantly located in the protective peripheral tissues of plant organs and almost all of them are wound-inducible (Chrispeels and Raikhel, 1991). We hope that by studying the molecular mechanisms of regulation and expression of genes for chitin-binding proteins we shall come to understand the endogenous function of the proteins that they encode.

\section{Role of propeptide glycan and a carboxyl-terminal propeptide in post- transiational processing and sorting of barley lectin to vacuoles in transgenic tobacco}

Mature barley lectin is a dimeric protein comprised of two identical $18 \mathrm{kD}$ polypeptides. The subunits of barley lectin are initially synthesized as glycosylated proproteins which are post-translationally piocessed to the mature protein preceding or commensurate with deposition of barley lectin in vacuoles (Lerner and Raikhel, 1989). To investigate the functional role of the glycan in the processing and intracellular transport of barley lectin to vacuoles, the sole $\mathrm{N}$ linked glycosylation site residing within the $\mathrm{COOH}$-terminal propeptide of barley lectin was altered by site-directed mutagenesis. cDNA clones encoding wild-type (wt) or glycosylation-minus $(g / y)$ barley lectin preproproteins were placed under the transcriptional control of the cauliflower mosaic virus $35 \mathrm{~S}$ promoter and introduced into Nicotiana tabacum cv. Wisconsin 38. Barley lectin synthesized from both the wt or gly constructs was processed and correctly targeted to vacuoles of tobacco leaves. Localization of barley lectin in vacuoles processed from the nonglycosylated gly proprotein indicated that the high mannose glycan of the barley lectin proprotein was not essential for targeting barley lectin to vacuoles. However, pulse-chase labeling experiments demonstrated that the glycosylated $w t$ proprotein and the nonglycosylated gly proprotein were differentially processed to the mature protein and transported from the Golgi complex at different rates. These results implicate an indirect functional role for the glycan in post-translational processing and transport of barley lectin to vacuoles (Wilkins et al., 1990). Barley lectin is synthesized as a preproprotein with a glycosylated carboxyl-terminal propeptide (CTPP) which is removed prior to or concomitant with deposition of the mature protein in vacuoles. To investigate the functional role of the CTPP in processing, assembly and sorting of barley lectin to vacuoles, a mutant barley lectin CDNA clone lacking the 15 amino acid CTPP was prepared. The CTPP deletion mutant of barley lectin was expressed in tobacco protoplasts, suspension-cultured cells and transgenic plants. In all three systems the wild-type barley lectin was sorted to vacuoles, whereas the mutant barley lectin was secreted to the incubation media (Fig.2). Therefore, we conclude that the carboxyl-terminal domain of the barley lectin proprotein is necessary for the efficient sorting of this protein to plant cell vacuoles (Bednarek et al., 1990). (Supported in part, by NSF grant DCB-9002652.) 


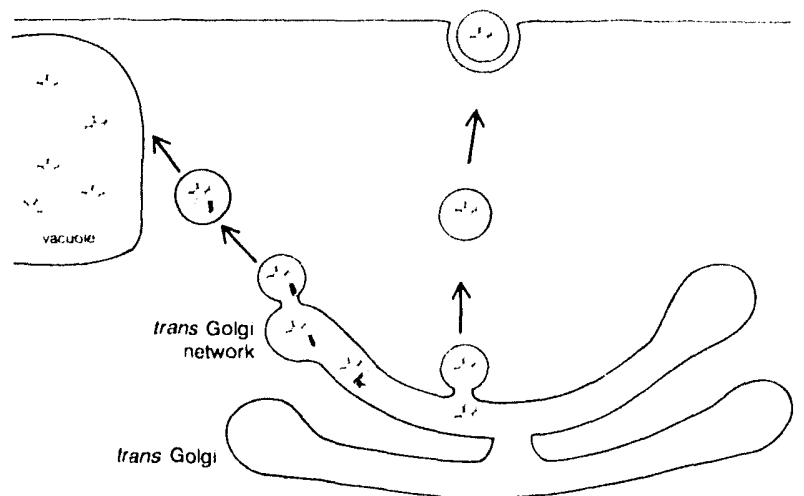

Fig.2. Model for barley lectin sorting in the trans-golgi network. The schematic representation of one subunit of a barley lectin dimer was adapted from the crystal structure of WGA (Wright, 1987). The CTPP of barley lectin is necessary for sorting of this protein to vacuoles. The barley lectin mutant lacking this sorting signal is secreted.

\section{Wound-induced accumulation of mRNA containing a hevein sequence in laticifers of rubber tree (Hevea brasiliensis) and co- and post-translational processing of the hevein preproprotein}

Hevein is a chitin-binding protein which is present in laticifers of the rubber tree (Hevea brasiliensis). A cDNA clone (HEV1) encoding hevein was isolated by polymerase chain reaction using mixed oligonucleotides corresponding to two regions of hevein as primers and a Hevea latex cDNA library as a template (Broekaert et al., 1990). HEV1 is 1018 bp long and includes an open reading frame of 204 amino acids. The deduced amino acid sequence contains a putative signal sequence of 17 amino acid residues followed by a 187 amino acid polypeptide. This polypeptide has two striking features. The amino-terminal region (43 amino acids) is identical to hevein and shows homology to several chitin-binding proteins and to the amino-termini of wound-inducible proteins in potato and poplar. The carboxyl-terminal portion of the polypeptide (144 amino acids) is $74-79 \%$ homologous to the carboxyl-terminal region of wound-inducible genes of potato. To investigate the mechanisms involved in processing of the protein encoded by HEV1, three domain-specific antisera were raised against fusion proteins harboring the amino-terminal domain, carboxyl-terminal domain and both domains. Translocation experiments using an in vitro translation system showed that the first 17 amino acid sequence functions as a signal peptide. In immunoblotting analysis of in vivo proteins from the lutoid body-enriched fraction, a 5-kD protein comigrates with purified mature hevein and crossreacts with antibodies to the amino-terminus and to the two domains (Fig.3). A 14-kD protein is recognized by antibodies to the carboxy-terminus and to the two domains. A $20-k D$ protein is cross-reactive with all three antibodies. It appears that the 5-kD (amino-terminal domain) and 14-kD (carboxyl-terminal domain) proteins are posttranslational cle avage products of the 20-kD protein (both domains) which 
corresponds to the proprotein encoded by HEV1. In addition, it is found that the amino-terminal domain can provide chitin-binding properties to the protein bearing it elther amino-terminally or carboxyl-terminally. Wounding, as well as application of the plant hormones abscisic acld and ethylene, resulted in accumulation of hevein transcripts in leaves, stems and latex, but not in roots.

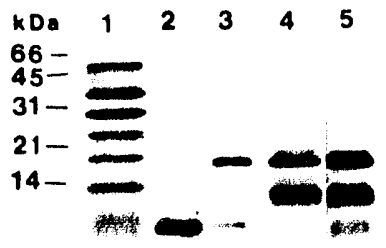

Fig.3. Immunoblot analysis of proteins in a lutoid body-enriched fraction using domain-specific antibodies. Approximately $10 \mu \mathrm{g}$ of total proteins from a lutoid body-enriched fraction was loaded on a $17.5 \%$ SDS gel. After electrophoresis, proteins were either stained with Commassie Blue (lane 1) or immunoblotted with antibodies specific for the N (lane 3), C (lane 4) or N-C (lane 5) domain. As a control, HPLC-purified hevein was immunoblotted with $\mathrm{N}$ domain-specific antibody (lane 2).

\section{Monocot regulatory protein Qpaque 2 is localized in the nucleus of maize endosperm and of transformed tobacco plants}

Protein targeting to the nucleus has been studied extensively in animal and yeast systems; however, nothing is known about nuclear targeting in plants. The opaque $2\left(\mathrm{O}_{2}\right)$ gene produces a regulatory protein that is responsible for inducing transcription of the $\alpha$-zein class of storage proteins in maize kernels. The cloned Q2 gene encodes a protein that contains a leucine zipper DNA binding domain that can interact with zein gene promoters. We have used immunolocalization to show that the $\mathrm{O} 2$ protein is present in nuclei in the maize endosperm tissues known to produce $\alpha$-zeins. In addition, neither embryonic tissue from wild-type kernels nor endosperm from kernels harboring a null 02 allele contain the $\mathrm{O} 2$ protein. Analysis of a transposable element-induced 02 allele, $02-\mathrm{m} 20$, revealed that sectors of endosperm cells contained the nuclear-localized $\mathrm{O} 2$ protein, indicating excision of the transposable element. To further study the transport of the $\mathrm{O} 2$ protein into the nucleus, we have transformed this gene, under the control of a constitutive promoter, into tobacco. Plants were shown to have detectable steady state of levels $\mathrm{O} 2$ mRNA and $\mathrm{O} 2$ protein (Fig.4). Immunolocalization of 02 protein in transformed tobacco plants indicated that the $\mathrm{O} 2$ protein was transported into tobacco nuclei. Therefore, we have developed a system to study 
nuclear targeting in plants and have established that the nuclear transport machinery is similar in monocots and dicots (Varagona et al., 1991).

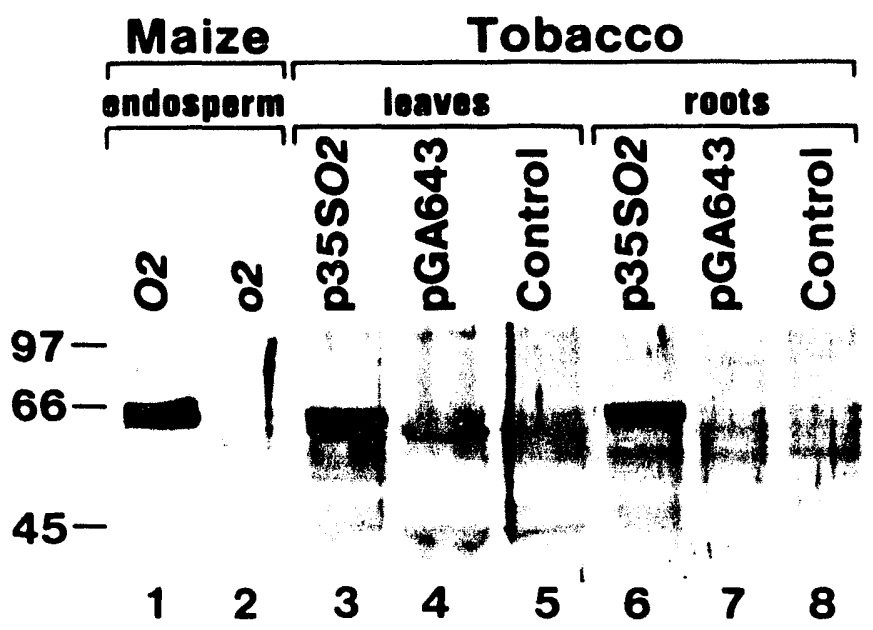

Fig.4. Analysis of steady-state levels of $\mathrm{O} 2$ protein in maize and tobacco plants. The protein gel blot contains $5 \mu \mathrm{g}$ of partially purified proteins from maize endosperm tissue harboring the $\mathrm{O} 2$ allele (lane 1), maize endosperm tissue harboring the null 02 allele (lane 2), and crude extracts from $15 \mu \mathrm{g}$ of tobacco leaves and roots from plants transformed with the p35SO2 construct (lanes 3 and 6), plants transformed with the vector pGA643 (lanes 4 and 7 ), and untransformed W38 control plants (lane 5 and 8). Immunodetection was done using polyclonal anti-O2 sera and IgG-conjugated alkaline phosphatase. Molecular mass standards are indicated on the left in kilodaltons. $\mathrm{O} 2$ protein migrates at 65 $\mathrm{kD}$ and is present in $\mathrm{O} 2$ maize endosperm and in p35SO2-transformed tobacco tissues.

\section{References}

Bednarek, S.Y., T.A. Wilkins, J.E. Dombrowski and N.V. Raikhel. Plant Cell 2:1145 (1990).

Broekaert, W.F., H. Lee, A. Kush, N-H. Chua and N.V. Raikhel. Proc. Natl. Acad. Sci. USA 87:7633 (1990).

Chrispeels, M.J. and N.V. Raikhel. Plant Cell 3:1 (1991).

Lerner, D.R. and N.V. Raikhel. Plant Physiol. $91: 124$ (1989).

Varagona, M.J., R.J. Schmidt and N.V. Raikhel. Plant Cell 3:105 (1991).

Wilkins, T.A., S.Y. Bednarek and N.V. Raikhel. Plant Cell 2:301 (1990).

Wright, C.S. J. Mol. Biol. 194:501 (1987). 


\section{REGULATION OF LIPID METABOLISM}

Christopher R. Somerville. Vincent Arondel, Christoph Benning, Clinton Chapple, Linda Danhof, Susan Gibson, Erwin Grill, Ursula Hecht, Yossi Hirschberg, Suzanne Hugly, Koh Iba, Ellen Kearns, Bertrand Lemieux, Yves Poirier, Wolf-Dieter Reiter, Carrie Schneider, John Shanklin, Sharon Thoma, Frank van de Loo and Ruth Wilson

The major research project in the laboratory is a multifaceted investigation of the regulation of plant lipid metabolism. The membrane lipid composition of plants is believed to be an important determinant of tolerance to chilling, and in oilseeds, lipid composition determines the quality of the oll. In both cases one of the most important parameters assoclated with lipid composition is the degree of unsaturation. In order to determine the functional significance of lipid desaturation in chilling stress, anci in order to develop methods for genetic manipulation of desaturase genes in oilseeds, we have isolated and are characterizing a series of mutants of Arabidopsis which are deficient in activity of various enzymes involved in lipid metabolism, in particular, fatty acyl desaturases. The mutants were isolated by screening more than six thousand individuals from an unselected population of mutagenized plants for individuals with alterations in leaf fatty acid composition by gas chromatography (Browse et al., 1985). These mutants have proven very useful for determining the substrate specificities of the various desaturases and have provided new insights into the role of lipid composition in regulating chloroplast biogenesis and function (Browse and Somerville, 1991; Somerville and Browse, 1991). Because the genetic materials developed in this way can be used to generate lines of Arabidopsis with altered membrane lipid composition, we are evaluating the applicability of theories which attribute chilling resistance to membrane lipid composition. We are also using these genetic materials to probe the biochemical mechanisms which regulate lipid metabolism.

Most of our genetic studies have been performed using the small crucifer Arabidopsis thaliana (L.) which is ideally suited as a model plant species for genetics and molecular biology because of its short life cycle, prolific seed production, extensive genetics and the fact that it has the smallest genome known for any higher plant. This feature greatly facilitates the analysis of nuclear genes and can be exploited to permit chromosome walking - something which is currently not possible in other plant species because of the abundance of repetitive DNA and the large genome size. Thus, the organism provides a unique tool as a source of mutants and cloned genes which can be used to identify the homologous genes in crop species by heterologous hybridization.

\section{What determines the amount of membrane?}

One class of mutants (act1) lacks activity for the chloroplast enzyme glycerol-3-phosphate acyltransferase (Kunst et al., 1988). Therefore, these 
mutants are largely unable to synthesize lipids by the plastid $F$ athway which normally provides approximately $50 \%$ of total leaf lipids. As with the orher mutants with alterations in leaf lipid composition, the act1 mutation has relatively minor effects on the growth or appearance of the plants under any conditions (Kunst et al., 1989a). Surprisingly, this mutation also has a relatively slight effect upon the amount of any of the chloroplast or cytoplasmic lipids because the microsomal pathway compensated for the effects of the mutation by a several-fold increase in the rate of microsomal lipid synthesis and in the rate of transfer of lipids from the endoplasmic reticulum to the chloroplast. This compensation revealed the existence of an unsuspected level of metabolic regulation and raised the question as to how the microsomal pathway allocates lipids to a particular membrane.

In order to understand the mechanisms which regulate lipid synthesis and membrane biogenesis it is necessary to know how lipids are transported between the two membranes where most lipid synthesis takes place. One theory is that lipid movement between the endoplasmic reticulum and chloroplast membranes is mediated by a small class of proteins designated as lipid transfer proteins (LTPS). As a first step in a series of experiments to explore the role of these proteins in lipid transfer we cloned the gene for an LTP from spinach leaf by probing a cDNA library with mixed oligonucleotides based on the amino acid sequence of the LTP (Bernhard et al., 1991). We have subsequently used this cDNA to clone the related gene from Arabidopsis. In the course of analyzing the sequence of the spinach LTP CDNA we observed homology to several previously cloned genes which had been considered, on the basis of indirect evidence, to be amylase or protease inhibitors (Bernhard and Somerville, 1989). The high degree of deduced amino acid sequence homology leaves little doubt that the putative amylase inhibitors are in the same protein family as the LTPS.

The sequences of the CDNA clones for the LTPs also revealed the presence of a 27 residue amino-terminal peptide which is not present on the mature polypeptide and which has all of the characteristics of a signal peptide. When the spinach LTP was transcribed and translated in vitro in the presence of dog pancreatic microsomes, the LTP was translocated into the endoplasmic reticulum with concomitant cleavage of the signal peptide (Bernhard et al., 1991). Since there are no known examples of soluble cytoplasmic proteins in which synthesis involves insertion into the endoplasmic reticulum, this observation suggested that the LTP is not a cytoplasmic protein but may be located either outside the cell or in the vacuole. The protein has subsequently been localized outside the cell by immunoelectron microscopy (S. Thoma, unpublished). This location effectively excludes participation of the protein in intracellular lipid transport and raises new questions about the role of the protein. In order to examine the physiological function of the protein we have produced a number of transgenic plants which express antisense copies of the gene. The amount of the protein is strongly reduced in some of these plants and the growth of the plants is reduced. Thus, it is apparent that the protein has a significant role in plant growth. However, we currently have few clues as to what this role may be. 
In view of the fact that the "LTP" proteins cannot be responsible for lipid transfer, we are currently exploring the possibility that the yeast sec14 gene, which encodes a phosphatidylinositol-specific lipid transfer protein, can be used to identify a corresponding protein in Arabidopsis. (Ursula Hecht was supported in part by a fellowship from the Humbolt Foundation; supported in part by grants from the USDA/NSF/DOE Plant Science Center Program and the National Science Foundation.)

\section{Regulation of lipid desaturation}

Mutants are available in Arabidopsis for all of the other known desaturases except for the 18:0-ACP desaturase (e.g., Browse et al., 1985; Kunst et al., 1989b). Analysis of the effects of the corresponding mutations on the fatty acid composition of the various lipids provided the first available evidence concerning the substrate specificity of the enzymes. Similarly, analysis of the effects of these mutations in heterozygotes provided evidence that one copy of an active gene product is generally adequate to obtain wild type fatty acid composition. The mutants had very minor effects on the growth or appearance of plants at standard temperatures. However, both in vivo and in vitro evidence was obtained indicating that decreased unsaturation leads to enhanced thermal stability of the chloroplast membranes at elevated temperatures (Hugly et al., 1989; Kunst et al., $1989 \mathrm{c})$. In the case of the fadB and fadC mutations, which control the activity of a chloroplast $\omega 3$ and an $\omega 6$ desaturase, respectively, this increased thermal stability was correlated with a significantly higher growth rate of the mutants than the wild type at elevated temperature. Thus, these results provide striking evidence that a decrease in lipid unsaturation may be a component of the thermal acclimation response observed in many species of higher plants. Analysis of the effects of the $\mathrm{fad} B$ and fadC mutations on the growth and development of plants at low temperature revealed that tissue which develops at low temperature is chlorotic. The chlorosis is associated with impaired chloroplast development (Fig. 1). We are currently examining the mechanistic basis for this effect. However, it is clear that a high degree of lipid unsaturation is required for normal growth at low temperature.

Recently we extended our search for mutants to the enzymes which control the fatty acid composition of seeds (Lemieux et al., 1990). We have identified mutants deficient in the desaturation of $18: 1$ to $18: 2$, and other mutants deficient in desaturation of 18:2 to $18: 3$ in seeds and roots. We have also identified several mutants deficient in the elongation of $18: 1$ to $20: 1$ and 22:1. These mutations have been genetically mapped and are expected to be very useful in the isolation of the corresponding genes by chromosome walking. 


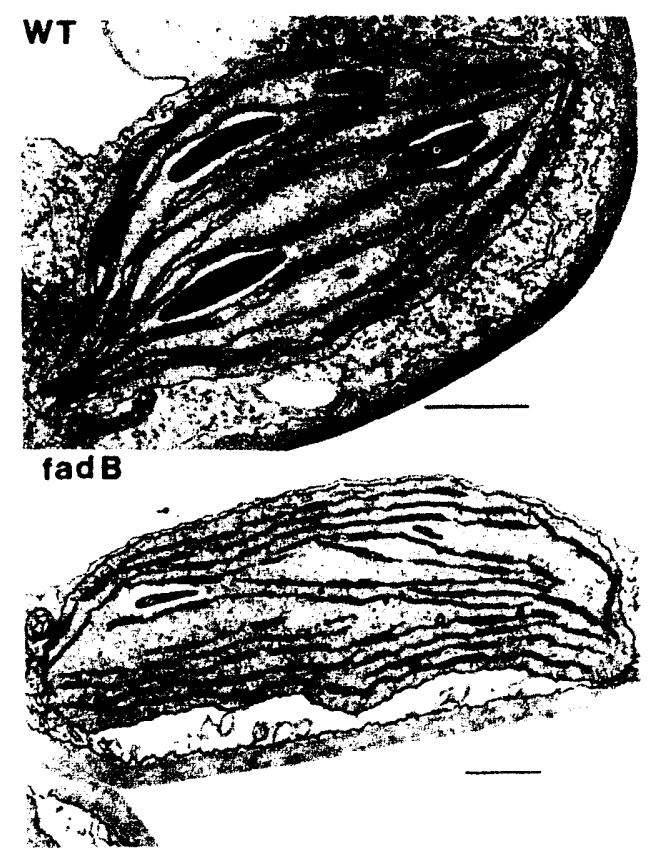

Figure 1. Transmission electron micrographs of chloroplasts from the wild type and an fadB mutant. The chloroplasts were from leaves that had developed during three weeks of growth at $5^{\circ} \mathrm{C}$. Chloroplasts from mutant and wild type tissues grown at $23^{\circ} \mathrm{C}$ were not distinguishable.

Because the current RFLP maps have a relatively low density of mapped RFLPs, it is generally necessary to walk hundreds of kilobases from a mapped RFLP in order to clone most genes. In order to facilitate this, we have constructed a library of the Arabidopsis genome as minichromosomes (YACs) in yeast (Grill and Somerville, 1991). Approximately 1500 clones of 150 to $200 \mathrm{~kb}$ are considered adequate to cover the genome with a high probability. We have isolated more than 10,000 YACs carrying inserts of an average size of $150 \mathrm{~kb}$. A sample of 2,400 YACs, which has been distributed to about 30 other laboratories, has been found to have most of the genome represented. The DNA cloned in these YACs appears to be stable.

We have mapped several loci of interest (i.e., fad2, fad3, fadD, fae1) relative to flanking RFLPs and have initiated the isolation of the YAC clones which carry the DNA between the flanking RFLPs as a prelude to identifying the genes by genetic complementation tests. (Supported in part by a grant from the National Science Foundation; Erwin Grill was supported by a fellowship from the Deutsche Forschungsgemeinschaft; Bertrand Lemieux was supported by an NSERC fellowship; Sue Gibson was supported by an NIH fellowship; Vincent Arondel was supported by an EMBO fellowship.) 


\section{Characterization of enzymes and genes involved in lipid biosynthesis}

As a complementary approach to the genetic work, we have undertaken the characterization of several proteins which are implicated in the regulation of fatty 1acid miodification. In particular, we have purified the stearoyl-ACP desaturase from avocado mesocarp, produced antibodies against the enzyme and used the antibodies: to isolate cDNA clones for the enzyme from castor, cucumber and rapeseed (Shanklin and Somerville, 1991). The castor cDNA was expressed in both yeast and $E$. coli, and functional enzymes were produced in both cases. The very high level of enzyme production in $E$. coli greatly facilitates the production of large quantities of enzyme for biochemical studies. We have used this system to obtain preliminary evidence that the enzyme is an iron-sulfur protein and have initiated a collaboration with Cart Branden and colleagues (Uppsala) to examine the possibility that crystals of the protein can be obtained for X-ray crystallographic studies.

The stearoyl-CoA desaturases in vertebrates utilize cytochrome $b_{5}$ as the electron donor. In order to determine if cytochrome $b_{5}$ is involved in the microsomal desaturase reactions of higher plants we have purified a trypsin-solubilized cytochrome $b_{5}$ from cauliflower microsomal membranes and have prepared antibodies against the cytoplasmic domain. The antibody inhibited $\Delta 12$ desaturation of fatty acids in safflower microsomes (Kearns et al., 1991) providing direct evidence that this desaturase utilizes cytochrome $b_{5}$. This information may facilitate the eventual purification and reconstitution of activity of the microsomal desaturases and identifies a point of potential regulation of fatty acid desaturation.

Ricinoleic acid (12-hydroxyoleic acid) is the major fatty acid found in the oil from castor (Ricinus communis L.). The presence of the hydroxyl group confers useful properties on the oil which has several hundred non-food industrial uses. As a first step in characterizing the enzyme system which introduces the hydroxyl, we have developed methods for the identification of ricinoleic acid by mass spectrometry. This is a prerequisite to the purification of the enzyme because of the presence, in many tissues, of other enzymes which introduce hydroxyl groups at other positions in fatty acids (e.g., $\omega$-hydroxylation). We have also obtained evidence from ${ }^{18} \mathrm{O}_{2}$-labelling studies that molecular oxygen is a substrate for the enzyme. Because the enzymatic activity is labile during purification, we are currentiy exploring genetic methods for identifying a cDNA for the protein which do not depend on prior purification of the enzyme.

The first enzyme in lipid biosynthesis is glycerol-3-phosphate acyltransferase. The properties of this enzyme are implicated as the determinative factor in one of the current theories of chilling sensitivity ("the Murata hypothesis") and are thought to regulate the quality of cocoa butter and other high value lipids. Murata and colleagues have recently cloned a cDNA for this enzyme from squash. We have used their sequence to isolate corresponding CDNA clones from Arabidopsis (chilling resistant) and squash (chilling sensitive). These genes are currently 
being introduced into the Arabidopsis mutant (act1) which lacks activity for this enzyme in order to directly test the importance of variation in the kind or amount of this enzyme on chilling sensitivity. (Carrie Schneider was supported in part by an NSF Postdoctoral Fellowship; Ellen Kearns was supported in part by an MSUBiotechnology Fellowship; supported in part by grants from the USDA/NSF/DOE Piant Science Center Program and a gift from Pioneer Hybrid International.)

\section{Production of biodegradable thermoplastic in higher plants}

Poly- $\beta$-hydroxybutyrate (PHB) is an aliphatic polyester produced as a carbon storage reserve in numerous species of bacteria such as Alcaligenes eutrophus. In these bacteria, PHB accumulates as granules of $100-800 \mathrm{~nm}$ in diameter which appear to be surrounded by a half unit membrane approximately $3 \mathrm{~nm}$ thick. Under certain conditions, PHB may comprise more than $80 \%$ of the cell mass. PHB has attracted interest as a potential biodegradable thermoplastic. At present, attention is focused on the production of PHB and related compounds in large scale fermentation facilities. However, the projected cost of production would make PHB too expensive for many potential applications. Therefore, we are exploring the possibility that PHB can be produced in high yield and at low cost by transferring the genes involved in FHB synthesis in $A$. eutrophus to a suitable higher plant.

PHB is synthesized in bacteria by the sequential action of three enzymes which catalyze the conversion of acetyl-CoA to acetoacetyl-CoA, then to hydroxybutyrylCOA, then to PHB. The genes encoding the three enzymes have been cloned from $A$. eutrophus. Expression of the $A$. eutrophus genes in $E$. coli resulted in production of $\mathrm{PHB}$ granules identical to those found in $A$. eutrophus, indicating that no other proteins are specifically required (Slater et al., 1988).

Our short term goal is to determine if we can produce PHB in a plant cell. Our collaborator Douglas Dennis (James Madison University) has provided us with the PHB operon from $A$. eutrophus. We have subcloned the three genes into derivatives of the binary Ti plasmid pBIN19. The genes have been placed distal to the constitutive $35 \mathrm{~S}$ promoter from cauliflower mosaic virus and introduced into a non-oncogenic strain of Agrobacterium tumefaciens. These constructs have been used to initiate the production of transgenic plants by established methods. If these preliminary experiments indicate that PHB can be produced in plant cells, the long term goal will be to divert the carbon which is normally stored as starch or sucrose into PHB. (Yves Poirier was supported in part by a fellowship from NSERC; the work was supported in part by grant \#DMB-9014037 from the SGER program at NSF.)

\section{The pathway of sulfolipid biosynthesis}

One of the unique constituents of chloroplast membranes is sulfolipid. Although the structure of this lipid was determined more than 20 years ago, the 
biosynthetic pathway has not been determined and the functional significance of this membrane component has not been determined. The purple photosynthetic bacterium Rhodobacter sphaeroides also accumulates substantial amounts of sulfolipid. Mutants which do not contain sulfolipid were identified by screening a mutagenized population by thin layer chromatography of lipid extracts from several thousand independent colonies. These mutants retain the capacity to grow photoautotrophically and several of them accumulate putative precursors of sulfolipid. Determination of the structures of the accumulated compounds may permit inferences to be made about the steps in the pathway.

The genes which complement three of the mutants were isolated by complementing the mutants with a cosmid library. The cloned genes will permit the creation of mutants, by gene disruption techniques, which completely lack sulfolipid. Two of the genes are very closely linked and are probably cotranscribed. Other genes of the pathway may also be in the same operons, and it may be possible to create mutations in additional steps of the pathway by gene disruption methods. In a series of complementary experiments we have found that cell-free extracts of the bacterium are capable of synthesizing sulfolipid from exogenous phosphoadenosine phosphosulfate. In vitro synthesis has not previously been possible with any other system and should greatly facilitate the testing of hypotheses concerning the reactions involved in sulfolipid biosynthesis. (Supported in part by a grant from the USDA/NSF/DOE Plant Science Center Program.)

\section{Chilling sensitive mutants of Arabidopsis}

The effective geographical distribution of many species of plants is limited by the sensitivity of these plants to "chilling temperatures" which do not adversely affect other species. The physiological characteristics which are responsible for chilling sensitivity or resistance are not known. Attempts to identify relevant biochemical differences between chilling-sensitive and chilling-resistant species are confounded by the many irrelevant differences (Graham and Patterson, 1982). We are interested in the possibility that chilling-resistant species have gene products which are not required for growth in temperate conditions but which are required for survival at low temperature. In order to test this hypothesis we have isolated several mutants which transform the chilling-resistant species Arabidopsis thaliana to an extremely chilling-sensitive phenotype. The mutants were isolated by growing populations of mutagenized plants at permissive temperature until they were established, and then shifting the plants to chilling temperature to test for chilling injury. We currently have several lines which are normal in appearance when grown at $23^{\circ} \mathrm{C}$ but turn chlorotic or necrotic after several days of exposure to temperatures in the range of about $13^{\circ} \mathrm{C}$. Analysis of one line, designated PM11, indicated that chilling sensitivity in this line is due to a single recessive nuclear mutation at a locus we designate chs1. In addition to chilling-induced chlorosis, this line exhibits massive lon leakage following 24 to $48 \mathrm{~h}$ of chilling at $13^{\circ} \mathrm{C}$. Detailed metabolic tracer studies of the mutant have 
revealed only one metabolic alteration, a change in the amount of label in steryl esters (Hugly et al., 1990). Although it has not as yet been possible to determine the precise biochernical lesion responsible for this effect, the results suggest either that variation in steryl esters may cause chilling sensitivity, or that this aspect of metabolism is uniquely sensitive to changes in cell structure or function which precede the onset of visible signs of chilling injury.

In order to determine how many different kinds of mutations can give rise to chilling sensitivity, we have isolated a large number of additional mutants with this phenotype. Several of these mutants carry additional mutant alleles of the chs 1 locus indicating that the mutation in PM11 is not a rare event. Thus, it is possible that the chs 1 gene product is specifically required for growth at low temperature. We are currently placing the other mutants of this type into genetic complementation groups in order to obtain an insight into the biochemical complexity of the phenomenon. (Supported in part by a grant from the USDA-CRGO \#USDA-87-CRCR-1-2507; Carrie Schneider was supported by an NSF Postdoctoral Fellowship.)

\section{References}

Bernhard, W. and C.R. Somerville. Arch. Biochem. Biophys. 269:695 (1989).

Bernhard, W.R., S. Thoma, J. Botella and C.R. Somerville. Plant Physiol. 95:164 (1991).

Browse, J., P. McCourt and C.R. Somerville, Science 227:763 (1985).

Browse, J., L. Kunst, S. Anderson, S. Hugly and C.R. Somerville, Plant Phy'siol. 90:522 (1989).

Browse, J. and C.R. Somerville. Ann. Rev. Plant Physiol. Molec. Biol. 42:467 (1991).

Graham, D. and B.D. Patterson. Ann. Rev. Plant Physiol. 33:347 (1982).

Grill, E and C.R. Somerville, Molec. Gen. Genet. in press.

Hugly, S., L. Kunst, J. Browse and C.R. Somerville. Plant Physiol. 90:1134 (1989).

Hugly, S., P. McCcurt, J. Browse, G.W. Patterson and C.R. Sornerville. Plant Physiol. 93:1053 (1990).

Kearns, E.V., S. Hugly and C.R. Somerville. Arch. Eiochem. Biophys. 284:431 (1991)

Kunst, L., J. Browse and C R Somerville. Proc. Natl Acad. Sci. USA 85:4143 (1988).

Kunst, L., J. Browse and C.R. Somerville. Plant Physiol. 90:846 (1989a).

Kunst, L., J. Browse and C.R. Somerville. Plant Physiol. 90:943 (1989b)

Kunst, L., J. Browse and C.R. Somerville. Plant Physiol. $91: 401$ (1989c).

Lemieux, B., M. Miquel, C.R. Somenville and J. Browse. Theor. Appl. Geriet. $80: 234(1990)$.

Shanklin, J. and C.R. Somerville. Proc. Natl. Acad. Sci. USA, in press.

Slater, S.C. Voige, W.H. and D.E. Dennis. J Bacteriol. 170:4431 (1988).

Somerville. C.R. and J. Browse. Science, 251:1 (1991) 


\section{MOLECULAR BASES OF PLANT DISEASE RESISTANCE MECHANISMS}

Shauna Somerville. Lisa Carlson, Kent Kerby, Ke-Bin Liu, Sam Simons, Bernice Slutsky, Jun Tsuji and Jennifer Walsh-Weller

Genes which confer resistance to specific races of fungal pathogens have been identified in a broad range of plant species and have been important in the development of modern cultivars of many crops. Because relatively few genes mediate race-cultivar specific resistance and these genes are of economic significance, disease resistance genes represent attractive targets for genetic engineering (Kleese and Duvick, 1980). However, in spite of extensive genetic and cytological investigations of a number of host-pathogen systems, virtually nothing is known about the products of resistance genes. Furthermore, because the biochemical events of disease development are poorly described, rational approaches to chemical plant protection are precluded. Our long term goal is to develop a description of the molecular and biochemical events associated with disease development and with the expression of resistance. We have chosen to work with two complementary host-pathogen systems. One is the powdery mildew disease of barley caused by the fungal pathogen Erysiphe graminis $\mathrm{f}$. sp. hordei and the other is the interaction of Arabidopsis thaliana with the bacterial pathogens Xanthomonas campestris pv. campestris and Pseudomonas syringae pv. syringae.

One specific objective is to identify and characterize the gene(s) of the barley MIa powdery mildew resistance locus. The characterization of an MI-a gene product will be an important step in determining a key biochemical component of disease resistance. In addition, understanding the genetic mechanism that is responsible for the highly polymorphic nature of the MI-a locus is of both practical and theoretical interest, since the genetic structure of this locus will dictate the range of novel resistance alleles that may be created in vitro for the purposes of genetically engineering disease resistance. Arabidopsis is a model plant species that offers many technical advantages for isolating genes, such as disease resistance genes, for which we lack biochemical information (Meyerowitz, 1987). A second objective of our research program is to clone those genes of Arabidopsis that mediate resistance to $X$. c. campestris, the causal agent of the black rot disease, and those genes that regulate responses to non-host pathogens like $P$. $s$. syringae. These genes will then be used as tools in characterizing host resistance mechanisms.

\section{Barley mutants with aftered disease reaction phenotypes}

Host-pathogen interactions in the powdery mildew disease are characterized by a high degree of specificity that is typical of interactions described by the 
"gene-for-gene" hypothesis (Ellingboe, 1972; Moseman, 1966). We have utilized mutational analysis to study the race-cultivar specific interactions of the barley powdery mildew disease. Barley line $\mathrm{Cl}-16137$ (AlgR) carrying the $\mathrm{Ml}$-a 1 resistance allele (Moseman, 1972), was treated either with sodium azide or by $\gamma$-irradiation. A total of approximately $118,000 \mathrm{M} 2$ seedlings derived from the mutagenized seed were screened for an altered phenotype when inoculated with race CR3 of $E$. $g$. hordei. Forty-two mutants were recovered; 23 of the mutants are fully susceptible to E. g. hordel, race CR3 and 19 mutants have intermediate levels of susceptibility. AlgR, the parental line, is fully resistant or immune to race CR3. For each of the mutants, the rachilla type and the hordein protein pattern were determined in order to confirm that the line was a true mutant and not a contaminant. No fully susceptible mutants were recovered from populations of M2 seedlings derived from sodium azide mutagenesis.

Based on these preliminary results, we suggest that either the MI-a locus is genetically complex or that resistance is conditioned by more than one gene. If the latter explanation is true, then the gene products must act in series to mediate the extreme form of resistance observed in immune lines like AlgR, and these genes are closely linked. It is possible that $\gamma$-irradiation, but not sodium azide mutagenesis, is sufficiently damaging to the $M I-a$ allele found in AlgR to fully disrupt expression of the $M /$-a locus resulting in highly susceptible mutants. We plan to test the lattei hypothesis by complementation analyses among the 42 mutants.

\section{Molecular markers associated with the MLa encoded resistance}

The congenic bartey lines AlgR and AlgS differ by the respective presence or absence of the dominant MI-a 1 resistance allele (Moseman, 1972). We examined the protein profiles of AlgR and AlgS by 2-D PAGE (Granier, 1988; O'Farrell, 1975). Two constitutive differences between the barley isolines were observed in healthy, uninoculated tissue. A set of polypeptides of 37-40 kD and with an apparent $\mathrm{pl}$ of 6.3 were observed in the protein profile of AlgS but were absent in the resistant isoline. In addition, a polypeptide of $\mathrm{pl} 6.7$ was identified that was $101 \mathrm{kD}$ in AlgR and $103 \mathrm{kD}$ in AlgS. The difference in mobility of the latter polypeptide was verified by the presence of both the $101 \mathrm{kD}$ and $103 \mathrm{kD}$ polypeptides in the protein profile of the $F_{1}$ [AlgR $\times$ AlgS]. Since these barley lines are related by 18 generations of crossing, any polymorphisms that are found are likely to be at or closely linked to the MI-a locus.

In order to determine whether either of these polymorphic polypeptides is likely to be an Ml-a gene product, the protein profiles of additional barley genotypes were examined. In a mutant of AlgR with increased susceptibility to $E$. g. hordei, the $101 \mathrm{kD}$ polypeptide displayed a $63 \%$ reduction in the intensity of labelling with ${ }^{35} \mathrm{~S}$-methionine + cysteine when quantitated with the Bioimage 110 Image Analyzer. Additionally, we found that a polymorphism for this polypeptide ( $p / 6.8,101 \mathrm{kD}$ ) 
existed in the susceptible cultivar Siri. The Siri form of the polypeptide was replaced by the AlgR form in the line S-01, which was derived by backcrossing the MI-a1 resistance allele from AlgR into Siri (Køster and Stølen, 1987). This correlation between resistance to race CR3 of E. g. hordei and the presence of the $101 \mathrm{kD}, \mathrm{pl} 6.7$ polypeptide did not hold in two further results. A second partially susceptible mutant derived from AlgR, M332, displayed normal levels of the $101 \mathrm{kD}$ polypeptide. Also, the barley line P-01, which was generated by backcrossing the Ml-a1 allele into Pallas (Køster et al., 1986), did not exhibit the AlgR form of the polymorphic polypeptide. Based on these results, we concluded that protein polymorphisms associated with the MI-a 1 allele could not be detected in 2D-PAGE profiles with the methods we were using.

Taking advantage of the fact that AlgR and AlgS are congenic lines, we are attempting to make a subtractive cDNA library of uninoculated AlgR coleoptile tissue (Akowitz and Manuelidis, 1989). Assuming the two isolines are $>99 \%$ similar (Moseman, 1972) and barley, like wheat, expresses about 30,000 different mRNA species, then the number of mRNA species that are recovered in the subtractive cDNA library should be $<300$. In fact, this number is expected to be substantially less than 300 mRNA species because few of the genes in the introgressed region of the genome are expected to exhibit substantial differences in amount or sequence between AlgR and AlgS. To date, the CDNA populations have been constructed and are being amplified via the polymerase chain reaction to levels sufficient to drive renaturation reactions for the subtractlve part of the experiment. Once the subtracted cDNA library has been established, the cDNA's will be tested for co-segregation with the resistance allele in $300 \mathrm{~F}_{3}$ [AlgR $\times$ AlgS] families. By this means, we hope to identify one or a limited number of cDNA's that are unique to AlgR and altered or missing in susceptible barley genotypes.

\section{Peroxidases: Biochemical markers of infection}

Plant peroxidases increase during some infections, and as a result, have been implicated in a number of host defense responses (Hammerschmidt and Kuc, 1982; Hammerschmidt et al., 1982; Holden and Rohringer, 1985; Vance et al., 1980). Notably, wall peroxidases are responsible for initiating the polymerization of hydroxy-cinnamyl alcohol into lignin-like structures (Vance et al., 1980). We wish to address the arguments that peroxidases play a role in host-pathogen interactions and possibly in host resistance mechanisms by determining the localization and timing of occurrence of specific peroxidases during the infection sequence in compatible and incompatible interactions between barley and $E$. $g$. hordei. A more detailed knowledge of this induction process will lead to a better understanding of the molecular events that occur in host-pathogen interactions and will help to distinguish infection-specific responses from general stress responses. 
The intercellular wash fluid of barley leaves contains at least seven peroxidase isozymes (rerby and somerville 1987). Two of these isozymes with pl's of 85/8.6 and 5.2 increase in response to infection by $E$. g. hordei. The relative specific activity of the pi 7.7 isozyme does not change in response to inoculation with $E g$ hordet, and will be used as a control in future experiments. The pl $8.5,8.6$ isozyme has been purified to homogeneity by chromatcgraphy on caiboxy-methy! cellulose isoelectric focusing in a Rotofor apparatus (BioRad). and gel filtration chromatography on a Sephadex G75 matrix. At present, methods for purifying the pl 5.2 and $p / 7$ peroxidase isozymes are being developed. Once the extracellular peroxidases have been characterized, the localization and timing of induction of the infection-related peroxidases will be compared to such cytological responses as papillae formation and the deposition of phenolic compounds in the cell wall adlacent to penetration sites

\section{Synthesis of a phytoalexin in Aratiofopsis}

Previously (Tsuji at al, 19(x)), leaves of Arabchopsis were found to accumulate a phiytoalexin following inoculation with P. S. syringae. About $2.5 \mathrm{mg}$ of the Arabidopsis phytalexin, which wh have narred arawilexin, were purified from 1.28 $\mathrm{ng}$ tresh weight of leat tissue by a combination of reverse phase flash chromatography, TLC, and reverse phase HPLC. A molar extinction coefficient of $14.800 \mathrm{M}^{\circ} \mathrm{cm}$ was calculated for the purified phutoalexin using the BeerLamoen lav. This extinction coefficient was then used to quantitate the accurnuation of arabilexin In loaves inoculated with $P$. S syringae, arabilexin accumulated within 12 hours post-inoculation and reached a maximum level of approxmately $8 / .9 g^{\prime}$ resh weight by 36 hours. The accumulation of arabilexin was hegatively correlated with the growth in planta of $P$. s. syringae. In addition, the in wiro growth of both the fingus ckaciosporium cucumerinum and the adtem $P$ s syrngae was inhimed by arabilexin.

\section{References}

Akowtz A and L Manuelidis Gene 81295 (1989)

Filingloe. A. Phylopath. 62 401 (197\%)

Granter F Electrophoresis $9712(1988)$

Hammenschmid $A$ and I Kur Plysiol Plant Pathol 2061 (1982)

Hammerschmid? R. E.M Nuckles and J. Kuc. Physiol Plant Pathol 2073 (1982).

Holdat. OW and Fonringer flant Physiol. 70820 (1985).

Kerty, Kand SC. Somenville. Physiol. Mol Plant Pathol 35:323 (1989).

heese FA and DN Duvick In Geneit Improvement of Crops: Emergent

rochnques (1 Rubenstein, B Gegenbach R I. Phillips, E. Green, eds)

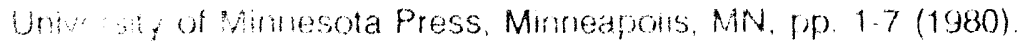

Kol P and Stolen Plant Brew 9879 (1987)

Kowter P. L Wirk O Stulen and J Lohde Crop Sci '26903 (1366)

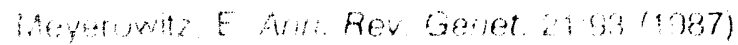


Moseman, J.G. Ann. Rev. Phytopathol. 4:269 (1966).

Moseman, J.G. Crop Sci. 12:681 (1972).

O'Farrell, P.H. J. Biol. Chem. 250:4007 (1975).

Tsuji, J., R. Hammerschmidt and S.C. Somerville. Phytopath. 80:1054 (1990).

Vance, C.P., T.K. Kirk and R.T. Sherwood. Ann. Rev. Phytopathol. 18:259 (1980). 


\section{BIOCHEMICAL AND MOLECULAR ASPECTS OF PLANT PATHOGENESIS}

Junathan D. Walton. Frank Holden, Robert Meeley, Daniel Panaccione, Holly Schaeffer and John Scott-Craig

The interactions between a plant and a pathogen are controlled by manifold structural, chemical, and biochemical features of the two organisms. The plant has both pre-formed and induced mechanisms to limit invasion. The pathogen must penetrate, overcome the host defenses, and obtain nutrients. Cell wall degrading enzymes are examples of general pathogenicity factors. All fungi make them, and indirect evidence suggests that in some cases they are required for penetration and nutrient acquisition. Such factors, however, do not account for the exquisite specificity frequently present in host-pathogen interactions, in which compatibility and incompatibility are determined by single genes in the pathogen and single genes in the host. Host-selective toxins are low molecular weight compounds produced by some pathogenic fungi. A fungus that makes a hostselective toxin is pathogenic on just those plants that are sensitive to the toxin, and, conversely, isolates of the fungus that do not make the toxin are not pathogenic on those plants. Host-selective toxins are among the very few molecular agents of plant disease specificity known.

This research group is studying the biochemistry and molecular biology of representative examples of general and specific pathogenicity factors. Both types of studies are being done with an important fungal pathogen of cereals, Cochliobolus (Helminthosporium).

\section{Cloning of the Toxe locus from C. carbonum}

Host-selective toxins have been the critical factors in two major epidemics of crop plants in the U.S. in the past fifty years, including the Southern Corn Leaf Blight epidemic of 1970 (Walton, 1991). Host-selective toxins are also critical determinants of other diseases caused mainly by other species of Cochliobolus and by species of Alternaria. We have chosen the leaf spot disease of maize, caused by Cochliobolus carbonum, as a model toxin system. C. carbonum produces a host-selective toxin called HC-toxin, which is a cyclic tetrapeptide of structure cyclo(D-Pro-L-Ala-D-Ala-L-AOE), where AOE stands for 2-amino-8-oxo9,10-epoxidecanoic acid. The toxin and the producing fungus affect only maize that is homozygous recessive at the nuclear $\mathrm{Hm}$ locus. Production of HC-toxin is controlled by a single Mendelian gene in C. carbonum called Tox2. Interestingly, production of the chemically unrelated host-selective toxins, T-toxin and victorin, made by $C$. heterostrophus (Helminthosporium maydis) race $T$ and C. victoriae, respectively, are also controlled by single, but different, genes. These other genes are called Tox 1 and Tox3, respectively. Our work is aimed at understanding, first, how single genetic loci can control production of complex secondary metabolites such as toxins, and second, the evolution of Cochliobolus, 
especially the apparent rapid appearance of new toxicogenic races and species (Loonard, 1973).

We reported the Identffication, purfication, and characterization of two enzymes Involved in the blosynthesis of HC-toxin (Walton, 1987; Walton and Holden, 1988). These enzymes, HTS-1 and HTS-2, catalyze activation and epimerization of Lproline and L-alanine, respectively. Both acthities are linked to the Tox2 locus and are lacking in isolates of the fungus that do not make HC-toxin (Walton, 1987). Using antibodies and oligonucleotide probes based on the amino acid sequence of the enzyme we have identified the gene for HTS-1. The entire gene as well as flanking DNA totalling $22 \mathrm{~kb}$ is lacking in all tested isolates of C. carbonum that do not make HC-toxin. The presence of this 22-kb region of DNA genetically co-segregates with HC-toxin production. This result indicates that HCtoxin production did not evolve by a simple mutation or by a genetic rearrangement.

It has been proposed, but never experimentally verified, that genes can move between unrelated species, a process called horizontal gene transfer (Lewin, 1982). For several theoretical reasons, we feel that this may have occurred in the evolution of $C$. carbonum. First, three other fungi unrelated to $C$. carbonum produce compounds closely related to HC-toxin (Walton, 1990). Since secondary metabolites are often complex (as are HC-toxin and its analogs), and their biosynthetic enzymes specialized (as are HTS-1 and HTS-2), the existence of particular secondary metabolites in unrelated organisms has led Luckner (1984) and others (Kleinkauf and von Döhren, 1990) to propose horizontal gene transfer as an explanation. Second, if such transfer can occur, movement of single "genes" such as Tox2 is much more likely than the movement of multiple, unlinked genes. Third, a plausible mechanism exists. Fungal hyphae, even of vegetatively incompatible strains, can fuse (form anastamoses) for long enough for the transfer of DNA to occur (Collins and Saville, 1990). We are beginning our examination of the hypothesis that $C$. carbonum acquired the capability to make HC-toxin by horizontal gene transfer by using our cloned Tox2 DNA to search for related DNA in the fungi that make compounds related to $\mathrm{HC}$-toxin.

Our analysis of the $22 \mathrm{~kb}$ of Tox2-unique DNA has revealed that it is more complicated than originally expected. Portions, and perhaps all of it, are present in more than one copy in the genome of tox ${ }^{+}$isolates. At one end of the $22 \mathrm{~kb}-$ unique region there are restriction fragment length polymorphisms (RFLP's) indicating that some of the $22 \mathrm{~kb}$ of DNA is present in at least four copies. At the other end of the $22 \mathrm{~kb}$-unique region, where the gene for HTS-1 lies, there is also an RFLP, but only with the enzyme Apal. The presence of multiple coples complicates efforts to convert a tox ${ }^{+}$isolate to a tox isolate by transformationmediated gene disruption. We are creating isolates of $C$. carbonum that are disrupted in one or the other copy of the gene for HTS-1 using different selectable 
markers and will attempt to create a strain disrupted in both copies by conventional crossing.

Repeated attempts to detect mRNA's derived from the 22-kb region by Northern blotting have failed, presumably because the messages are rare and very large (5 to $6 \mathrm{~kb}$ ), and hence difficult to isolate intact. By screening a cDNA library with DNA probes derived from the 22-kb region we have identified at least two and perhaps three additional transcribed regions. By expressing these cDNA's and the corresponding genomic clones in a prokaryotic expression vector, followed by screening with our anti-HTS-2 antiserum, we will determine if the gene for HTS2 is part of the $22 \mathrm{~kb}$.

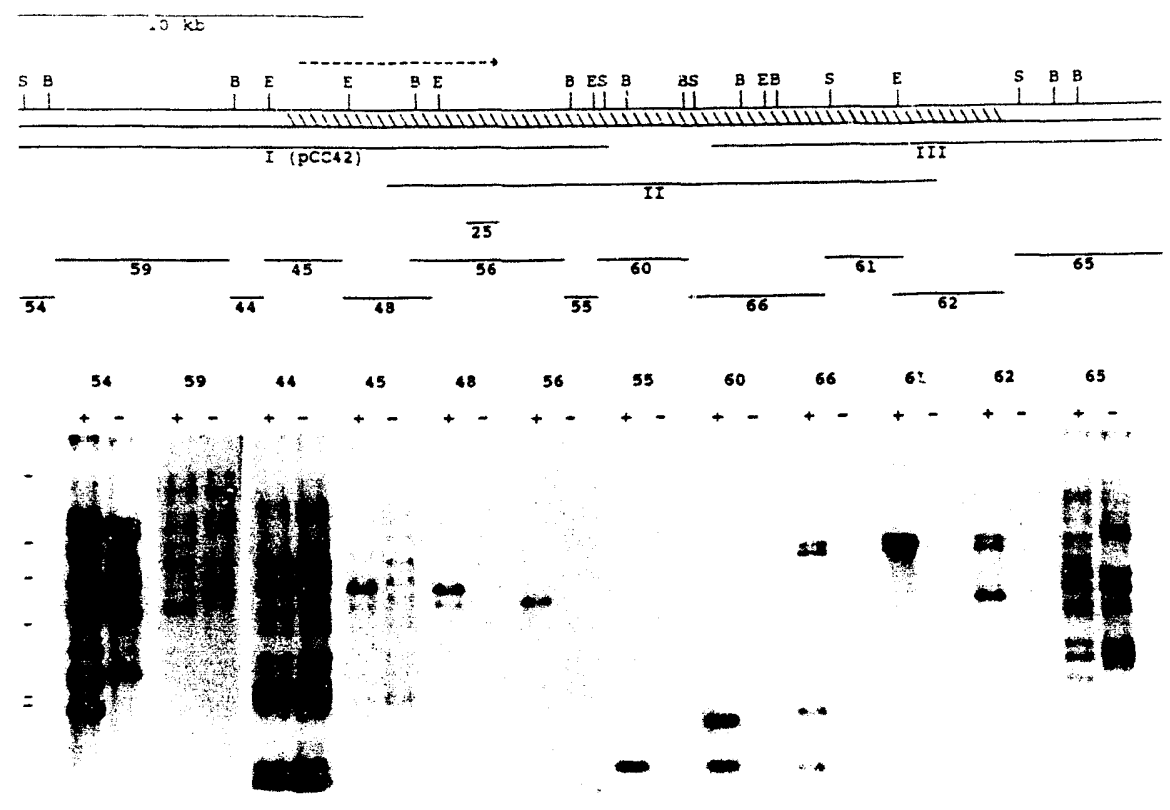

Figure 1. Analysis of the $22 \mathrm{~kb}$ of DNA that is associated with HC-toxin production in $C$. carbonum race 1 On top is shown the restriction map of the $C$. carbonum chromosome, based on three overlapping lambda clones, shown below. The hatched area indicates DNA unique to race 1 (tox $\left.{ }^{+}\right)$isolates. The dashed arrow shows the presumptive location of the gene for HTS-1, based on its partial CDNA cione ( $p c c 25)$. Underneath the map are shown numbered subcloned fragments of the 22-kb region. These fragments were used as radiolabelled probes against DNA blots of total genomic DNA from a tox ${ }^{+}$and a tox isolate, which are shown at the bottom of the figure. Lines at the left of the DNA blots indicate lambda/Hindlll size markers. 
If the 22-kb region exists as two coples in the genome of tox ${ }^{+}$C. carbonum isolates, and if both are functional, then the genetics indicates that they must be linked. However, attempts to walk along the chromosome from one copy to the next have been frustrated by the presence of repeated DNA flanking the $22 \mathrm{~kb}$. Using overlapping genomic clones in phage lambda EMBL3, we have walked 15 $\mathrm{kb}$ from one end of the Tox2-unique DNA and $10 \mathrm{~kb}$ from the other. All of this flanking DNA that we have examined is repeated 10 to 30 times and is present in both tox and tox isolates. However, there are Tox2-linked RFLP's in this repeated DNA, which can be used, albeit with difficulty, to walk through the repeated DNA. Furthermore, the presence of Tox2-linked RFLP's in this repeated DNA suggests that the Tox2 locus in the broader sense consists of both unique and repeated DNA. (Supported, in part, by NSF grant \#87-15608 and the MSU Biotechnology Research Center.)

\section{Metabolism of HC-toxin by maize as the basis of host-selectivity}

Resistance to $C$. carbonum race 1 (tox ${ }^{+}$) and to $\mathrm{HC}$-toxin in maize is controlled by a single dominant Mendelian gene. HC-toxin contains an epoxide group which is required for activity, and plants are known to contain epoxide hydrolases. We have been testing the possibility that the $\mathrm{Hm}$ locus encodes or controls expression of an enzyme that metabolizes $\mathrm{HC}$-toxin to a non-toxic derivative, such as the hydrolyzed epoxide. Radiolabelled HC-toxin was prepared by feeding $\left[^{3} \mathrm{H}\right] \mathrm{D}$-alanine to the fungus. Tritiated HC-toxin was fed to maize leaves and the resulting products analyzed by reverse phase HPLC and an in-line radioactivity detector. Maize leaves were able to metabolize HC-toxin very effectively to a single product. Contrary to our expectations, this was not the diol formed by hydrolysis of the 9,10-epoxide, but rather the 8-alcohol formed by reduction of the 8-ketone (Meeley and Walton, unpublished results). This compound is non-toxic (Kim et al., 1987).

When tritiated HC-toxin is fed to leaves through the transpiration stream, there are no significant differences in rate or extent of $\mathrm{HC}$-toxin reduction by resistant and sensitive plants. This originally suggested that detoxification of HC-toxin is not the basis of its host-selectivity. Nonetheless, in order to study an apparently novel enzyme and to see if the enzymes from resistant and susceptible maize differed in any way (e.g., in $\mathrm{K}_{\mathrm{m}}$ or $\mathrm{V}_{\max }$ ), we have developed an in vitro assay for HC-toxin reduction. "HC-toxin reductase" - STR) can be purified from etiolated maize tissue by ammonium sulfate precipitu..on, gel filtration, and ion exchange chromatography. It requires NADPH and is inhibited by p-hydroxymercuribenzoate. Surprisingly, however, despite numerous attempts, we have been able to find the enzyme only in resistant maize (inbred Pr1) and not in susceptible maize (inbreds $\mathrm{Pr}$ and $\mathrm{K} 61$ ). Host-selective reaction to $\mathrm{C}$. carbonum race 1 (tox ${ }^{+}$) can be assayed by inoculating single etiolated mesocotyls (Heim et al., 1983) and we have developed an assay for HCTR in single etiolated shoots. Therefore, individual plants can be tested for reaction to $C$. carbonum race 1 and 
presence of HCTR. Currently we are testing the genetic linkage between resistance to $C$. carbonum race 1 and presence of extractable HCTR. In collaboration with Steve Briggs at Pioneer Hi-Bred International, we are assaying HCTR in a variety of experimental maize lines that have either spontaneous or transposon-induced mutations to sensitivity at the $\mathrm{Hm}$ locus, as well as some lines that have reverted to $\mathrm{HC}$-toxin resistance due to excision of the transposable element. Our studies in combination with Briggs' work on the cloning of the $\mathrm{Hm}$ locus may, for the first time, lead to a complete genetic, molecular, and biochemical understanding of a plant disease interaction. (Supported, in part, by the MSU Research Excellence Fund and an MSU All-University Research Initiation Grant.)

The role of polygalacturonase and other cell wall-degrading enzymes in plant disease

Endo-polygalacturonase (endoPG) is an enzyme made by all cellular plant pathogens (Collmer and Keen, 1986). Some studies indicate a function for this enzyme in triggering plant defense responses, in addition to its role in degrading the pectinaceous middle lamella during penetration (Cervone et al., 1989). As assayed in culture filtrates, endoPG activity is induced by pectin and suppressed by sucrose. C. carbonum makes a single endoPG enzyme (Walton and Cervone, 1990). It does not make detectable pectic lyase. In order to evaluate definitively the role of endoPG in disease, we cloned the gene using oligonucleotides based on $\mathrm{N}$-terminal and tryptic peptide amino acid sequences in the polymerase chain reaction (PCR). To the best of our knowledge this is the first endoPG gene to be cloned from a plant pathogenic fungus (Scott-Craig et al., 1990). The endoPG gene from $C$. carbonum has several significant regions of amino acid identity to the endoPGs from bacteria and tomato. In $C$. carbonum, integration of transforming DNA is almost always homologous, which allowed us to construct a specific endoPG-minus strain of the fungus using transformation-mediated gene disruption. The resulting mutant lacked endoPG activity but could still grow on pectin as sole carbon source and was still pathogenic (Scott-Craig et al., 1990). The mutant still made exoPG, which probably explains its ability to grow on pectin. Whether the exoPG can account for the ability of the mutant to grow on pectin and to cause disease is currently under investigation.

We are also investigating the role of other cell wall-degrading enzymes (CWDE's) in disease. Two xylanase isozymes have been purified from culture filtrates of $C$. carbonum and partially sequenced (Holden and Walton, unpublished results). The enzymes have pl's greater than 9.5 and have molecular weights of less than $20 \mathrm{kDa}$. Cloning of the corresponding genes using oligonucleotiae probes is in progress.

C. carbonum, as well as $C$. heterostrophus (Anderson, 1978), makes laminarinase, also known as B1,3-glucanase. We have detected in culture filtrates of $C$. 
carbonum a single enzyme capable of degrading laminarin and have purified it to homogeneity. The enzyme has a molecular weight of $63 \mathrm{kDa}$ and a pl of 6.4 (Van Hoof and Walton, unpublished results). Unlike higher plant B1,3-glucanases that are induced in response to stress such as pathogenic attack, it is an exoacting enzyme. (Supported, in part, by a NATO Grant for International Collaboration, the MSU Research Excellence Fund, and a Biomedical Research Support Grant.)

\section{Signal transduction of a host-specific elicitor/toxin}

Victorin is a chlorinated host-specific cyclic peptide made by the oat pathogen C. victoriae (Wolpert et al., 1985). It is active at picomolar concentrations as a toxin and as an elicitor of phytoalexins (Mayama et al., 1986). Victorin induces protoplasts to make an extracellular glucan which we have identified as callose by monomer composition and linkage analyses (Schaeffer and Walton, 1990). Callose biosynthesis is a common response of many plants to environmental stress including pathogen invasion. Using protoplasts as the system, victorin as the trigger, and incorporation of $\left[^{14} \mathrm{C}\right]$ glucose into callose as the quantifiable response, we are studying the poorly understood processes by which plant cells respond to environmentally-induced stress. The use of protoplasts insures that all cells perceive the signal simultaneously and allows cell death to be monitored. Protoplasts begin to make callose in response to victorin only after a lag of one hour, and both cell death and callose synthesis are blocked by pretreatment with inhibitors of protein and RNA synthesis. Aluminum, a phytotoxic heavy metal implicated in acid rain-induced forest dieback, also induces oat protoplasts to make callose. Aluminum is active only at much higher concentrations than victorin and is non-selective. Pretreatment with an inhibitor of protein synthesis blocks callose synthesis in response to aluminum but does not prevent cell collapse (Schaeffer and Walton, 1990). These results indicate that although there are similarities in the ways in which plant cells transduce these biotic and abiotic stresses, there are also important differences.

The best current hypothesis about how callose synthesis is activated is that injury or other stress damages the plasma membrane and allows calcium ion concentrations in the cytoplasm to rise. Since callose synthase requires calcium (Kauss, 1987), this could trigger callose production. However, several of our results are inconsistent with such an activation mechanism. First, cells make callose in response to aluminum and victorin only after a one-hour lag. Second, callose synthesis, at least in oat protoplasts, requires protein synthesis: nieireatment with cycloheximide for at least three hours prior to victorin completely blocks callose production (Schaeffer and Walton, unpublished results). Finally, we have found that in vitro callose synthase activity is elevated in membrane preparations from victorin-treated protoplasts, when assayed with optimal concentrations of cellobinse, calcium, and UDPG. This last result is inconsistent with increased callose biosynthesis in vivo being due to a reversibly-bound metallic co-factor. 
However, the increased in vitro activity is only up to four-fold, whereas callose production in vivo increases at least ten-fold in response to aluminum or victorin (Walton and Earle, 1985; Schaeffer and Walton, 1990).

\section{References}

Anderson, A.J. Phytopathology 68:1585 (1978).

Cervone, F., M.G. Hahn, G. De Lorenzo, A. Darvill and P. Albersheim. Plant Physiol. 90:542 (1989).

Collins, R.A. and B.J. Saville. Nature 345:177 (1990).

Collmer, A. and N.T. Keen. Annu. Rev. Phytopathol. 24:383 (1986).

Heim, D., R.O. Nicholson, S.F. Pascholati, A.E. Hagerman, and W. Billet. Phytopathology 73:424 (1983).

Kauss, H. Annu. Rev. Plant Physiol. 38:47 (1987).

Kim, S.-D., H.W. Knoche and L.D. Dunkle. Physiol. Mol. Plant Pathol. 30:433 (1987).

Kleinkauf, $H$. and $H$. von Döhren. In: Blochemistry of Peptide Antibiotics $(H$. Kleinkauf and $H$. von Döhren, eds.), pp. 1-31, Walter De Gruyter, Berlin (1990).

Leonard, K.J. Phytopathology 63:112 (1973).

Lewin, R. Science 217:42 (1982).

Luckner, M. Secondary Metabolism in Microorganisms, Plants, and Animals, Springer-Verlag, Berlin (1984).

Mayama, S., T. Tani, T. Ueno, S.L. Midland, U.J. Sims and N.T. Keen. Physiol. Mol. Plant Pathol. $29: 1$ (1986).

Schaeffer, H.J. and J.D. Walton. Plant Physiol. 94:13 (1990).

Scott-Craig, J.S., D.G. Panaccione, F. Cervone, and J.D. Walton. The Plant Cell 2:1191 (1990).

Walton, J.D. Proc. Natl. Acad. Scl. USA 84i:8444 (1987).

Walton, J.D. In: Biochemistry of Peptide Antibiotics (H. Keinkauf and H. von Döhren, eds.), pp. 179-203, Walter de Gruyter, Berlin (1990).

Walton, J.D. In: Molecular Industrial Mycology (R. Berka and S. Leong, eds.), pp. 225-249, M. Dekker, New York (1991).

Walton, J.D. and F. Cervone. Physiol. Mol. Plant Pathol. 36:351 (1990).

Walton, J.D. and E.D. Earle. Planta 165:407 (1985).

Walton, J.D. and F.R. Holden. Mol. Plant-Microbe Interact. 1:128 (1988).

Wolpert, T.J., V. Macko, W. Acklin, B. Jaun, J. Seibl, J. Meili and D. Arigoni. Experientia 41:1524 (1985). 


\title{
DEVELOPMENTAL BIOLOGY OF NITROGEN-FIXING CYANOBACTERIA
}

\author{
C. Peter Wolk. Todd Biack, Yuping Cai, Jeff Elhai, Anneliese Ernst, Doron \\ Holland, Tanya Kuritz, Alicia Muro-Pastor, Elaine Oren, Kelly Owens and \\ Jean-Michel Panoff
}

Certain cyanobacteria (blue-green algae) are the only free-living prokaryotes capable of both oxygenic (i.e., higher-plant type) photosynthesis and reduction of nitrogen gas $\left(\mathrm{N}_{2}\right)$ to ammonia with light as the sole source of energy and water as the source of reductant. Despite the oxygen $\left(\mathrm{O}_{2}\right)$-lability of the $\mathrm{N}_{2}$-fixing enzyme system, these microbes are able to fix $N_{2}$ while producing $\mathrm{O}_{2}$ by photosynthesis. Nitrogen fixation in almost all of these prokaryotes is closely associated with cellular differentiation and intercellular interactions. Vegetative cells, which photosynthesize, grow and divide, can differentiate into two other types of cells: heterocysts and spores. Heterocysts are the sites of $\mathrm{N}_{2}$ fixation. However, they depend for this activity upon products of photosynthesis that they receive from the vegetative cells, and in return they supply products of $\mathrm{N}_{2}$ fixation to the vegetative cells. Spores are resistant cells that permit the organisms to survive periods of environmental stress.

We have previously shown that intercellular chemical interactions take place in filamentous cyanobacteria, and that heterocysts control both the differentiation of vegetative cells into additional heterocysts and the juxtaposition of spores and heterocysts. Our objectives based on this work are to define the intercellular interactions and biochemical processes that control the localization and dynamics of cellular differentiation and, once differentiation has taken place, that underlie nitrogen fixation. Having developed techniques for genetic analysis of these organisms, we are making extensive use of genetics to approach our objectives.

\section{Use of transposons, and luciferase as a promoter probe, to identify environmentally responsive genes in the heterocyst-forming cyano- bacterium, Anabaena sp. strain PCC 7120}

When deprived of fixed nitrogen, Anabaena initiates the differentiation of $\mathrm{N}_{2}$-fixing cells called heterocysts at semiregular intervals along its filaments. Models of the mechanism of this pattern formation depend on the nature of the spatial relationship, if any, between the cells that differentiate and the cells that first sense N-deprivation (Wolk, 1989). We therefore wish to identify the latter cells. Because it is possible to localize transcription of specific genes to individual cells of a living filament (Elhai and Wolk, 1990), we sought to identify Anabaena genes that are activated rapidly in response to removal of fixed nitrogen.

Genes that are activated upon a change in environmental conditions can be mutagenized under conditions in which the genes are inessential, and then 
identified by their response to alteration of those conditions. Seeking to identify such genes, we have used as a mutagen Tn5-1063, a derivative of Tn5 that incorporates the Vibrio fischeri lUXAB genes as a transcriptional reporter and confers resistance to neomycin $(\mathrm{Nm})$, bleomycin $(\mathrm{Bm})$ and streptomycin $(\mathrm{Sm})$. Tn5 transposes in Arabaena (Borthakur and Haselkorn, 1989). We monitiored the luminescence of libraries of transposition-derived colonies by use of a Hamamatsu Photonic System model C1966-20 (Photonic Microscopy, Inc., Oak Brook, IL) coupled to a Nikon $35-\mathrm{mm}$ i 2.0 macrotens. Several $\mu$ l of n-decanal, spread inside the top of a Petri dish, provided substrate for luciferase. Sources of differential luminescence Identifled by comparing images of luminescence made under different environmental conditions were localized to specific colonies by further comparison with corresponding analog (bright-field) Images.

Mutants responding rapidly to $\mathrm{N}$-deprivation by increase or decrease of luminescence were identified upon transfer of mating filters bearing transpositionderived colonies from agar plates containing nitrate to ones lacking fixed $\mathrm{N}$. Two purified mutants, TLN2 and TLN6, showed increased luminescence within 1 or 4 $\mathrm{hr}$, respectively. Luminescence increased equivalently if $\mathrm{NaNO}_{3}$ and $\mathrm{KNO}_{3}$ were omitted or replaced with equimolar $\mathrm{NaCl}$ and $\mathrm{KCl}$. Because TLN2 responds rapidly, it may react to $\mathrm{NO}_{3}^{-}$concentration per se, rather than to a $\mathrm{NO}_{3}{ }^{-}$-derived metabolic pool. Southern blots of total DNA from either mutant, restricted with EcoRI, EcoRV, and Clal, and probed with plasmid fragments containing transposon Tn5-1063a, showed only a single band of hybridizing DNA, implying that the genome bears only one copy of the transposon. Sequences of DNA contiguous with the transposon were recovered by digestion of total DNA with Clal or ECQRV, self-ligation (the transposon contains an origin of replication functional in $E$. colli), and transfer to $E$. coli by electroporation.

Restriction with Sall and hybridization with DNA from luXAB localized the transposon in TLN2 and TLN6 within fragments SalB and SalJ on the map of Bancroft et al. (1989), ca. 170 and $80 \mathrm{~kb}$ from the nearest Sall sites, respectiveiy. Hybridization with DNA from IS50R after restriction with a combination of AvrII plus Sphl localized the transposon in TLN2 to a position of $3.5 \mathrm{Mb}$ on that map, with luXAB pointing clockwise. TLN6 was tentatively localized at $4.2 \mathrm{Mb}$, with I $U \times A B$ criented counterclockwise.

Strains TLN2 and TLN6 differentiate normally. We are trying to identify, by transposon mutagenesis, regulatory genes whose products control the activation of the genes mutated in those strains. Such regulatory genes may also control differentiation. Our work thus represents a first step toward determining whether heterocyst differentlation is "the culmination of a process which begins as a nondevelopmental response to nitrogen deprivation" (Wolk, 1982), or whether that differentiation is elicited independently, for examble by more stringent $\mathrm{N}$ deprivation. 
We have isolated, similarly, mutants in which transcription of luxAB is activated by transfer from phosphate-sufficient to phosphate-deficient conditions, and mutants (including TLT2) in which luminescence is much (ca. 100-fold) greater after $24 \mathrm{hr}$ at $20^{\circ} \mathrm{C}$ than after incubation at $30^{\circ}$. Mutant TLT2 diminished in luminescent intensity with $t_{1 / 2} \approx 2.6 \mathrm{hr}$ when returned to $30^{\circ}$. Because desaturases of fatty acids become more active at lower temperatures, perhaps as a result of transcriptional activation, we analyzed the fatty acid composition of the lipids of these mutants following growth at $20^{\circ}$. No gross change in the desaturation of their fatty acids was observed, compared with other transpositionderived colonies and with wild-type Anabaena (S. Gibson, J.-M. Panoff, C.P. Wolk and $\mathrm{C}$. Somerville, unpublished data). As with proteins that are induced in Escherichia coli by cold shock (VanBogelen and Neidhardt, 1990), expression of TLT2 can be induced at $30^{\circ}$ by certain antibiotics that target the prokaryotic ribosome, namely chloramphenicol $(\mathrm{Cm})$, erythromycin $(\mathrm{Em})$, spectinomycin, spiramycin and tetracycline. (Supported in part by NSF grant DCB-8702368.)

\section{Regulation and localization of expression of hetA}

Using a sacB-containing vector, the hetA gene (Wolk et al., 1988) was replaced by a transcriptional fusion of the hetA promoter with luxAB. The resulting strain was subjected to transposon mutagenesis with Tn5-1058, the lux-less progenitor of Tn5-1063. Whereas luminescence of the parent strain was induced by nitrogen-deprivation, mutagenized derivatives were identified in which luminescence was not thereby induced, and others in which luminescence was expressed constitutively in the presence of nitrate. A majority of the mutants of the latter group were found to be deficient in uptake or utilization of nitrate. However, three mutants that expressed hetA in the presence of nitrate did not form heterocysts in the presence of nitrate but did in the absence of nitrate. One of these mutants had a transposon insertion between the hetA promoter and the luciferase reporter; the transposon in the remaining two mutants was not near the hetA gene. The implication of these observations is that at least one gene (hetA) involved in stability of the heterocyst envelope may be subject to both positive and negative regulation.

Whereas $P_{\text {rbc }}$ luxAB and $P_{\text {nif }}$ luXAB show strong luminescence at the single cell level (Elhai and Wolk, 1990), single-cell luminescence from $P_{\text {hetA }}$ luXAB is feeble. The signal, amplified 15 -fold by use of a combination of (i) a fusion ( $P_{\text {hetA }}$ rpoT7) of the promoter of hetA to the structural gene for T7 RNA polymerase and (ii) plasmid pRL1050, bearing a fusion of a promoter recognized by $T 7$ polymerase, to $\underline{\mathrm{l} X \mathrm{AB}}$, appeared to localize luminescence to proheterocysts. In order to test whether the localization of luminesence seen from the Anabaena derivative carrying $P_{\text {hetA }}$ rpoT7 is due to inactivity of T7 polymerase in vegetative cells, eight derivatives of Anabaena PCC7120 [PRL1050] carrying independent insertions of Tn5-1065 (with rpoT7 as reporter gene) were selected for their ability to express luciferase well when grown on nitrate. They exhibited a range of luminosity in 
liquid, ranging from 1.2 to $13 \times 10^{10}$ quanta/min $\mu \mathrm{g}$ chlorophyll. Three strains, chosen for more detalled study, were shifted to a nitrate-free medium. After 11 $h$ of starvation for nitrogen, filaments were examined under the microscope. In all three cases, luminescence was visible from vegetative cellis. T7 polymerase evidently is able to couple promoter activity to lucliferase expression in both developing and nondeveloping cells. Luminescence amplified by means of the T7 polymerase-luclferase couple appears then to reflect, at least qualitatively, the spatial ac tivity of regulated promoters and may be used to detect the sites of weak, regulated transcription. (Supported in part by NSF grant DCB-8702368.)

\section{Screening for mutants incapable of aerobic fixation of $\mathbf{N}_{2}$}

Ca. 1 to $2 \%$ of transposon-mutated colonies, unable to flx $\mathrm{N}_{2}$ aerobically, become and remain yellow after a filter bearing small colonies is transferred from $+\mathrm{N}$ to $-\mathrm{N}$ conditions. Such mutants were purified, and examined by microscopy 7 days after transfer to $A A$ medium containing $10 \mu \mathrm{g} \mathrm{Sm} / \mathrm{ml}$. All but a small proportion of the mutants make heterocysts, although the heterocysts in many mutants are morphologically aberrant. Notable mutants include two which lack, apparently specifically, the envelope glycolipids of heterocysts, and eight (including $\alpha-41$ and $\alpha-63$; see below) which lack obvious differentiation. Three of the eight transpositions are close together in the chromosome, while four others are in unique locl. The indicated phenotypes have been observed previously (Haury and Wolk, 1978; Spence and Stewart, 1987; Wilcox et al., 1988; Wolk et al., 1988; Buikema and Haselkorn, Abst. Molec. Biol. of Cyanobacteria Workshop, Toronto, 1989).

\section{Insertion sequences}

We earlier showed (Cai and Wolk, 1990) the presence in Anabaena sp. str. PCC 7120 of at least 6 distinct insertion sequences, so that there is always the possibility that upon selecting for a transposon, we obtain resistance due to the transposon but a phenotype due to transposition of an insertion sequence. The transposon and sequences contiguous to it should, therefore, be transferred to a new strain to test whether the phenotype follows it. Vector PRL1075, which contains SacB and oriT (RK2), and confers resistance to Cm and Em, has been developed for this purpose and was used to show that the phenotypes of $\alpha-41$ and $\alpha-63$ are indeed due to the transposon. Other vectors have been and are being constructed to facilitate replacement of transposons by reporter genes, antecedent to further transposon mutagenesis.

This year also saw the sequencing of IS892, one of a family of at least 9 related insertion sequences in PCC 7120, not all of which are identical. IS892 is 1675 base pairs in size with 24-bp near-perfect inverted terminal repeats, generates an 8-bp directly repeated target duplication upon insertion into its target site, and has two open reading frames (ORFs) that code for proteins of 233 and 137 amino 
acids, respectively. The inverted terminal repeats of this IS element make it more similar to "typical" insertion sequences isolated from other bacteria than to IS891, an IS element isolated from another strain of Anabaena, Anabaena M-131.

It has been suggested that the $E$. coli integration host factor (IHF), a histone-like DNA binding protein, plays a strong stimulatory role in many types of site-specific recombination, including transposition of transposable elements. Along with the primary enzyme that catalyses the site-recombination reaction, the IHF protein is thought to participate in wrapping the recombination site into a nucleosomelike structure. An extended 48 bp-long consensus sequence of the binding site for IHF has been proposed based on a compllation of published studies. Computer search revealed two possible IHF-binding sites in the sequence of IS892 that closely match the consensus sequence. These two sites are both on the coding strand and are located ca. 140 bp from either end of IS892. Anabaena is evolutionarily distant from $E$. coli by various means of measurement. The presence of two possible IHF-binding sites in IS892 suggests that an IHF-like mechanism of recombination may be widespread among prokaryotes.

\section{Further efforts to facilitate gene transfer to strains of Anabaena}

Plasmid pRL528, which methylates Aval and Sau96l (including Avall) sites, has proven very helpful for research with Anabaena sp. PCC 7120 (Golden \& Wiest, 1988; Borthakur \& Haselkorn, 1989; Elhai \& Wolk, 1990), which would otherwise restrict conjugally transferred DNA bearing those sites. Our preliminary evidence indicates that the presence of unmethylated Avalli sites also leads to restriction. Plasmid pEC22 (Yoshida and Mise, 1986), which contains genes for restriction and methylation of sites for EcoT22I, an isoschizomer of Avalll, was therefore mapped, and the methylase gene subcloned as a 1.2-kb Hpall fragment, in preparation for its insertion into pRL528.

Extracts of Anabaena variabilis ATCC 29413, a heterotrophic strain, have very high activity of a non-specific nuclease that degrades RNA and single- or doublestranded DNA extensively. Much weaker activity of a corresponding nuclease is observed in extracts of Anabaena sp. PCC 7120. In collaboration with A. MuroPastor and E. Flores of U. Sevilla, Spain, we have cloned the gene for the nuclease from Anabaena 7120, and used it to inactivate, site-specifically, the corresponding nucleases from the two strains of Anabaena. It will be very interesting to determine whether, as is true of a somewhat similar nuciease from Streptomyces glaucescens (Aparicio et al., 1988), this nuclease serves a restriction function in Anabaena 29413, and whether its loss will permit the isolation of DNA large enough to permit pulse-field gel mapping of the chromosome of the latter strain and/or facilitate the isolation of such DNA from Anabaena PCC 7120. 


\section{References}

Apariclo, J.F., C.G. de los Reyes-Gavilán, C. Barbes, C. Hardisson and J. Sanchez. J. Gen. Microbiol. 134:2345 (1988).

Bancroft, I., C.P. Wolk and E.V. Oren. J. Bacteriol. 171:5940 (1989).

Borthakur, D. and R. Haselkorn. J. Bacteriol. 171:5759 (1989).

Cai, Y. and C.P. Wolk. J. Bacteriol. 172:3138 (1990).

Currier, T.C., J.F. Haury and C.P. Wolk. J. Bacteriol. 129:1556 (1977).

Elhal, J. and C.P. Wolk. EMBO J. 9:3379 (1990).

Golden, J.W. and D.R. Wiest. Science 242:1421 (1988).

Haury, J.F. and C.P. Wolk. J. Bacteriol. 136:688 (1978).

Spence, D.W. and W.D.P. Stewart. FEMS Microbiol. Lett. 40:119 (1987).

VanBogelen, R.A. and F.C. Neidhardt. Proc. Natl. Acad. Scl. USA 87:5589 (1990).

Wilcox, M., G.J. Mitchison and R.J. Smith. Arch. Microbiol. 103:219 (1975).

Wolk, C.P. In: The Biology of Cyanobacteria (N.G. Carr, B.A. Whitton, eds.)

Blackwell Scient. Publ., Oxford, U.K., pp. 359-386 (1982).

Wolk, C.P. Plant Syst. Evol. 164:27 (1989).

Wolk, C.P., Y. Cai, L. Cardemil, E. Flores, B. Hohn, M. Murry, G. Schmetterer, B.

Schrautemeier and R. Wilson. J. Bacteriol. 170:1239 (1988).

Yoshida, Y. and K. Mise. J. Bacteriol. 165:357 (1986). 


\title{
ENVIRONMENTAL CONTROL OF PLANT DEVELOPMENT AND ITS RELATION TO PLANT HORMONES
}

\author{
Jan Zeevaart. Robert Bensen, Fancoise Fantauzzo, Christopher Rock, Manuel \\ Talon and Theresa Wilson
}

Environmental factors such as photoperiod, light quality, temperature, and water deficits have pronounced effects cn plant growth and development. Plant growth substances are often involved as intermediaries between the perception of an environmental factor and the ultimate morphological expiession. The aim of this task is to elucidate how environmental factors regulate the metabolism and action of these plant growth substances. In this context, the following topics are under investigation: a) Photoperiodic control of stem growth in long-day rosette plants by gibberellins. The objective is to elucidate how light regulates gibberellin biosynthesis in these plants, how changes in gibberellin status are brought about at the biochemical and molecular level, and how these changes result in stem growth. These studies on gibberellins in rosette plants, the so-called "physiological dwarfs", are complemented by characterization of a series of singlegene dwarf mutants ("genetic dwarfs") in tomato and Arabidopsis which are deficient in gibberellins. The immediate objective is to determine which steps in the gibberellin biosynthetic pathway are blocked in these mutants, and further to use these mutants for molecular genetic studies. As a whole, this research will lead to a better understanding of the environmental regulation of plant growth and productivity. b) Biosynthesis of abscisic acid and its catabolites induced in response to water stress. The aim is to work out the biosynthetic pathway of abscisic acid, to determine the rate-limiting step that is stimulated by water stress, and to elucidate how a large increase in abscisic acid alleviates the effects of stress. The results of these studies will lead to a better understanding of how plants are able to cope with stress.

\section{Studies on Gibberellins}

\section{Only 3ß-hydroxylated gibberellins are active per se in stem growth of spinach}

Spinach is a long-day (LD) rosette plant in which stem elongation under LD conditions ( $8 \mathrm{~h}$ high-intensity light, $16 \mathrm{~h}$ low-intensity light) is mediated by GAs. In previous work (Plant Research '89, pp. 117-118) it has been shown that the early-13-hydroxylation pathway $\left(G A_{53}, \mathrm{CA}_{44}, \mathrm{GA}_{19}, \mathrm{GA}_{20}, \mathrm{GA}_{1}, \mathrm{GA} \mathrm{A}_{8}\right)$ is the major biosynthetic pathway in spinach. The 'sB-hydroxylated $G A s, G_{4}$ and $G A_{7}$, have also been identified in spinach, but their levels are always very low. In the experiments reported here the growth retardants AMO-1618 (which inhibits kaurene synthetase $A$ activity) and prohexadione or BX-112 (which inhibits dioxygenases that require 2-oxoglutarate as co-substrate) (Nakayama et al., 1990) were used to determine which GA is active per se in spinach. As shown in Table 1, both $A M O-1618$ and $B X-112$ suppressed stem growth in plants under $L D$. In the case of $A M O-1618$, applied $G A_{20}$ and $G A_{1}$ were equally effective in overcoming the 
growth Inhibition, but with $\mathrm{BX}-112$ only $\mathrm{GA}_{1}$, not $\mathrm{GA}_{20}$, could restore normal growth. $\mathrm{GA}_{4}$ applied to plants treated with $\mathrm{BX}-112$ was just as effective as $G A_{1}$ in causing stem growth. Analyses of endogenous $G A s$ in plants treated with $B X-112$ showed that the levels of $\mathrm{GA}_{1}$ and $\mathrm{GA}_{8}$ were much reduced, while $\mathrm{GA}_{20}$ and $\mathrm{GA}_{19}$ accumulated. These findings indicate that the main effect of $B X-112$ in spinach is inhibition of the 3B-hydroxylation step from $\mathrm{GA}_{20}$ to $\mathrm{GA}_{1}$. It also establishes unequivocally that $3 B$-hydroxylation is required for $G A$ activity in spinach. (Supported, in part, by a fellowship from the Spanish Ministry of Agriculture to Manuel Talon, and by USDA grant \#88-37261-3434.)

Table 1. Effects of the growth retardants $A M O-1618$ and $B X-112$ and of the gibberellins, $\mathrm{GA}_{1}$ and $\mathrm{GA}_{20}$, on stem length $(\mathrm{cm})$ in spinach. Measurements after 20 LD

\begin{tabular}{lccc} 
& \multicolumn{3}{c}{ Growth retardant } \\
GA & None & AMO-1618 & BX-112 \\
\hline None & 30.4 & 1.4 & 1.0 \\
GA $_{20}$ & $\cdots$ & 33.8 & 1.9 \\
$\mathrm{GA}_{1}$ & & 31.1 & 31.2
\end{tabular}

Fluctuations in gibberellin levels under various light-dark conditions

With the use of deuterated GAs as internal standards, accurate and reproducible determinations of GAs can be made in relatively small tissue samples. Such measurements have been carried out with plants grown under SD conditions, or after transfer from SD to LD. In SD, the GA levels fluctuated, increasing during the light, and decreasing during the dark period. After transfer from SD to $L D, G_{53}$ and $\mathrm{GA}_{19}$ decreased significantly during the first supplementary light period, indicating continued activity of $\mathrm{GA}_{53}$ - and $\mathrm{GA}_{19}$-oxidases, whereas $\mathrm{GA}_{20}$ increased. During the following high-intensity light period the $\mathrm{GA}_{20}$ level decreased and $\mathrm{GA}_{1}$ increased. These fluctuations in $G A$ levels continued in $L D$, resulting in an increase in absolute levels of $\mathrm{GA}_{20}$ and $\mathrm{GA}_{1}$ which in turn resuited in stem growth. These observations are compatible with the idea that the flow through the GA biosynthetic pathway is much enhanced in LD, particularly during the period of high-intensity light, as compared to SD and darkness. (Supported, in part, by a fellowship from the Spanish Ministry of Agriculture to Manuel Talon, and by USDA grant \#88-37261-3434.) 
Cellular changes induced by exogenous and endogenous gibberellins in the shoot tips of Silene armeria

Previous studies suggested that in the long-day rosette plant, Silene armeria, strain $S 1.2$, accumulation of $\mathrm{GA}_{1}$ in the shoot tips of LD-grown plants causes stem growth (Talon and Zeevaart, 1990). One of the early effects of LD induction or of GA application in SD is an increase in mitotic activity in the subapical meristem (Sachs, 1961).

In the present investigation on Silene, microscopic observations on early changes at the cellular level following LD induction or GA treatment in SD, were combined with GA analyses of sections located at various distances below the shoot apex. Within 8 days after the beginning of LD induction or after GA application to plants in SD, the cells in the subapical meristem were of similar size and were predominantly oriented in long files (Fig. 1B, C), indicating that many transverse cell divisions had taken place. In contrast, in shoot tips of plants in SD the cells in the subapical region varied in size and were not oriented in any particular direcuon (Fig. 1A). Staining longitudinal sections of induced plants with calcofluor white showed that cellulose deposition occurred mostly in the longitudinal walls, whereas in the control plants in SD both longitudinal and transverse walls were stained. This indicates that in induced plants the cells elongated mainly in the longitudinal direction, giving rise to an elongated stem.

A

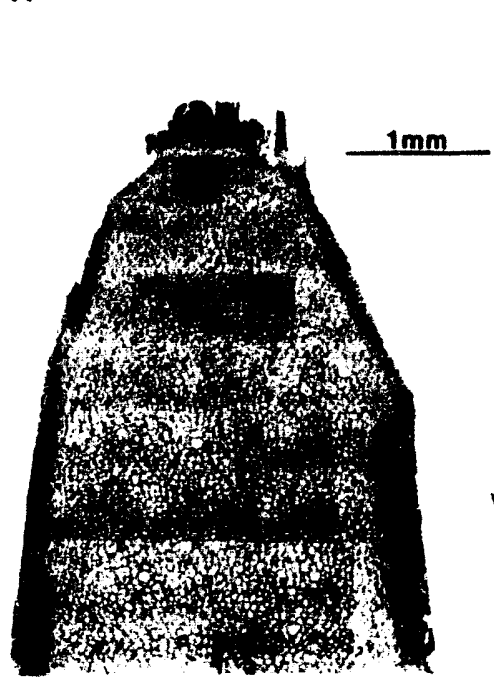

B

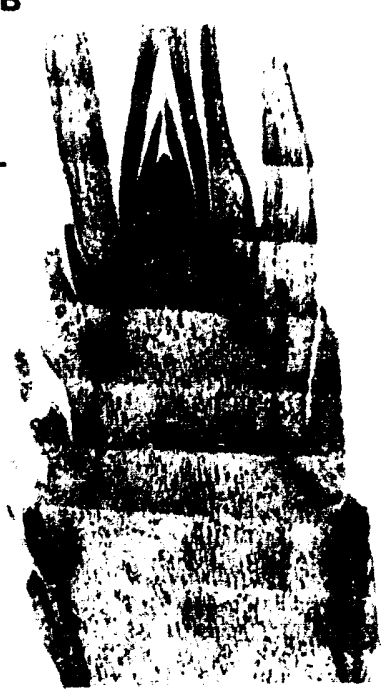

C

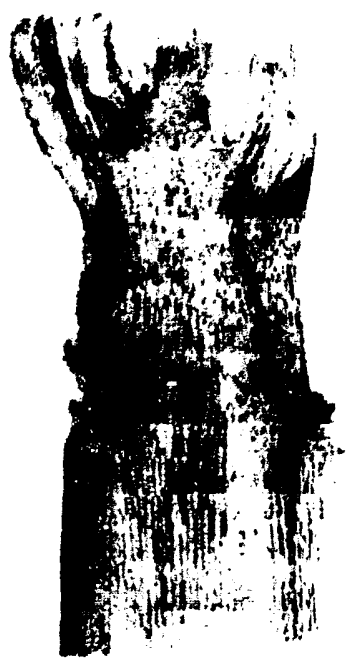

Fig. 1. Longitudinal sections through shoot apices of Silene armeria. A: From plants in SD; B: From plants after 8 LD; C: From plants in SD treated with GA;. 
Material for GA analyses was obtained by cutting thin sections at various distances below the shoot apex. Endogenous GAs increased in all sections of the subapical meristem from plants in $L D$ as compared to those from SD. For example, in the zone $500-1500 \mu \mathrm{m}$ below the apex, the GA content increased from $4 \mathrm{ng} / \mathrm{g}$ dry wt. in sections from SD to $136 \mathrm{ng} / \mathrm{g}$ dry wt. in material harvested after $8 \mathrm{LD}$. Thus, an increase in GA content either by application in SD or following LD induction stimulated cell division in the subapical meristem which resulted in stem elongation. LD treatment not only induced stem elongation, but also floral initiation, whereas plants in SD treated with GA produced stems, but no flower primordia (Fig. 1C). Thus, although applied GA mimics the effects of LD on stem growth, it does not induce floral initiation in this strain of Silene Wellensiek, 1973). These observations illustrate, therefore, that stem elongation in rosette plants is controlled by GAs, whereas floral initiation is also induced by LD, but via a different mechanism (Zeevaart, 1983). (Work done in cooperation with Dr. F.R. Tadeo, INRA, C. R. Bordeaux, Station de Recherches d'Arbiculture Fruitières, 33883 Villenave d'Ornon Cedex, France; supported, in part, by a fellowship from the Spanish Ministry of Agriculture to Manuel Talon, and by USDA grant \#88-37261-3434.)

\section{Characterization of GA-responsive, dwarf mutants of Arabidopsis thaliana}

There are five non-allelic dwarf mutants in Arabidopsis which are GA biosynthetic mutants (Koornneef and van der Veen, 1980). Experiments directed towards determining the biosynthetic step at which each of these mutants is blocked have been performed. By analysis of the endogenous GAs and quantitation of their levels the ga4 and gas mutants were found to be blocked late in the GA biosyntinetic pathway. A similar analysis of endogenous GAs in the ga1, ga2, and ga3 mutants indicated that all three are blocked at steps prior to $\mathrm{GA}_{12}$ syrithesis (Talon and Zeevaart, unpublished). Furthermore, application of ent-kaurene and all precursors later in the GA pathway cause a growth response in ga1 and ga2 plants, whereas ga3 plants respond to applied ent-kaurenoic acid and all later GA precursors. These results suggest that the ga1 and ga2 mutants are blocked at steps prior to ent-kaurene, presumably at kaurene synthetase $A$ and $B$, while ga3 is blocked in the oxidation of ent-kaurenal to ent-kaurenoic acid (Plant Research '86, pp. 130-131).

For further characterization of these mutants, the ability to convert geranylgeranyl pyrophosphate (GGPP) to copalyl pyrophosphate (CPP) and CPP to ent-kaurene, was measured in cell-free extracts of immature siliques from normal and mutant plants, as was done previously with GA-deficient mutants of tomato (Bensen and Zeevaart, 1990). Based on tentative identifications of the products CPP and entkaurene by HPLC, it was found that the ga3 mutant was deficient in kaurene synthetase $A$ activity, whereas the ga2 mutant lacked $B$ activity. In the case of the ga1 mutant, both $A$ and $B$ activities were comparable to those measured in extracts from normal plants. 
It is clear that the observations made with applications of precursors to mutant plants and measurements of kaurene synthetase A and B activities in extracts from immature siliques are not in agreement with respect to the blocks in the ga1 and gaß mutants. Additional experiments are required to precisely locate the blosynthetic lesions in these mutants.

\section{Molecular analysis of the ga4 and 985 mutants of Arabidopsis thaliana}

The GA4 and GA5 locl of Arabidopsis code for genes involved in GA biosynthesis, with mutations in these genes resulting in semi-dwarf plant stature. The ga4 mutant is partially blocked in the $3 B$-hydroxylation step $\left(G A_{20}\right.$ to $G A_{1}$, and $G A_{6}$ to $\mathrm{GA}_{4}$ ), while the gas mutant is partially blocked in $\mathrm{C}-20$ hydroxylation ( $\mathrm{GA}_{3}$ to $\mathrm{GA}_{44}$ ) and $\mathrm{C}-20$ elimination $\left(\mathrm{GA}_{19}\right.$ to $\mathrm{GA}_{20}$ ) (Talon et al., 1990). Both lesions occur in steps that are of interest for studying the regulation of GA biosynthesis. The 3Bhydroxylation step is the penultimate step in the synthesis of bioactive GA, whereas the conversions of $\mathrm{GA}_{53}$ to $\mathrm{GA}_{44}$, and $\mathrm{GA}_{19}$ to $\mathrm{GA}_{20}$ in spinach are known to be regulated by light (Gilmour et al., 1986; Zeevaart et al., 1990). Thus, the molecular aspects of the regulation of these activities may yield important clues as to how GAs affect stem elongation rates in response to light.

In order to study the regulation of these genes, we have initiated a project aimed at obtaining genomic clones of the GA4 and GA5 loci. Our aim is to clone these loci by chromosome walking using an Arabidopsis genomic library cloned into Yeast Artificial Chromosomes (YACs). This involves mapping the GA4 and GA5 loci relative to RFLP markers, selection of YACs containing Arabidopsis DNA sequences that are homologous to proximal-flanking RFLPS, PCR assisted walking in the YAC library, and complementation of the lesion by Agrobacterium mediated transformation of each mutant.

\section{Studies on Abscisic Acid}

Further studies on abscisic acid biosynthesis in the flacca and sitiens mutants of tomato

We have reported ${ }^{18}$ Olabeling experiments which indicated that the ABA-deficient mutants flacca and sitiens of tomato synthesize a large proportion of $A B A$ and trans-ABA from $A B A$-alcohol and trans-ABA-alcohol, respectively (Plant Research ' 89, p. 119). We have further investigated the shunt pathway from $A B A$-aldehyde $\rightarrow$ ABA-alcohot $A B A$ by feeding $\left.{ }^{2} \mathrm{H}_{5}\right] \mathrm{ABA}$-alcohol to wild type and flacca mutant leaves in an atmosphere with $20 \%{ }^{18} \mathrm{O}_{2}$. Both wild type and mutant tissues converted $\left.{ }^{2} \mathrm{H}_{5}\right] \mathrm{ABA}$-alcohol to ${ }^{2} \mathrm{H}_{6}^{18}$ O]ABA with similar efficiency which indicates that the shunt pathway is not exploited by the mutants to maximize $A B A$ synthesis. The conversion of [ $\left.{ }^{2} H_{5}\right] A B A$-alcohol to $\left.{ }^{2} H_{5}\right] A B A$ in sitiens leaves in the presence of carbon monoxide was inhibited $80 \%$, whereas in a nitrogen atmosphere this conversion was completely inhibited. These findings provide 
evidence for the involvement of a P-450 type monooxygenase which catalyzes the conversion of $A B A$-alcohol to $A B A$ with the incorporation of molecular oxygen. (Supported, in part, by NSF grant \#DMB-8703847.)

\section{Characterization of the aba mutant of Arabidopsis thaliana}

Three alleles of the ABA locus of $A$. thaliana are deficient in ABA (Koornneef et al., 1982). We have used ${ }^{18} \mathrm{O}_{2}$ to label $A B A$ in water-stressed leaves of mutant and wild-type Arabidopsis. Analysis by selected ion monitoring and tandem mass spectrometry of $\left[{ }^{18}\right.$ O]ABA and its catabolites showed that the mutant alleles are impaired in $A B A$ biosynthesis and that they have an abnormally small precursor pool containing the ring oxygens of $A B A$, presumably oxygenated carotenoids (xanthophylls). Quantitation of the carotenoids from mutant and wild type leaves showed that leaves of the mutants are deficient in the epoxy-carotenoids, violaxanthin and neoxanthin, and accumulate the biosynthetic precursor zeaxanthin (Fig. 2). These results provide strong evidence that ABA is a
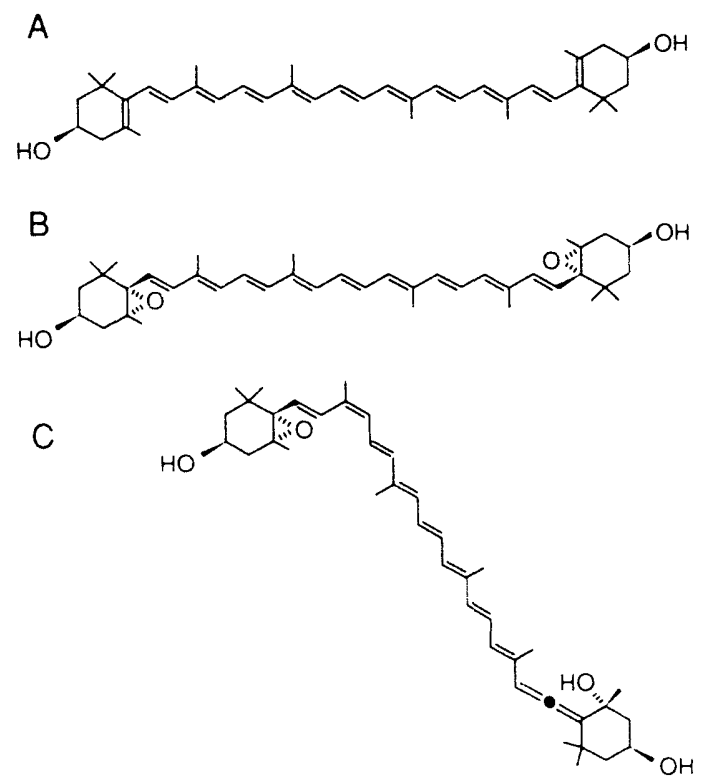

D

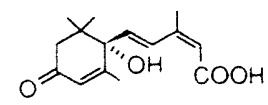

Figure 2. Structures of A)zeaxanthin; B) trans-violaxanthin; C) 9'-cis-neoxanthin; D) abscisic acid. 
breakdown product of xanthophylls. Interestingly, the different alleles of the aba mutant are less impaired in biosynt. lisis of trans-ABA than of ABA. Feeding studies with xanthoxin isomers suggest that trans-xanthoxin may be a precursor to $A B A$ in Arabidopsis. (Supported, in part, by NSF grant \#DMB-8703847.)

\section{Relationship between ${ }^{18} \mathrm{O}$ incorporation in carotenoids and abscisic acid}

In earlier labeling studies of ABA with ${ }^{18} \mathrm{O}_{2}$ it was established that in waterstresied leaves and unripe fruits ${ }^{18} \mathrm{O}$ enrichment is mainly in the carboxyl group, while only small amounts of ${ }^{18} \mathrm{O}$ are incorporated in the ring oxygens. However, in ripening fruits there is considerable ${ }^{18} \mathrm{O}$ enrichment of the oxygens on the ring (Zeevaart et al., 1989). These results suggest that xanthophylls, as presumed ABA precursors, must become ${ }^{18} \mathrm{O}$ labeled to a different extent in the various tissues analyzed. The present experiments were undertaken to determine whether the ${ }^{18} \mathrm{O}$-labeling pattern of $A B A$ is related to that of the xanthophylls, violaxanthin and neoxanthin.

In ABA from apple tissue, ${ }^{18} \mathrm{O}$ enrichment of the ring oxygens was higher than that of the carboxyl group. In the same tissue, approximately $50 \%$ of the violaxanthin and $25 \%$ of the neoxanthin molecules had incorporated four ${ }^{18} \mathrm{O}$ atoms (Table 2). Thus, the labeling pattern of the xanthophylls closely paralleled that of ABA. The observation that the percentage labeled violaxanthin was greater than that of neoxanthin (Table 2) is consistent with the former being the precursor of the latter.

Table 2. ${ }^{18} \mathrm{O}$ Incorporation into violaxanthin and neoxanthin in cortical tissue of 'Mutsu' apple fruits'

\begin{tabular}{|c|c|c|c|c|c|}
\hline \multirow[b]{2}{*}{$\mathrm{m} / \mathrm{z}$} & \multirow[b]{2}{*}{${ }^{18} \mathrm{O}$} & \multicolumn{2}{|c|}{ Violaxanthin } & \multicolumn{2}{|c|}{ Neoxanthin } \\
\hline & & $\underline{\text { cis }}$ & $\underline{\operatorname{trans}}$ & cis & trans \\
\hline 600 & 0 & 26 & 30 & 52 & 64 \\
\hline 602 & 1 & 5 & 3 & 6 & 3 \\
\hline 604 & 2 & 10 & 3 & 6 & 4 \\
\hline 606 & 3 & 9 & 9 & 9 & 6 \\
\hline 608 & 4 & 50 & 55 & 28 & 23 \\
\hline
\end{tabular}

In unripe avocado fruit most of the. $A B A$ was labeled with a single ${ }^{18} \mathrm{O}$ in the carboxyl group, while in the same cissue the xanthophylls had incorporated little 
${ }^{18} \mathrm{O}$. In ripe avocado fruit, ${ }^{18} \mathrm{O}$ incorporation into $\mathrm{ABA}$ was low, with 2 or 3 atoms of ${ }^{18} \mathrm{O}$ more abundant than singly labeled ones. This corresponded to a high incorporation of ${ }^{18} \mathrm{O}$ into the xanthophylls. $\mathrm{ABA}$ accumulated during ripening of avocado fruit, while the xanthophyll pool decreased by $90 \%$. In their entirety, these results establish a close correlation between ${ }^{18}$ O labeling of the oxygens on the ring of $A B A$ on the one hand and ${ }^{18} \mathrm{O}$ incorporation by violaxanthin and neoxanthin on the other. They provide, therefore, further evidence that these xanthophylls are precursors to ABA. Work done in cooperation with D.A. Gage, MSU/NIH Mass Spectrometry Facility; supported, in part, by NSF grant \#DMB8703847.)

\section{References}

Bensen, R.J. and J.A.D. Zeevaart. J. Plant Growth Regul. 9:237 (1990).

Gilmour, S.J., J.A.D. Zeevaart, L. Schwenen and J.E. Graebe. Plant Physiol. 82:190 (1986).

Koornneef, M., M.L. Jorna, D.L.C. Brinkhorst-van der Swan and C.M. Karssen. Theor. Appl. Genet. 61:385 (1982).

Koornneef, M. and J.H. van der Veen. Theor. Appl. Genet. 58:257 (1980).

Nakayama, I., Y. Kamiya, M. Kobayashi, H. Abe and A. Sakurai. Plant Cell Physiol. 31:1183 (1990).

Sachs, R.M. Adv. Chem. Ser. 28:49 (1961).

Talon, M., M. Koornneef and J.A.D. Zeevaart. Proc. Natl. Acad. Sci. USA 87:7983 (1990).

Talon, M. and J.A.D. Zeevaart. Plant Physiol. 92:1094 (1990).

Wellensiek, S.J. Netherl. J. Agric. Sci. 21:245 (1973).

Zeevaart, J.A.D. In: The Biochemistry and Physiology of Gibberellins, Vol. 2 (A. Crozier,ed.) Praeger, New York, pp. 333 (1983).

Zeevaart, J.A.D., T.J. Heath and D.A. Gage. Plant Physiol. 91:1594 (1989).

Zeevaart, J.A.D., M. Talon and T.M. Wilson. In: Gibberellin Symposium Tokyo 1989, (N. Takahashi, J. MacMillan and B.O. Phinney, eds.) Springer, Berlin, pp. 273 (1990). 


\section{PERSONNEL}

\section{Faculty, and Joint Appointment}

Frans de Bruijn, Assistant Professor of Microbiology and Public Health, Ph.D. (Hanvard University)

Deborah P. Delmer, Adjunct Professor of Research, Ph.D. (University of California, San Diego)

Pamela J. Green, Assistant Professor of Biochemistry, Ph.D. (State University of New York)

Andrew D. Hanson, Professor of Crop and Soil Sciences, Ph.D. (University of London, United Kingdom)

Hans Kende, Professor of Botany and Plant Pathology, Ph.D. (University of Zurich, Switzerland)

Derek T.A. Lamport, Professor of Biochemistry, Ph.D. (University of Cambridge, United Kingdom)

Anton Lang, Professor Emeritus of Botany and Plant Pathology, Ph.D. (University of Berlin, Germany)

Lee Mclntosh, Professor of Biochemistry, Ph.D. (University of Washington)

Thomas Newman, Assistant Professor of Research, Ph.D. (Purdue University)

Kenneth L. Poff, Professor of Botany and Plant Pathology, Ph.D. (West Virginia University)

Natasha Raikhel, Associate Professor of Botany and Plant Pathology, Ph.D. (Academy of Sciences, Leningrad, USSR)

Christopher R. Somerville, Professor of Botany and Plant Pathology, Ph.D. (University of Alberta, Canada)

Shauna C. Somerville, Assistant Professur of Botany and Plant Pathology, Ph.D. (University of Illinois)

Jonathan D. Walton, Assistant Professor of Botany and Plant Pathology, Ph.D. (Stanford University)

C. Peter Wolk, Director, Professor of Botany and Plant Pathology, Ph.D.

(Rockefeller Institute)

Jan A.D. Zeevaart, Professor of Botany and Plant Pathology, Ph.D. (Agricultural University of Wageningen, The Netherlands)

\section{Visiting Scientists}

Jossi Hirschberg, Department of Genetics, The Hebrew University, Jerusalem, Israel, until August 31, 1990

Radomir Konjevic, Institute of Botany, University of Belgrade, Yugoslavia, from August 1, 1990 until September 24, 1990

Taka-Aki Ono, Laboratory of Plant Physiology, Riken Institute of Physical \& Chemical Research, Wako, Saitama, Japan, until May 31, 1990 
Research Associates, Postdoctoral Fellows, and Specialists (Supervising Faculty Member)

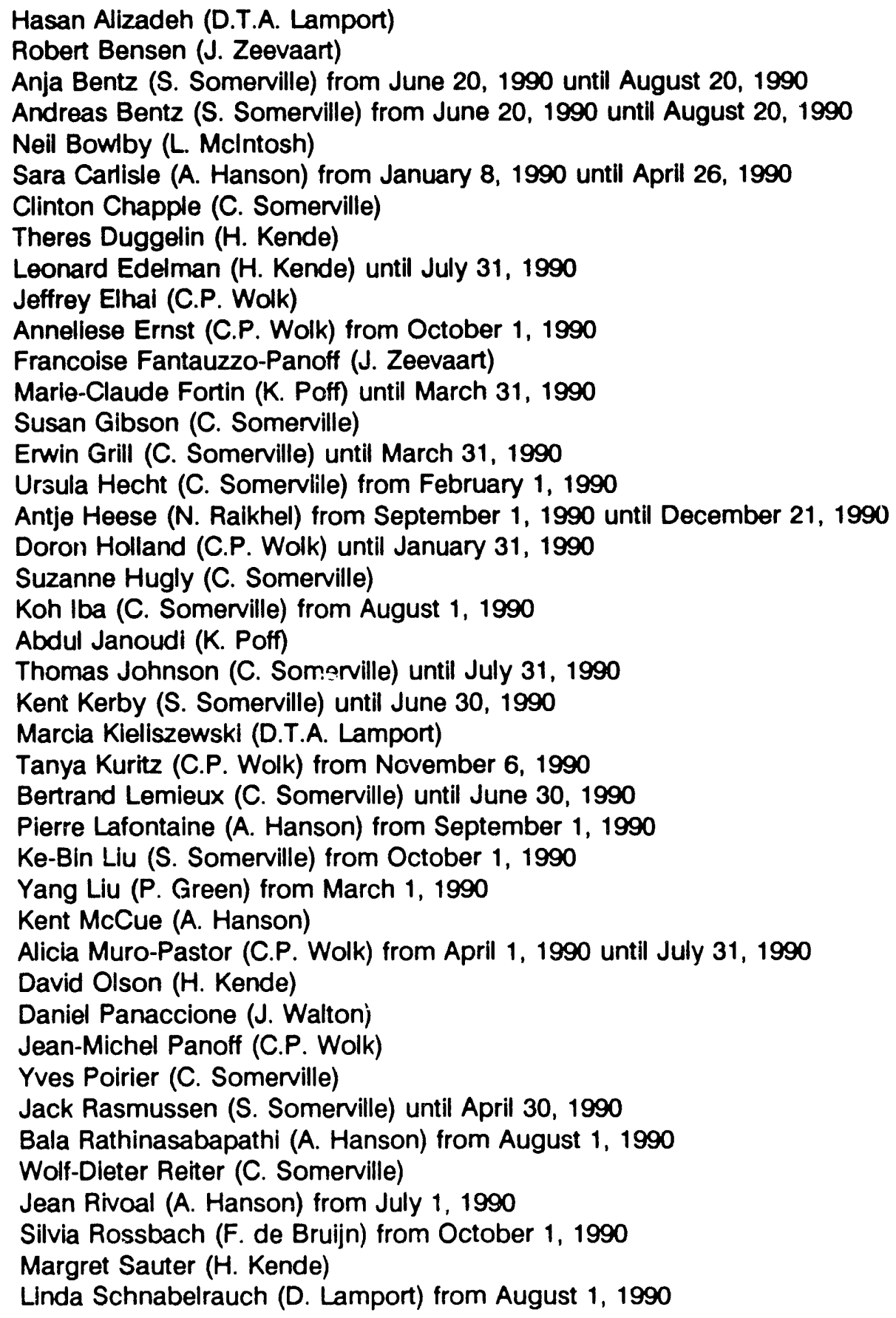


Jane Schneider (C. Somerville)

Martin Schroeder (N. Raikhel) from September 20, 1990

John Scott-Craig (J. Walton)

John Shanklin (C. Somerville)

Ida Sithole (L. Mclntosh)

Bernice Slutsky (S. Somerville) until March 26, 1990

Johannes Stigter ( $F$. de Bruijn) from July 1, 1990

Masaru Takagi (P. Green) from September 11, 1990

Manual Talon (J. Zeevaart)

Brad Upham (D. Lamport) from July 1, 1990

Marguerite Varagona (N. Raikhel)

Elizabeth Verkamp (P. Green) until February 1, 1990

Jennifer Weller (S. Somerville) from September 1, 1990

Joseph White ( $H$. Kende)

Theresa Wilson (J. Zeevaart)

Yang Yen (P. Green) from February 1, 1990

Graduate Assistants (Departmental affiliation and supervising faculty member)

Shawn Anderson (Botany and Plant Pathology) L. Mclntosh

Sebastian Bednarek (Botany and Plant Pathology) N. Raikhel

Lawrence Benbow (Botany and Plant Pathology) D.T.A. Lamport

Christoph Benning (Genetics) C. Somerville

Todd Black (Biochemistry) C.P. Wolk

Bertha Bullen (Botany and Plant Pathology) K. Poff

Yuping Cai (Botany and Plant Pathology) C.P. Wolk

James Dombrowski (Genetics) N. Raikhel

Susan Fujimoto* (Genetics) from September 16, 1990

Pedro Gil (Botany and Plant Pathology) P. Green from March 16, 1990

Carrie Hiser (Biochemisiry) L. Mclntosh

Susanne Hoffmann (Genetics) H. Kende

Christie Howard (Biochemistry) P. Green from June 16, 1990

Mary Kakefuda (Genetics) L. Mclntosh

Ellen Kearns (Genetics) C. Somerville

Hyung-Il Lee (Genetics) N. Raikhel

David Lerner (Botany and Plant Pathology) N. Raikhel

Robert Meeley (Biochemistry) J. Walton

Anastasia Nikolskaya* (Botany and Plant Pathology) from September 16, 1990

Vladimir Orbovic (Botany and Plant Pathology) K. Poff

Scott Peck (Botany and Plant Pathology) H. Kende

David Rhoads (Biochemistry) L. Mcintosh

Christopher Rock (Genetics) J. Zeevaart

Elizabeth Rosen (Botany and Plant Pathology) K. Poff from June 16, 1990

Holly Schaeffer (Genetics) J. Walton 
Mark Shieh (Botany and Plant Pathology) N. Raikhel

Samuel Simons (Blochemistry) S. Somerville

Lawrence Smart (Genetics) L. Mclntosh

Crispin Taylor (Genetics) P. Green

Sharon Thoma (Botany and Plant Pathology) C. Somerville

Jun Tsuji (Genetics) S. Somerville

Frank van de Loo (Botany and Plant Pathology) C. Somerville

Ambrosius van Hoff* (Genetics) from September 16, 1990

Ronda White (Genetics) H. Kende

Ruth Wilson (Genetics) C. Somerville

*Students who had not yet chosen their supervising faculty member

Technical Personnel (Including Supervising Faculty Member; if none listed -general service)

Thérèse Best, Research Assistant II (K. Poff)

Lisa Carlson, Laboratory Research Technician I (S. Somerville) until June 6, 1990 Linda Danhof, Research Specialist (C. Somerville)

Renate de Zacks, Laboratory Research Technician II (H. Kende and D. Lamport) Robert Geyer, Instrument Maker II

Margaret Graham, Laboratory Worker, from January 31, 1990

Frank Holden, Laboratory Research Technician I (J.D. Walton) until January 3 , 1990

Samuel Jackson, Instrument Maker I, until July 20, 1990

Robert Kever, Equipment Mechanic

Amy Klecan, Research Specialist (S. Somerville), until March 26, 1990

Elliot Light, Greenhouse Coordinator

Roxy Nickels, Research Assistant II (L. Mclntosh)

Elaine Oren, Laboratory Research Technician II (C.P. Wolk) from June 1, 1990 until September 28, 1990

Kelly Owens, Laboratory Research Technician II (C.P. Wolk) until June 5, 1990 Maria Schneider, Research Specialist (F. de Bruijn) from July 1, 1990

Anna Summers, Laboratory Worker until January 31, 1990

\section{Administrative and Clerical Personnel}

Alice Albin, Administrative Assistant III

Karen Cline, Administrative Assistant II

Janet Johnson, Typist Technical

Maxine Lance, Typist Technical

Jacqueline Malkin, Account Clerk II 


\section{OUTSIDE FUNDING SOURCES FOR PRL PERSONNEL}

The Plant Research Laboratory faculty and research staff has been successful in obtaining individual research support from extramural, and also intramural sources. Research grants which supported personnel during 1990 are listed below according to faculty member. Pre- and postdoctoral personnel who were on fellowships or other types of individual funding are listed in the second section.

\section{PRL Faculty}

Frans de Bruijn - USDA, MSU Center for Microbial Ecology

Pamela Green - USDA, McKnight Foundation, Pioneer HI-Bred

Andrew Hanson - NSF, USDA, CIBA-GEIGY, MSU Agricultural Experiment Station, MSU Research Excellence Funds, Rockefeller Foundation, Sugar Beet Foundation

Hans Kende - NSF, USDA

Derek T.A. Lamport - NSF, USDA, BARD, MSU Research Excellence Funds, PEPSICO

Lee McIntosh - NSF, USDA, BARD, CIBA-GEIGY Corporation, McKnight Foundation, MSU Agricultural Experiment Station, MSU Research Excellence Funds, Plant Genetics Systems Inc., Rackham

Kenneth L. Poff - NASA, Shell

Natasha Raikhel - NSF, MSU Biotechnology Research Center, MSU Research Excellence Funds, NATO

Christopher R. Somerville - DuPont Company, Eli Lilly Company, McKnight Foundation, MSU Biotechnology Research Center, MSU Research Excellence Fund, NSF, Pioneer International, USDA, USDA/NSF/DOE Plant Science Center

Shauna C. Somerville - NIH, USDA, MSU Agricultural Experiment Station, MSU AURIG, MSU Research Excellence Funds

Jonathan D. Walton - NSF, MSU Bioresearch Center, MSU Research Excellence Funds, NATO

C. Peter Wolk - NSF, MSU Center for Microbial Ecology, MSU Research Excellence Funds

Jan A.D. Zeevaart - NSF, USDA 


\section{Individual Fellowships and Funding}

Shawn Anderson - MSU College of Natural Science Fellowshlp

Vincent Arondel - European Molecular Blology Organization

Neil Bowlby - NSF Postdoctoral Fellowship

Bertha Bullen - MSU College of Natural Science Fellowship, NASA Predoctoral Fellowship

Clinton Chapple - Natural Science and Engineering Council of Canada

Therese Duggelin - Swiss National Science Foundation

Anneliese Ernst - Deutsche Forschungsgemeinschaft

Marie-Claude Fortin - Agriculture Canada Research

Susan Gibson - NIH Postdoctoral Fellowship

Pedro Gil - Spanish Ministry of Agriculture

Erwin Grill - Deutsche Forschungsgemeinschaft

Ursula Hecht - Lynen Fellowship

Carrie Hiser - MSU College of Natural Science Fellowship

Ellen Kearns - MSU Biotechnology Fellowship

Bertrand Lemieux - Canadian NSERC Fellowship

David Lerner - MSU Biotechnology Fellowship

Taka-Aki Ono - U.S. - Japan Program of Cooperation on Photoconversion and Photosynthesis

Yves Poirier - Canadian NSERC Fellowship

Wolf-Dieter Reiter - Max Planck Society, Otto-Hahn-Medal Award

Christopher Rock - MSU College of Natural Science Fellowship 
Margret Sauter - Max Kade Foundation Postdoctoral Fellowship

Jane Schneider - NSF Postdoctoral Fellowship

Martin Schroeder - Deutsche Forchungsgemeinschaft

Lawrence Smart - MSU Biotechnology Fellowship

Jun Tsuji - MSU College of Natural Science Fellowship 


\section{PUBLICATIONS}

Bednarek, S.Y., T.A. Wilkins, J.E. Dombrowski and N.V. Raikhel: A carboxylterminal propeptide is necessary for proper sorting of barley lectin to vacuoles of tobacco. The Plant Cell 2:1145 1155 (1990).

Bensen, R.J. and J.A.D. Zeevaart: Comparison of ent-kaurene synthetase $A$ and $B$ activities in cell-free extracts from young tomato fruits of wild-type and gib-1, gib-2, and gib-3 tomato plants. J. Plant Growth Regul. 9:237-242 (1990).

Bernhard, W.R., S. Thoma, J. Botella and C.R. Somerville: Isolation of a CDNA clone for spinach lipid transfer protein and evicenics that the protein is synthesized by the secritory pathway. Plant $P h$, si:J. 95:164-170 (1991).

Briggs, L.M., V.L. Pecoraro and L. McIntosh: Copper-induced expression and regulatory studies of the plastocyanin gene from the cyanobacterium Synechocystis sp. PCC 6803. Plant Molec. Biol. 15:633-642 (1990).

Broekaert, W., H.-L. Lee, A. Kush, N.-H. Chua and N. Raikhel: Wound-induced accumulation of mRNA containing a hevein sequence in laticifers of rubber tree (Hevea brasiliensis). Proc. Natl. Acad. Sci. USA 87:7633-7637 (1990).

Brouquisse, R., P. Weirel, D. Rhodes, C.F. Yocum and A.D. Hanson: Evidence for a ferredoxin-dependent choline monooxygenase from spinach chloroplast stroma. Plant Physiol 90:322-329 (1989).

Bullen, B.L., T.R. Best, M.M. Gregg, S.-E. Barsel and K.L. Poff: A direct screening procedure for gravitropism mutants in Arabidopsis thaliana (L.) Heynh. Plant Physiol. 93:525-531 (1990).

Cai, Y. and C.P. Wolk: Use of a conditionally lethally gene in Anabaena sp. strain PCC i120 to select for double recombinants and to entrap insertion sequences. J. Bacteriol. 172:3138-3145 (1990).

Chrispeels, M.J. and N.V. Raikhel: Lectins, lectin genes, and their role in plant defense. The Plant Cell 3:1-9 (1991).

de Bruijn, F.J., L. Szabados and J. Schell: Chimeric genes and transgenic plants are used to study the regulation of genes involved in symbiotic plant-microbe interactions (nodulin genes). Development. Genet. 11:182-196 (1990). 
de Bruijn, F.J., U. Hilgert, J. Stigter, M. Schneider, H. Meyer Z.A., U. Klosse and K. Pawiowski: Regulation of nitrogen fixation and assimilation genes in the freeIiving versus symbiotic state. In: Nitrogen fixation: Achievements and Objectives, (P. Gresshoff, L.E. Roth, G. Stacey and W.E. Newton, eds.), pp. 33-44, Chapman and Hall, New York and London (1990).

Delmer, D.P.: Role of the plasma membrane in cellulose synthesis. In: The Plant Plasma Membrane (C.Larsson and I.M. Moller, eds.), pp. 256-268, SpringerVeriag, Berlin and Heidelberg (1990).

Edelman, L. and H. Kende: A comparison of 1-aminocyclopropane-1-carboxylate synthase in vitro translation product and in-vivo-labeled protein in ripening tcmatoes. Planta 182:635-638 (1990).

Elhai, J. and C.P. Wolk: Developmental regulation and spatial pattern of expression of the structural genes for nitrogenase in the cyanobacterium Anabaena. EMBO J. 9:3379-3388 (1990).

Elhai, J., T. Thiel and H.B. Pakrasi: DNA transfer into cyanobacteria. Plant Molec. Biol. Manual A12:1-23, Kluwer Åcademic Publ., Dordrecht (1990).

Elthon, T.E., R.L. Nickels and L. Mclntosh: Mitochondrial events during development of thermogenesis in Sauromatum guttatum (Schott). Planta 180:8289 (1989).

Finkelstein, R.R. and C.R. Somerville: Three classes of abscisic acid (ABA)insensitive mutations of Arabidopsis define genes that control overlapping subsets of ABA responses. Plant Physiol. 94:1172-1179 (1990).

Fitchen, J.H., S. Knight, I. Andersson, C.-I. Branden and L. Mclntosh: Residues in three conserved regions of the small subunit of ribulose-1,5-bisphosphate carboxylase/oxygenase are required for quaternary structure. Proc. Natl. Acad. Sci. USA 87:5768-5772 (1990).

Fortin, M.-C.A. and K.L. Poff: Temperature sensing by primary roots of maize. Plant Physiol. 94:367-369 (1990).

Hanson, A.D.: Drought resistance in rice. Nature 345:26-27 (1990).

Haughn, G.W. and C.R. Somenille: A mutation causing imidazolinone resistance maps to the Csr1 locus of Arabidopsis thaliana. Plant Physiol. 92:1081-1085 (1990). 
Heath, T.G., D.A. Gage, J.A.D. Zeevaart and J.T. Watson: Role of molecular oxygen in fragmentation processes of abscisic acid methyl ester in electron capture negative ionization. Organic Mass Spec. 25:655-663 (1990).

Hiser, C. and L. Mclntosh: Alternative oxidase of potato is an integral membrane protein synthesized de novo during aging of tuber slices. Plant Physiol. 93:312. 318 (1990).

Holland, D. and C.P. Wolk: Identification and characterization of hetA, a gene that acts early in the process of morphological differentiation of heterocysts. J. Bacteriol. 172:3131-3137 (1990).

Hondred, D. and A.D. Hanson: Hypoxically inducible barley lactate dehydrogenase: CDNA cloning and molecular analysis. Proc. Natl. Acad. Sci. USA 87:7300-7304 (1990).

Hugly, S., P. McCourt, J. Browse, G.W. Patterson and C. Somerville: A chilling sensitive mutant of Arabidopsis with altered steryl-ester metabolism. Plant Physiol. 93:1053-1062 (1990).

Janoudi, A. and K.L. Poff: A common fluence threshold .ur first positive and second positive phototropism in Arabidopsis thaliana. Plant Physiol. 94:16051608 (1990).

Janoudi, A. and K.L. Poff: Characterization of adaptation in phototropism of Arabidopsis thaliana. Plant Physiol. 95:517-521 (1991).

Kieliszewski, M.J., J.F. Leykam and D.T.A. Lamport: Structure of the threoninerich extensin from Zea mays. Plant Physiol. 92:316-326 (1990).

Klecan, A.L., S. Hippe and S.C. Somerville: Reduced growth of Erysiphe graminis f.sp. hordei induced by Tilletiopsis pallescens. Phytopath. 80:325-331 (1990).

Konjevic, R., B. Steinitz and K.L. Poff: Dependence of the phototropic response of Arabidopsis thaliana on fluence rate and wavelength. Proc. Natl. Acad. Sci. USA 86:9876-9880 (1990).

Lemieux, B., M. Miquel, C. Somerville and J. Browse: Mutants of Arabidopsis with alterations in seed lipid fatty acid composition. Theor. Appl. Genet. 80:234240 (1990).

Mansfield, M.A. and N.V. Raikhel: Abscisic acid enhances the transcription of wheat-germ agglutinin mRNA without altering its tissue-specific expression. Planta 180:548-554 (1990). 
Martinez-Zapater, J.M. and C.R. Somerville: Effect of light quality and vernalization on late-flowering mutants of Arabidopsis thaliana. Plant Physiol. 92:770-776 (1990).

McCue, K.F. and A.D. Hanson: Drought and salt tolerance: towards understanding and applications. Trends in Biotechnology 8:358-362 (1990).

Moffatt, B.A. and C.R. Somerville: Purification of adenine phosphoribosyltransferase from Brassica juncea. Arch. Biochem. Biophys. 283:484-490 (1990).

Read, S.M. and D.P. Delmer: Biochemistry and regulation of cellulose synthesis in higher plants. In: Biosynthesis and Biodegradation of Cellulose (C.H. Haigler and P.J. Weimer, eds.), pp. 177-200, Marcel Dekker, Inc., New York, Basel and Hong Kong (1991).

Rock, CD. and I.A.D. Zeevaart: Abscisic (ABA)-aldehyde is a precursor to, and $1^{\prime}, 4^{\prime}$-trans-ABA-did a catabolite of, ABA in apple. Plant Physiol. 93:915-923 (1990).

Schaeffer, H.J. and J.D. Walton: Aluminum ions induce oat protoplasts to produce an extracellular (1-3)B-D-glucan. Plant Physiol. 94:13-19 (1990).

Schiefelbein, J.W. and C.R. Somerville: Genetic control of root hair development in Arabidopsis thaliana. The Plant Cell 2:235-243 (1990).

Scott-Craig, J.S., D.G. Palraccione, F. Cervone and J.D. Walton: Endopolygalacturonase is not required for pathogenicity of Cochliobolus carbonum on maize. The Plant Cell 2:1191-1_00 (1990).

Shedletzky, E., M. Shmuel, D.P. Delmer and D.T.A. Lamport: Adaptation and growth of tomato cells on the herbicide 2,6-dichlorobenzonitrile leads to production of unique cell wails virtually lacking a cellulose-xyloglucan network. Plant Physiol. 94:980-98:' (1990).

Sherwood, J.E. and S.C. Somerville: Sequence of the Erysiphe graminis f. sp. hordei gene encoding B-tubulin. Nucl. Acids Res. 18:1052 (1990).

Somerville, C.R.: The biochemical basis for plant improvement. In: ?lant Physiology, Biochemistry and Molecular Biology (D.T. Dennis and D.H. Turpin, eds.), pp. 490-501, Longman, Harlow, U.K. (1990).

Szabados, L., P. Ratet, B. Grunenberg and F.J. de Bruijn: Functional analysis of the Sesbania rostrata leghemoglobin glb3 gene 5'-upstream region in transgenic Lotu's corniculatus and Nicotiana tabacum plants. The Plant Cell 2:973-986 (1990). 
Talon, M., M. Koornneef and J.A.D. Zeevaart: Accumulation of $\mathrm{C}_{99}$-gibberellins in the gibberellin-insensitive dwarf mutant gai of Arabidopsis thaliana (L.) Heynh. Planta 182:501-505 (1990).

Talon, M., M. Koornneef and J.A.D. Zeevaart: Endogenous gibberellins in Arabidopsis thaliana and possible steps blocked in the biosynthetic pathways of the semidwarf ga4 and ga5 mutants. Proc. Natl. Acad. Sci. USA 87:7983-7987 (1990).

Talon, M. and J.A.D. Zeevaart: Gibberellins and stem growth as related to photoperiod in Silene armeria L. Plant Physiol. 92:1094-1100 (1990).

Walton, J.D.: Genetics and biochemistry of toxin synthesis in Cochliobolus (Helminthosporium). In: Molecular Industrial Mycology (S.A. Leong and R.M. Berka, eds.), pp. 225-249, Marcel Dekker, New York (1990).

Walton, J.D.: Peptide phytotoxins from plant pathogenic fungi. In: Biochemistry of Peptide Antibiotics (H. Kleinkauf and H. von Döhren, eds.), pp. 179-203, Walter de Gruyter, Berlin (1990).

Walton, J.D. and F. Cervone: Endopolygalacturonase from the maize pathogen Cochliobolus carbonum. Physiol. Mol. Plant Pathol. 36:351-359 (1990).

Weretilnyk, E.A. and A.D. Hanson: Molecular cloning of a plant betaine-aldehyde dehydrogenase, an enzyme implicated in adaptation to salinity and drought. Proc. Natl. Acad. Sci. USA 98:2745-2749 (1990).

White, J.A. and H. Kende: 1-Aminocyclopropane-1-carboxylate synthase: are there charge and size variants in tomato? J. Plant Physiol. 136:646-652 (1990).

Wilkins, T.A., S.Y. Bednarek and N.V. Raikhel: Role of propeptide in posttranslational processing and transport of barley lectin to vacuoles in transgenic tobacco. The Plant Cell 2:301-313 (1990).

Xiong-biao L., M. Kieliszewski and D.T.A. Lamport: A chenopod extensin lacks repetitive tetrahydroxyproline blocks. Plant Physiol. 92:327-333 (1990).

Zeevaart, J.A.D., D.A. Gage and R.A. Creelman: Recent studies of the metabolism of abscisic acid. In: Plant Growth Substances, 13th Intern. Conf. on Plant Growth Substances, Calgary, 1988 (R.P. Pharis and S.B. Rood, eds.), pp. 233-240, Springer, Berlin (1990). 
Zeevaart, J.A.D., M. Talon and T.M. Wilson: Stem growth and gibberellin metabolism in relation to photoperiod. In: Gibberellin Symposium Tokyo 1989, (N. Takahashi, J. MacMillan and B.O. Phinney, eds.), pp. 273-279, Springer, Berlin (1990).

Zhang, $\mathrm{H}$. and C. Somerville: Soluble and membrane-bound forms of cytochrome $b_{3}$ are the products of a single gene in chicken. Arch. Biochem. Biophys. 280:412-415 (1990). 


\section{PAPERS PRESENTED AT MEETINGS}

(*denotes speaker)

International Congress of Pacific Basin Chemical Societies, Honolulu, Hawaii (January 1990). Walton, J.D. *, "Biochemistry of fungal cyclic peptide synthesis."

University of California Symposium on Molecular and Cellular Biology: Genetic and In Vitro Analysis of Cell Compartmentalization, Taos, New Mexico (February 1990). Bednarek, S.* and N. Raikhel, "Sorting mechanisms of barley lectin to the vacuoles of plant cells."

Society for Experimental Biology, Warwick, United Kingdom (March 1990). Hanson, A.D.*, "Enzymes and genes of the betaine pathway in plants."

National Science Foundation United States Japan Symposium on Photosynthesis, Honolulu, Hawaii (April 1990). Mclntosh, L.*, "Protein engineering of PS II."

University of California Symposium on Crop Improvement, Keystone, Colorado (April 1990). Somerville, C.R. ${ }^{\star}$, "Genetic manipulation of oil composition", Kearns, E.* and C.R. Somerville, "The role of cytochrome $b_{5} \Delta-12$ desaturation by microsomes of Carthamus tinctorus"; Raikhel, N. *, "Role of glycan in processing and transport of chitin-binding lectin of barley in transgenic tobacco", and "Cloning of a cDNA expressed in laticifers of rubber tree containing hevein amino acid sequence"; Kende, $H .{ }^{*}$, "Control of ethylene biosynthesis."

American Society of Clinical Nutrition, Washington, D.C. (April 1990). Somerville, C.R. ${ }^{\star}$, "Genetic engineering of plant lipid composition."

Cold Spring Harbor Banbury Symposium, Cold Spring Harbor, New York (April 1990). Walton, J.D.", "Molecular cloning of the TOX locus of Cochliobolus carbonum."

Joint FAO/IAEA United Nations Meeting, Vienna, Austria (May 1990). Thomashow, S." and C.R. Somenille, "Strategies for the manipulation of lipid biosynthesis"; Somerville, C.R.* "Why such a thin plant in such a fat lab."

National Meeting of American Society of Microbiology, Anaheim, California (May 1990). Wolk, C.P^, "Mapping Anabaena, a differentiating bacterium that performs oxygenic photosynthesis."

MSU-DOE Plant Research Laboratory 25th Anniversary Symposium, East Lansing, Michigan (May 1990). Delmer, D. *, "The plant cell wall as a dynamic structure." 
American Society of Cell Biology Summer Research Conference on the Biology of the Plant and Animal Matrix, Airlie, Virginia (June 1990). Delmer, D*., "Biosynthesis and assembly of cellulose and non-cellulosic beta-glucans."

American Society of Cell Biology, Airlie, Virginia (June 1990). Kieliszewski, M. and Lamport, D.T.A.*, "Evolution of a cell wall model."

Gordon Conference on Plant Molecular Biology, Andover, New Hampshire (June 1990). Kende, $H_{.}^{*}$, "Transduction mechanisms involving ethylene"; Heese, $A .{ }^{*}$, "Cell and signals"; Raikhel, N.*, "Protein targeting to the vacuole."

2nd International Symposium: Plant-Soil Interactions at Low pH, Beckley, West Virginia (June 1990). Schaeffer, H. * and J.D. Walton, "Aluminum ions induce oat protoplasts to produce an extra cellular B-(1-3)-glucan."

Australian Gene Mapping Workshop, Sydney, Australia (June 1990). Gibson, S.* and C.R. Somerville, "Construction of a YAC library and physical mapping of the genome of the higher plant, Arabidopsis thaliana."

Genetics Society Meeting of Australia, Adelaide, Australia (June 1990). Gibson, S. ${ }^{*}$ and C.R. Somerville, "Construction and use of a yeast artificial chromosome library to isolate fatty acid desaturase genes from the higher plant, Arabidopsis thaliana."

4th. International Arabidopsis Meeting, Vienna, Austria (June 1990). Somerville, C.R.", "Why such a thin plant in such a fat lab."

Gordon Conference on Fungal Metabolism, Andover, New Hampshire (July 1990). Walton, J.D. *, "Biology of toxin biosynthesis."

Gordon Conference, New London, New Hampshire (July 1990). Mclntosh, L. *, "The structure and function of the alternative oxidase."

9th International Symposium on the Biochemistry, Structure and Utilization of Plant Lipids, Wye, Kent, United Kingdom (July 1990). Shanklin, J.* and C.R. Somerville, "Characterization of stearoyl-ACP desaturase"; Thoma, S. ${ }^{\star}$ and C.R. Somerville, "Spinach lipid transfer protein is synthesized by the secretory pathway"; Somerville, C.R.*, "Methods for isolation of genes involved in lipid metabolism."

World Hydrogen Energy Conference \#8, Kailua-kona, Hawaii (July 1990). Wolk, C.P. ${ }^{\star}$, J. Elhai, Y. Cai and I. Bancroft, "Genetic engineering of the cyanobacterium Anabaena." 
Hydrogen Production Workshop N Following World Hydrogen Energy Conference \#8, Kailua-kona, Hawaii (July 1990). Wolk, C.P.*, "Genetics of Anabaena."

Annual Meeting of American Society of Plant Physiologists, Indianapolis, Indiana (July 1990). White, J.* and $H$. Kende, "Isoelectric focusing of wound-induced tomato ACC synthase"; Sauter, $M .{ }^{*}$ and $H$. Kende, "Lignification in rapidly growing internodes of deepwater rice"; Hoffmann, S." and H. Kende. "The role of ABA in the growth of rice coleoptiles"; Schaeffer, H.* and J.D. Walton, "Victorin-induced extracellular B-(1-3)-glucan in oat protoplasts"; Hanson, A.D.*, C. Lerma, J. Bolanos and D. Rhodes, "Betaine deficiency in maize: metabolic basis and relation to osmotic adjustment"; Orbovic, V. * and K. Poff, "An infra-red imaging system for the analysis of tropisms in A. thaliana seedlings"; Kakefuda, M.* and L. Mclntosh, "Expression of alternative oxidase in tomato"; Hiser, C. * and L. Mclntosh, "Cloning and expression of the alternative oxidase gene of potato"; Smart, L.* and L. Mclntosh, "Regulation of genes encoding PSI and PSII proteins in Synechocystis PCC 6803"; Sithole, I.", N. Bowlby, G.T. Babcock and L. Mclntosh, "A preliminary biophysical analysis of site-specific mutations in the photosystem II reaction center of the cyanobacterium Synechocystis 6803"; Anderson, S.* and L. Mclntosh, "Cloning and gene arrangement of psaC, ndhE and ndhD from the cyanobacterium Synechocystis sp. PCC 6803"; Rhoads, D.* and L. Mclntosh, "Isolation and analysis of a cDNA clone encoding an S. guttatum alternative oxidase protein"; Benbow, L." and D.T.A. Lamport, "Asparagus HRGP(s): The primary cell wall of a non-graminaceous monocot"; Meeley, R. ${ }^{\star}$ and J.D. Walton, "Metabolism of the cyclic peptide HC-toxin, a host-specific toxin, by resistant and susceptible maize"; Simons, S. ${ }^{\star}$ and S.C. Somerville, "Differential protein in barley cultivars resistant or susceptible to E. graminis horder"; McClie, K.* and A.D. Hanson, "CDNA cloning and analysis of $B A D H$, a salt inducible enzyme in sugar beet"; Kieliszewski, M.* and D.T.A. Lamport, "Two histidine-rich HRGPs from Zea mays"; Bensen, R. * and J.A.D. Zeevaart, "Comparison of ent-kaurene synthetase $A$ and $B$ activities in cell-free extracts from young tomato fruits of wild-type and dwarf gib-1 and gib-3 tomato plants"; Rock, C." and J.A.D. Zeevaart, "ABA alcohol is an intermediate in abscisic acid biosynthesis"; Talon, M.* and J.A.D. Zeevaart, "Characterization of dwarf GA mutants of Arabidopsis"; Benning, C.* and C.R. Somerville, "Analysis of the biosynthetic pathway for sulfoquinovosyl-diacylglycerol in the purple bacterium $R$. sphaeroides"; Hoffmann, S." and H. Kende, "The role of $A B A$ in the growth of rice coleoptiles"; Delmer, D.*, "DCB-adapted cells possess unique wall structure"; and "Cation-induced formation of a macro-glucan synthesis complex."

American Phytopathological Society and Canadian Phytopathological Meeting, Grand Rapids, Michigan (August 1990). Tsuji, J." and S. Somerville, "Phytoalexin accumulation in Arabidopsis thaliana leaves inoculated with Pseudomonas syringae pv. syringae"; Scott-Craig, J." and J.D. Walton, "Molecular cloning and characterization of an endopolygalacturonase gene from Cochliobolus carbonum"; Walton, J.D.* J.S. Scott-Craig and J.-A. Pocard, "Molecular cloning 
of the TOX locus from Cochliobolus carbonum"; Panaccione, D. ${ }^{\star}$, J.S. ScottCraig, S, J.-A. Pocard and J.D. Walton, "Characterization of the TOX locus of Cochliobolus carbonum"; Somerville, S.C.", A.L. Klecan and S. Hippe, "Reduced growth of Erysiphe graminis f. sp. hordei induced by Tilletiopsis pallescens."

7th Congress of Federation of European Societies of Plant Physiology, Umea, Sweden (August 1990). Zeevaart, J.A.D.*, "Hormonal signals in flowering."

Plant Growth Regulator Society of America Meeting, St. Paul, Minnesota (August 1990). Bensen, R.* and J.A.D. Zeevaart, "Characterization of five Arabidopsis dwarf mutants which are gibberellin deficient."

4th International Workshop on Plant Mitochondria, Ithaca, New York (September 1990). Rhoads, D.* and L. Mclntosh, "Isolation and analysis of a cDNA clone encoding an S. guttatum alternative oxidase protein"; Hiser, C. ${ }^{\star}$ and L. Mclntosh, "Alternative oxidase expression in aging potato tuber slices"; Mclntosh, L., "Alternative oxidase of plant mitochondria."

International Conference on Comparative Physiology, Crans-sur-Sierre, Switzerland (September 1990). Hanson, A.D. *, "Compatible solute synthesis and compartmentation."

International Symposium on Molecular Genetics of Plant Microbe Interactions, Interlaken, Switzerland (September 1990). Somerville, S.* and J.E. Sherwood, "Mutational analysis of host-pathogen interactions in the barley powdery mildew disease."

International Symposium on Plant Molecular Biology and Biotechnology, Sydney, Australia (September 1990). de Bruijn, F.*, "Regulation of plant genes specifically induced in nitrogen-fixing nodules."

American Society of Gravitational and Space Biology, Louisville, Kentucky (November 1990). Poff, K.L. *, "Gravitropism and phototropism in Arabidopsis."

National Cell Biology Meeting, San Diego, California (December 1990). Raikhel, $N .{ }^{\star}$, "A carboxyl-terminal propeptide is necessary for proper sorting of barley lectin to vacuoles of tobacco."

Keystone Symposium on Dissection of Plant Cell Processes, Keystone, Colorado (January 1991). Somerville, C.*, "Genetics of plant lipid metabolism"; McCue, K.* and A.D. Hanson, "Expression and analysis of betaine aldehyde dehydrogenase, a salt inducible enzyme cloned from sugar beet (Beta vulgaris)"; Delmer, D.*, "Identification and characterization of genes and gene products involved in betaglucan synthesis." 
Gordon Conference on Temperature Stress in Plants, Oxnard, California (January 1991). Schneider, C.J." and C.R. Somerville, "Chilling sensitive mutants of Arabidopsis"; Thomashow, S." and C.R. Somerville, "Responses of lipid mutants of Arabidopsis to chilling temperatures." 


\section{SEMINAR SCHEDULE}

Speaker

\section{WINTER TERM}

Dr. Alan M. Jones

University of North Carolina

Chapel Hill, North Carolina

Dr. Steven Kay

The Rockefeller University

Now York, New York

Dr. Kenneth Keegstra

University of Wisconsin

Madison, Wisconsin

Dr. Michael Thomashow*

Michigan State University

East Lansing, Michigan

Dr. Fred D. Sack

Ohio State University

Columbus, Ohio

Dr. William J. Lucas

University of California

Davis, California

Dr. Richard D. Vierstra

University of Wisconsin

Madison, Wisconsin

Dr. David T. Dennis

Queens University

Kingston, Ontario, Canada
Topic and Date

"Light regulation of maize mesocotyl growth: The role of auxin and a putative auxin receptor"

January 10, 1990

The analysis of phytochrome functions in transgenic plants" January 17, 1990

"Transport of proteins into the chloroplast"

January 24, 1990

"Molecular genetics of cold acclimation in Arabidopsis"

January 31, 1990

"Heavy bodies and circumstance: do amyloplasts "still" function in in gravity sensing"

February 7, 1990

"Plasmodesmata: structure, function and viral interaction"

February 14, 1990

"How do cells turn over proteins?"

February 21, 1990

"Biochemical and molecular aspects of the compartmentation of metabolism in plants"

February 28, 1990 
Dr. Joseph Berry

Carnegie Insttution of Washington

Stanford, Californla

Dr. Steven Knapp

Oregon State University

Convallis, Oregon
"Regulation of canopy transpiration and photosynthesis by stomata: an empirical model" March 7, 1990

"Cuphea viscosissima: a new crop and model system for investigating medium chain fatty acid symthesis in developing plant seeds"

March 12, 1990

\section{SPRING TERM}

Dr. Robert Fischer University of California

Berkeley, California

Dr. Wilbur Campbell

Michigan Technological University

Houghton, Michigan

Dr. Herman van den Ende

University of Amsterdam

Amsterdam, The Netherlands

Dr. Mary Schuler

University of Illinois

Urbana, Illinois

Dr. Winslow Briggs

Carnegie Institution of Washington

Stanford, California

Dr. Mary Jane Saunders

cell University of South Florida

Tampa, Florida

Dr. John Abelson ${ }^{\star \star}$

California Institute of Technology

Pasadena, California
"Regulation of tomato fruit ripening"

March 28, 1990

"Biochemistry and molecular biology of higher plant nitrate reductases"

April 4, 1990

"Sexual signaling in Chlamydomonas"

April 11, 1990

"Plant pre-mRNA splicing" April 18, 1990

"Evidence for two photoreceptors in pea plasma membranes" April 25, 1990

"Calcium, cytokinin and division in plants"

May 2, 1990

"RNA splicing in yeast"

May 9, 1990 
Dr. Frederick Ausubel\#

Massachusetts General Hospital

Boston, Massachusetts

Dr. Randy Schekman

University of California

Berkeley, California

Dr. Dieter Klämbt

University of Bonn

Bonn, West Germany

Dr. Peter van den Elzen

Mogen International nv

The Netherlands

\section{FALL TERM}

Dr. Grattan Roughan Mount Albert Research Center

Auckland, New Zealand

Dr. Vincenzo De Luca

Université de Montréal

Institut Botanique

Montréal, Québec, Canada

Dr. Paul Staswick

University of Nebraska

Lincoln, Nebraska

Dr. Joe Chappell

University of Kentucky

Lexington, Kentucky

Dr. Ching Kung

University of Wisconsin

Madison, Wisconsin
"Bacterial pathogenesis of Arabidopsis thaliana"

May 15, 1990

"Molecular components required for protein translocation into endoplasmic reticulum"

May 16, 1990

"The function of the auxin-binding protein from maize coleoptiles" June 8, 1990

"Pathogenesis-related genes and their expression in tobacco" June 14, 1990

The role of chloroplasts in leaf lipid metabolism"

September 26, 1990

"Molecular approaches to altering secondary metabolism in plants" October 3, 1990

"Physiological signals and molecular control of the expression of soybean vegetative storage protein genes"

October 10, 1990

"Rate-limiting steps, signals and other secrets of sesquiterpene metabolism in plants" October 17, 1990

"Ion channels of Paramecium, yeast, and $E$. coli"

October 24, 1990 
Dr. Sarah Assmann

Harvard University

Cambridge, Massachusetts

Dr. David Oliver

Universitv of Idaho

Moscow, Idaho

Dr. Richard Dixon

The S.R. Noble Foundation

Ardmore, Oklahoma
"Blue light regulation of stomatal opening: studies using gas exchange and patch clamp techniques"

October 31, 1990

"Glycine metabolism by leaf mitochondria: developmentaland molecular biology of the glycine decarboxylase multi-enzyme complex"

November 7, 1990

"Molecular genetic aspects of plant secondary metabolism in relation to defense"

November 14, 1990

\section{WINTER TERM}

Dr. Jan A.D. Zeevaart

MSU-DOE Plant Research Lab

Michigan State University

East Lansing, Michigan

Dr. Charles Yocum

University of Michigan

Ann Arbor, Michigan

Dr. Richard Broglie\#\#

DuPont Experiment Station

Wilmington, Delaware

Dr. Peter Gresshoff

University of Tennessee

Knoxville, Tennessee

"Biosynthetic pathways of abscisic acid and gibberellin in higher plants" January 9, 1991

"Probing calcium function in photosynthetic $\mathrm{O}_{2}$ evolution" January 16, 1991

"Molecular approaches to fungal dissase resistance" January 22, 1991

"Molecular genetic analysis of nodulation control in soybean" January 30, 1991

*Cosponsored with the Department of Botany and Plant Pathology

**Special Barry Chelm Memorial Lecture, cosponsored with the Department of Microbiology and Public Health

\#Cosponsored with the Department of Genetics

\#\#Cosponsored by Monsanto and the Department of Horticulture 


\section{$25^{\text {th }}$ ANNIVERSARY SYMPOSIUM \\ May 25 - 26, 1990}

\section{PROGRAM}

\section{GLOBAL ASPECTS OF PLANT BIOLOGY}

Robert Rabson (Department of Energy, Division of Energy Biosciences) Introduction

John W. Mellor (International Food Policy Research Institute, Washington DC) "Plant Biology in a World Without Hunger: Getting There and Staying There"

Peter S. Ashton (Harvard University, Cambridge, MA)

"Biological Challenges Behind the Biodiversity Crisis"

Leslie Fowden (Rothamsted Experimental Station, Harpenden, U.K.)

"The Prospect for Greater Diversity in the Future Utilization of Plants"

\section{ENERGY CAPTURE AND PHOTOSYNTHESIS}

Hartmut Michel (Max-Planck-Institut für Biophysik, Frankfurt, FRG)

"The Photosynthetic Reaction Center from Purple Bacteria and its Relation to Photosystem II"

Charles J. Arntzen (Texas A\&M University, College Station, TX)

"Structure-Function Reilationship of Chloroplast Membrane Proteins"

Cart-Ivar Brändén (Swedish University of Agricultural Sciences, Uppsala, Sweden) "Photosynthesis: Energy Capture and Conversion"

William L. Ogren (USDA/University of Illinois, Urbana, IL)

"Rubisco Regulation of Photosynthetic Productivity"

\section{PLANTS AND STRESS}

John S. Boyer (University of Delaware, Lewes, DE)

"Water and Plant Growth"

Hans J. Bohnert (University of Arizona, Tucson, AZ)

"Molecular Aspects of Induction of CAM by Osmotic Stress"

Clarence A. Ryan (Washington State University, Pullman, WA)

The Regulation and Roles of Proteinase Inhibitor Genes in Natural Defense Systems" 


\section{PLANT DEVELOPMENT}

Deborah P. Delmer (Hebrew University, Jerusalem, Israel)

The Plant Cell Wall as a Dynamic Structure*

Peter H. Quail (University of California Berkeley/USDA Plant Gene Expression Center, Albany, CA)

"Phytochrome and Signal Transduction in Plants"

Adrienne E. Clarke (University of Melbourne, Parkville, Victoria, Australia)

"Molecular Genetics of Self-Incompatibility in Flowering Plants"

Jozef S. Schell (Max-Planck-institut für Züchtungsforschung, Köln, FRG)

"The Study of Genes Involved in the Control of Plant Development"

\section{SUMMARY LECTURES}

Uoyd T. Evans (CSIRO - Division of Plant Industry, Canberra, Australia) "Postponing the Plateau in Crop Yields: Is Plant Physiology Still Needed?"

Mary Dell Chilton (CIBA-GEIGY Biotechnology Research, Research Triangle Park, NC)

"Gene Transfer to Plants: The Cutting Edge" 


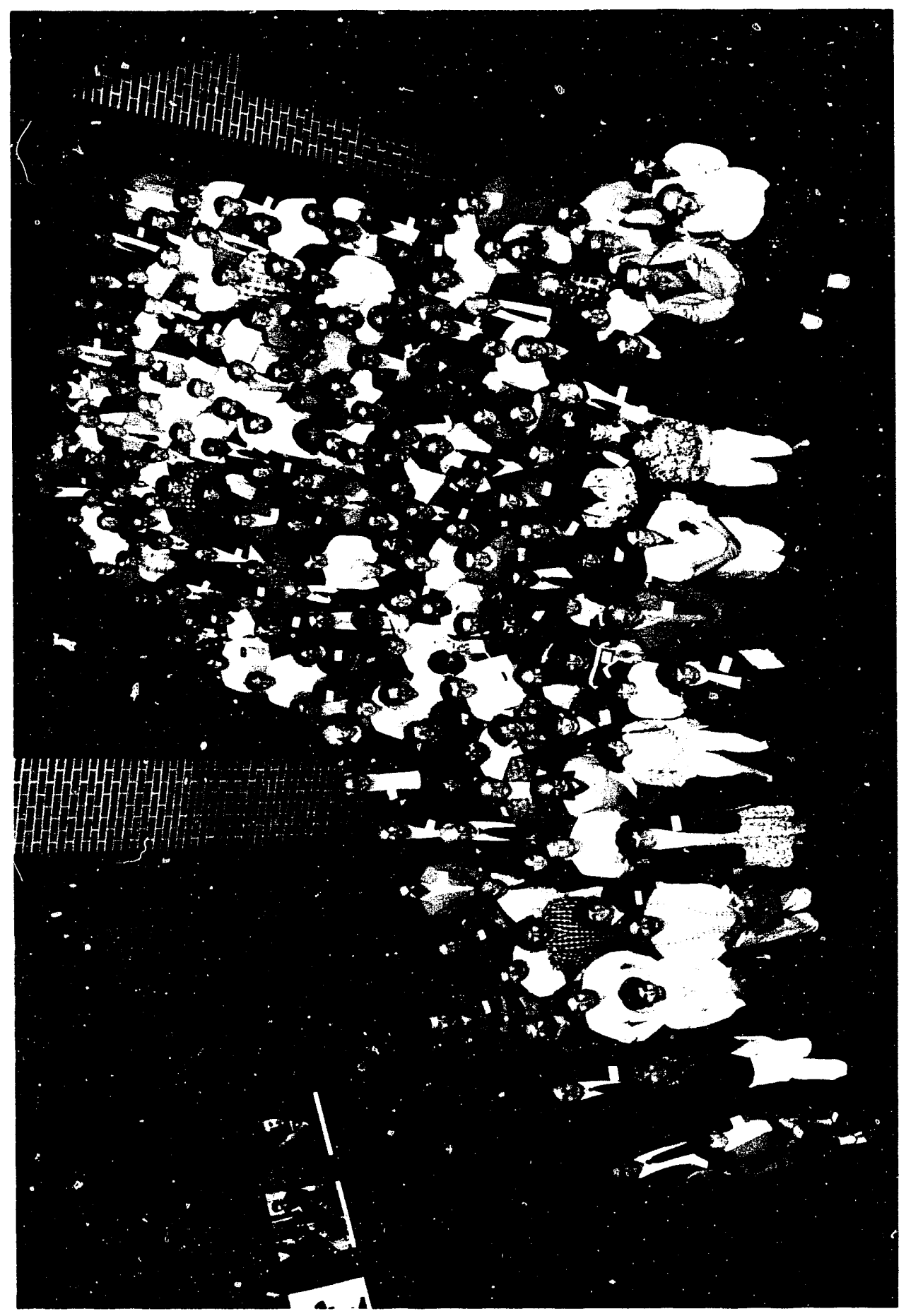




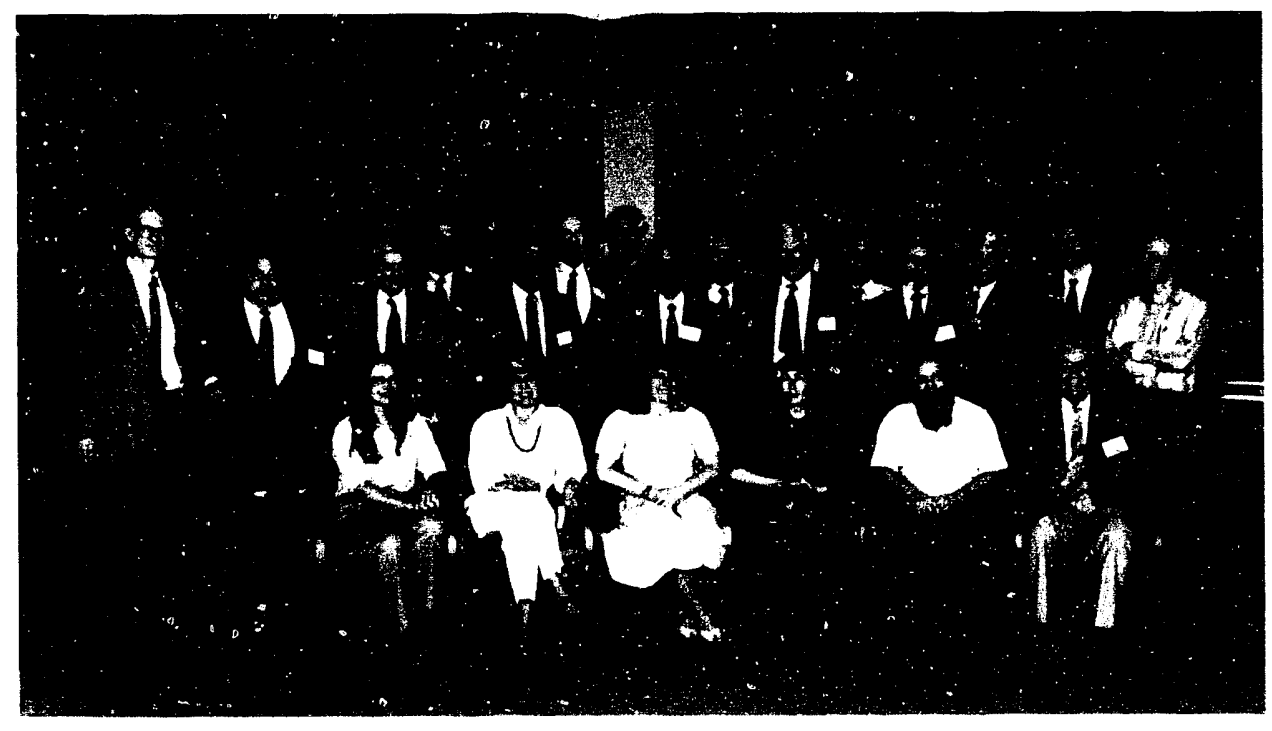

Current and former faculty members. Left to right, standing: C. Peter Wolk, Philip Filner, Joseph Varner, Kenneth Poff, John Scandalios, Jan A.D. Zeevaart, Lee Mclntosh, Hans Kende, Derek T.A. Lamport, Robert Rabson (DOE), Frans de Bruijn, Anton Lang, Andrew Hanson, Charles Arntzen, Jonathan Walton, Christopher Somerville; sitting, Shauna Somerville, Deborah Delmer, Pamela Green, Natasha Raikhel, Thomas Newman and Uoyd Wilson
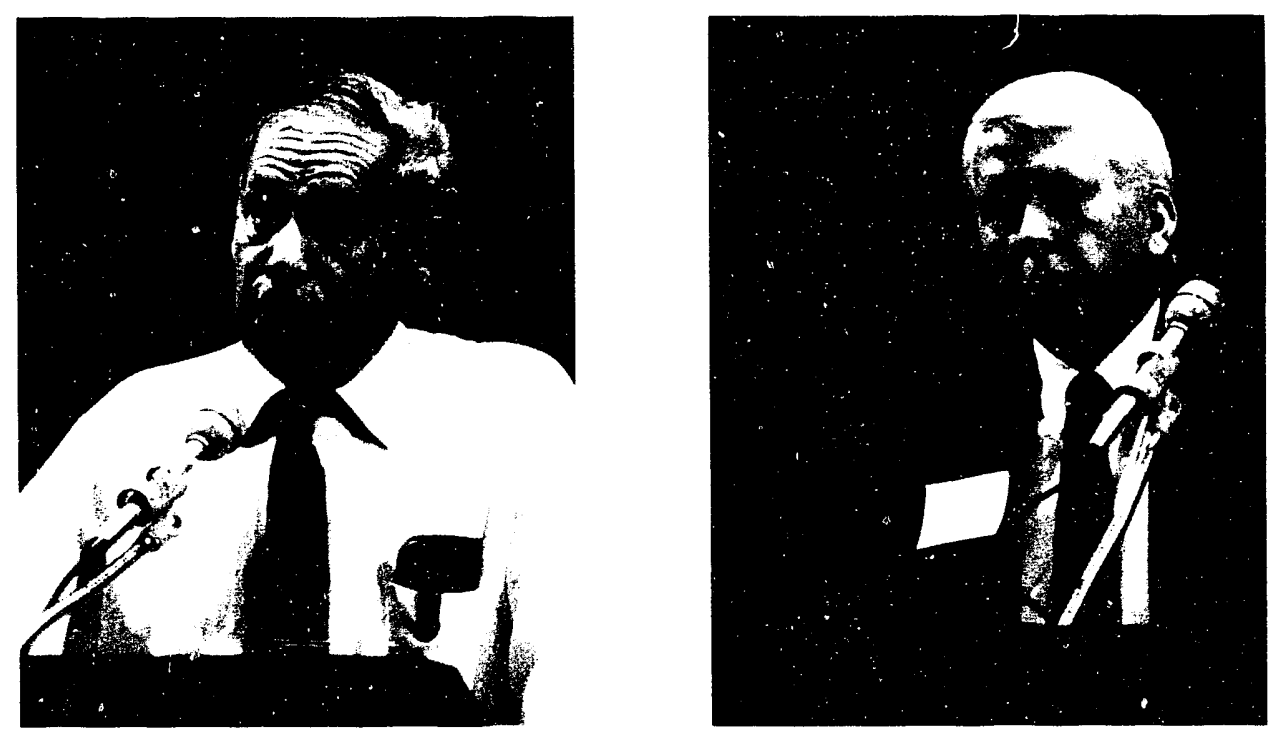

Anton Lang (1965 - 1978)

Former Directors

Charles Arntzen (1980 - 1984) 
Some of the Speakers at the Symposium

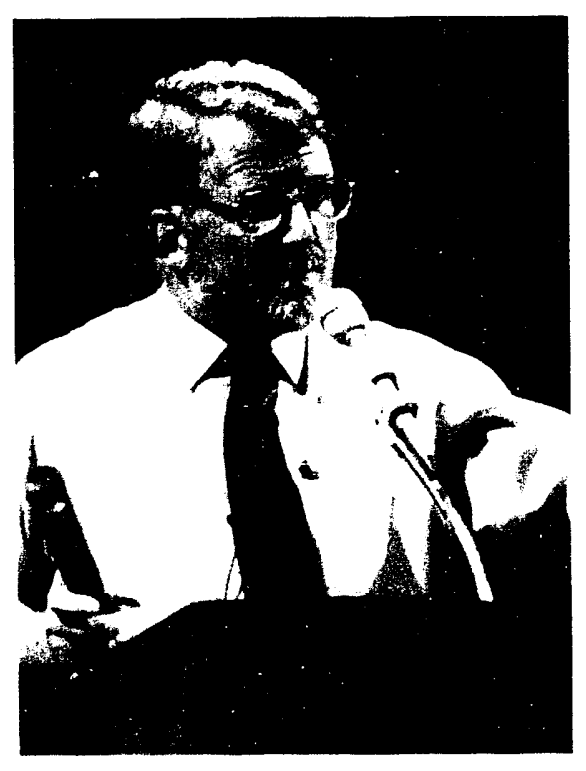

Jeff Schell

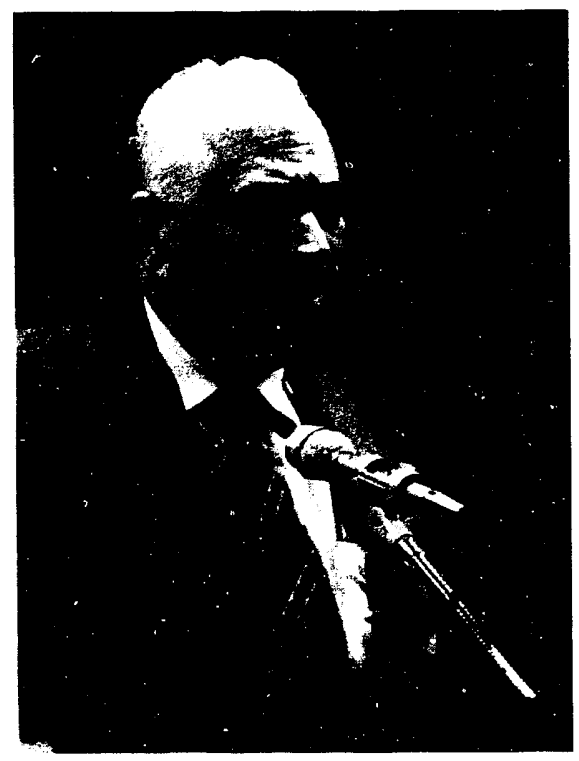

Robert Rabson

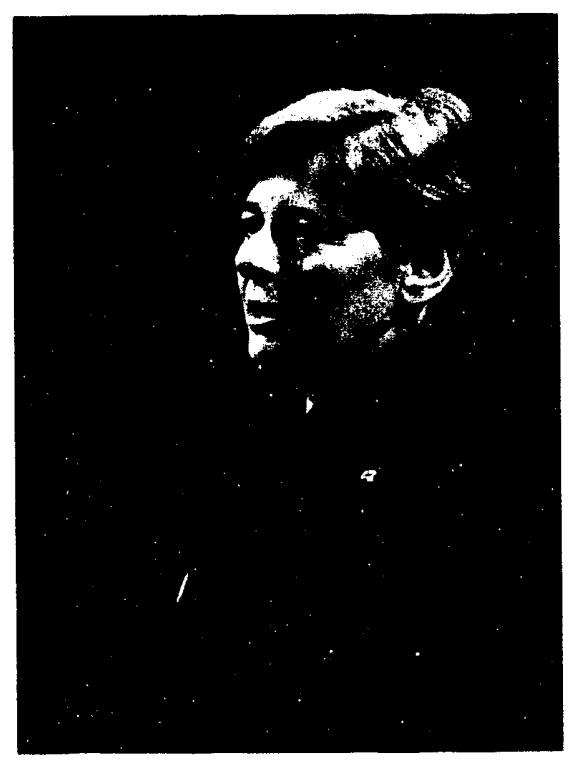

Adrienne Clarke

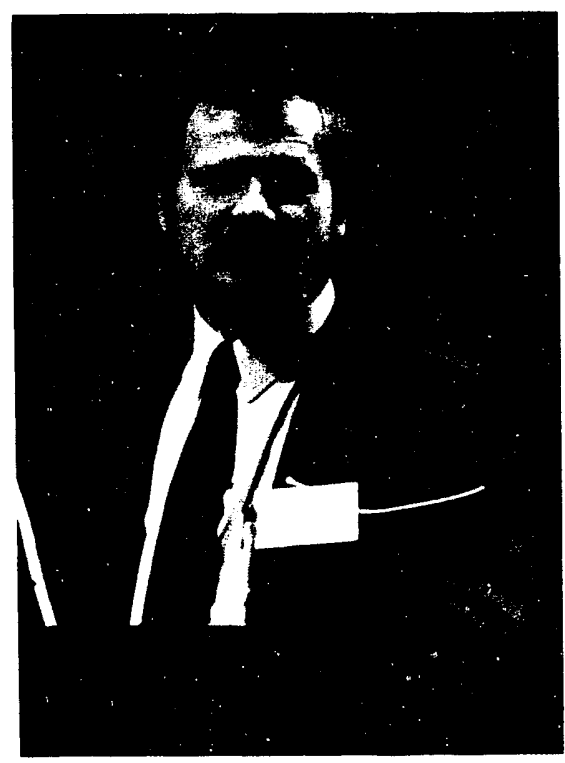

Hartmut Michel 

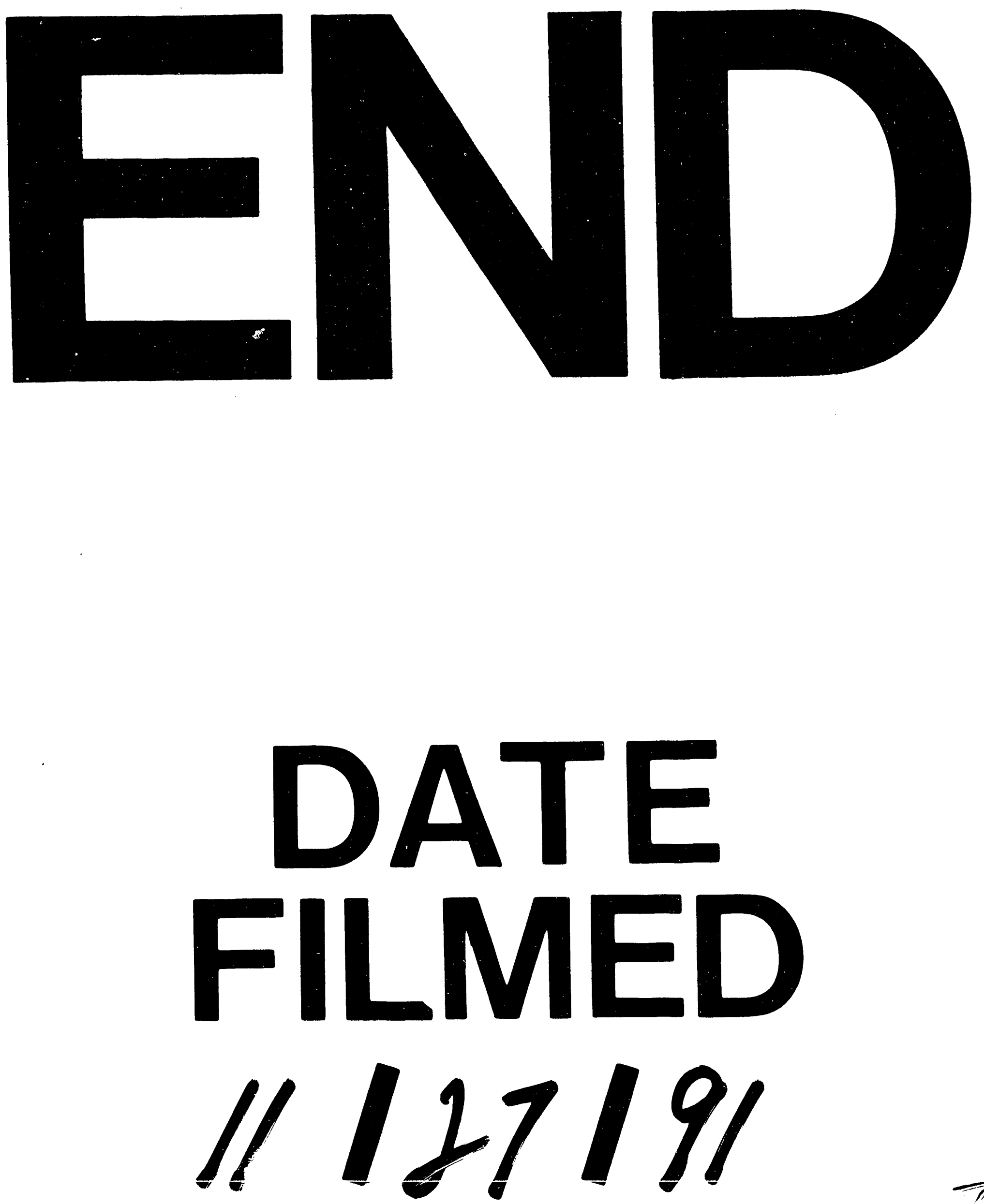
